\title{
A Concise Synthesis of (+)-SCH 351448
}

\section{Supporting Information}

\author{
Omid Soltani, Jef K. De Brabander* \\ Department of Biochemistry, The University of Texas Southwestern Medical Center at Dallas, 5323 \\ Harry Hines Boulevard, 75390-9038 \\ Email: jdebra@,biochem.swmed.edu
}

I. General Techniques: Unless otherwise noted, commercially available materials were used without further purification. All solvents were of HPLC or ACS grade. Solvents used for moisture sensitive operations were distilled from drying reagents under a nitrogen atmosphere: $\mathrm{Et}_{2} \mathrm{O}$ and $\mathrm{THF}$ from sodium benzophenone ketyl; benzene and toluene from sodium; $\mathrm{CH}_{2} \mathrm{Cl}_{2}$ from $\mathrm{CaH}_{2}$, pyridine over solid $\mathrm{KOH}$, anhydrous N,Ndimethylformamide, and $\mathrm{CH}_{3} \mathrm{CN}$ were purchased from commercial sources. Reactions were performed under an atmosphere of nitrogen with magnetic stirring unless noted otherwise. All photochemical reactions were performed with a Rayonett RPR-100 reactor fitted with a test tube carousel and $300 \mathrm{~nm}$ bulbs. Flash chromatography (FC) was performed using E Merck silica gel 60 (240-400 mesh) according to the protocol of Still, Kahn, and Mitra $(J$. Org. Chem. 1978, 43, 2923). Thin layer chromatography was performed performed using precoated plates purchased from $E$. Merck (silicagel $60 \mathrm{PF} 254,0.25 \mathrm{~mm}$ ) that were visualized using a $\mathrm{KMnO}_{4}$ or Ce (IV) stain.

Nuclear magnetic resonance (NMR) spectra were recorded on a Varian Inova-400 or Mercury-300 spectrometer at operating frequencies of $400 / 300 \mathrm{MHz}\left({ }^{1} \mathrm{H} \mathrm{NMR}\right)$ or $100 / 75 \mathrm{MHz}\left({ }^{13} \mathrm{C} \mathrm{NMR}\right)$. Chemical shifts $(\delta)$ are given in ppm relative to residual solvent (usually chloroform $\delta 7.26$ for ${ }^{1} \mathrm{H}$ NMR or $\delta 77.23$ for proton decoupled ${ }^{13} \mathrm{C}$ NMR), and coupling constants $(J)$ in Hz. Multiplicity is tabulated as s for singlet, $\mathrm{d}$ for doublet, $\mathrm{t}$ for triplet, $\mathrm{q}$ for quadruplet, and $\mathrm{m}$ for multiplet, whereby the prefix app is applied in cases where the true multiplicity is unresolved, and $b r$ when the signal in question is broadened.

Infrared spectra were recorded on a Perkin-ElmerI 1000 series FTIR with wavenumbers expressed in $\mathrm{cm}^{-1}$ using samples prepared as thin films between salt plates. Electrospray ionization mass spectra (ESI-MS) were recorded on a Shimadzu 2010-LCMS. Optical rotations were measured at $20{ }^{\circ} \mathrm{C}$ on a Rudolph Research Analytical Autopol ${ }^{\circledR}$ IV polarimeter.

\section{Experimental Procedures:}

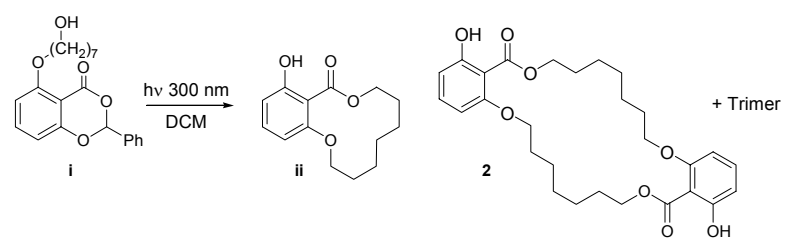

A solution of benzodioxinone $\mathbf{i}(150 \mathrm{mg}, 0.42 \mathrm{mmol})$ in freshly distilled $\mathrm{CH}_{2} \mathrm{Cl}_{2}(0.01 \mathrm{M})$ was transferred to an ovendried borosilicate test tube. The test tube was sealed with a rubber septum and parafilm and the solution was photolyzed at $300 \mathrm{~nm}$ for $4 \mathrm{~h}$. The solvent was removed and the crude material was purified by FC $(10 / 90 \mathrm{EtOAc} / \mathrm{Hex})$ to afford $20 \mathrm{mg}(19 \%)$ of lactone ii, $25 \mathrm{mg}(24 \%)$ of dimer 2 , and traces $(<1 \%)$ of trimer.

The same procedure at $0.14 \mathrm{M}$ concentration afford traces of macrocycle ii $(<1 \%), 14 \mathrm{mg}(14 \%)$ of dimer 2 and 7 $\mathrm{mg}(6 \%)$ of trimer.

Lactone ii: ${ }^{1} \mathrm{H}$ NMR $\left(400 \mathrm{MHz}, \mathrm{CDCl}_{3}\right) \delta 11.27(1 \mathrm{H}, \mathrm{s}), 7.28(1 \mathrm{H}, \mathrm{t}, J=8.0 \mathrm{~Hz}), 6.56(1 \mathrm{H}, \mathrm{d}, J=8.4 \mathrm{~Hz}), 6.39(1 \mathrm{H}$, $\mathrm{d}, J=8.4 \mathrm{~Hz}), 4.34(2 \mathrm{H}, \mathrm{t}, J=5.6 \mathrm{~Hz}), 4.01(2 \mathrm{H}, \mathrm{t}, J=5.6 \mathrm{~Hz}), 1.77-1.88(4 \mathrm{H}, \mathrm{m}), 1.56-1.69(6 \mathrm{H}, \mathrm{m})$.

Dimer 2: ${ }^{1} \mathrm{H}$ NMR $\left(400 \mathrm{MHz}, \mathrm{CDCl}_{3}\right) \delta 11.67(2 \mathrm{H}, \mathrm{s}), 7.30(2 \mathrm{H}, \mathrm{t}, J=8.0 \mathrm{~Hz}), 6.57(2 \mathrm{H}, \mathrm{d}, J=8.4 \mathrm{~Hz}), 6.39(2 \mathrm{H}$, $\mathrm{d}, J=8.8 \mathrm{~Hz}), 4.38(4 \mathrm{H}, \mathrm{t}, J=6.0 \mathrm{~Hz}), 4.00(4 \mathrm{H}, \mathrm{t}, J=5.6 \mathrm{~Hz}), 1.76-1.85(8 \mathrm{H}, \mathrm{m}), 1.50-1.60(8 \mathrm{H}, \mathrm{m}), 1.42-1.49$ (4H, m). MS (ES) $m / z(\%): 523.25$ ([MNa $\left.]^{+}, 100\right)$. 
Trimer: ${ }^{1} \mathrm{H}$ NMR $\left(400 \mathrm{MHz}, \mathrm{CDCl}_{3}\right) \delta 11.67(3 \mathrm{H}, \mathrm{s}), 7.28(3 \mathrm{H}, \mathrm{t}, J=8.0 \mathrm{~Hz}), 6.55(3 \mathrm{H}, \mathrm{d}, J=8.4 \mathrm{~Hz}), 6.37(3 \mathrm{H}, \mathrm{d}$, $J=8.4 \mathrm{~Hz}), 4.37(6 \mathrm{H}, \mathrm{t}, J=6.0 \mathrm{~Hz}), 3.98(6 \mathrm{H}, \mathrm{t}, J=5.8 \mathrm{~Hz}), 1.75-1.85(12 \mathrm{H}, \mathrm{m}), 1.50-1.60(12 \mathrm{H}, \mathrm{m}), 1.41-1.48$ $(6 \mathrm{H}, \mathrm{m}) . \mathrm{MS}(\mathrm{ES}) \mathrm{m} / z(\%): 773.20\left([\mathrm{MNa}]^{+}, 100\right)$.

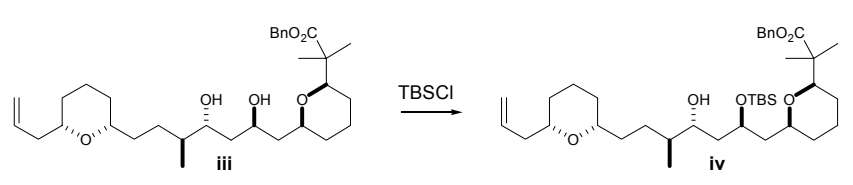

To a stirred solution of diol iii $^{1}(1.11 \mathrm{~g}, 2.10 \mathrm{mmol})$, imidazole (873 $\mathrm{mg}, 12.84 \mathrm{mmol}$ ), and DMAP (26 mg, $0.214 \mathrm{mmol})$ in anhydrous $\mathrm{DMF}(15 \mathrm{~mL})$ at $0{ }^{\circ} \mathrm{C}$ was added TBSCl (969 $\mathrm{mg}, 6.43 \mathrm{mmol}$ ). The reaction was allowed to stir for $2 \mathrm{~h}$ at $0{ }^{\circ} \mathrm{C}$ before addition of water $(150$

$\mathrm{mL})$. The solution was extracted with $\mathrm{Et}_{2} \mathrm{O}(3 \times 200 \mathrm{~mL})$, dried over $\mathrm{MgSO}_{4}$, concentrated, and purified by $\mathrm{FC}$ $\left(5 / 95\right.$, EtOAC/Hex) to afford $1.10 \mathrm{~g}(80 \%)$ of iv. $[\alpha]_{\mathrm{D}}=-11.0\left(\mathrm{CHCl}_{3}, c 0.15\right)$. IR (film) $3511,2935,2858,1737$, $1462,1389,1258,1087,1051,836,776 \mathrm{~cm}^{-1} ;{ }^{1} \mathrm{H}$ NMR $\left(400 \mathrm{MHz}, \mathrm{CDCl}_{3}\right) \delta 7.26-7.38(5 \mathrm{H}, \mathrm{m}), 5.84(1 \mathrm{H}, \mathrm{dddd}, J$ $=6.8,6.8,10.0,16.4 \mathrm{~Hz}), 5.14(1 \mathrm{H}, \mathrm{d}, J=12.8 \mathrm{~Hz}), 5.06(1 \mathrm{H}, \mathrm{d}, J=12.8 \mathrm{~Hz}), 5.03(1 \mathrm{H}$, app d, $J=6.8 \mathrm{~Hz}), 5.00$ $(1 \mathrm{H}$, app d, $J=16.4 \mathrm{~Hz}), 4.18-4.26(1 \mathrm{H}, \mathrm{m}), 3.68-3.76(1 \mathrm{H}, \mathrm{m}), 3.50(1 \mathrm{H}, \mathrm{d}, J=10.8 \mathrm{~Hz}), 3.19-3.34(3 \mathrm{H}, \mathrm{m})$, 2.26-2.36 (1H, m), 2.09-2.18 (1H, m), 1.77-1.88 (2H, m), $1.71(2 \mathrm{H}, \mathrm{t}, J=6.8 \mathrm{~Hz}), 1.39-1.63(12 \mathrm{H}, \mathrm{m}), 1.08-1.29$ $(5 \mathrm{H}, \mathrm{m}), 1.20(3 \mathrm{H}, \mathrm{s}), 1.12(3 \mathrm{H}, \mathrm{s}), 0.88(9 \mathrm{H}, \mathrm{s}), 0.84(3 \mathrm{H}, \mathrm{d}, J=6.8 \mathrm{~Hz}), 0.07(3 \mathrm{H}, \mathrm{s}), 0.06(3 \mathrm{H}, \mathrm{s}) ;{ }^{13} \mathrm{C}$ NMR $(75$ $\left.\mathrm{MHz}, \mathrm{CDCl}_{3}\right) \delta 176.8,136.5,135.6,128.7,128.2,127.8,116.4,82.2,78.6,77.6,75.3,72.0,68.7,66.3,46.9,42.6$, $41.3,39.1,36.3,34.3,32.3,31.6,31.5,28.2,26.0,25.2,23.8,21.4,20.5,18.1,15.2,-4.5,-4.8$. MS (ES) $\mathrm{m} / z(\%)$ : $667.52\left([\mathrm{MNa}]^{+}, 100\right)$.

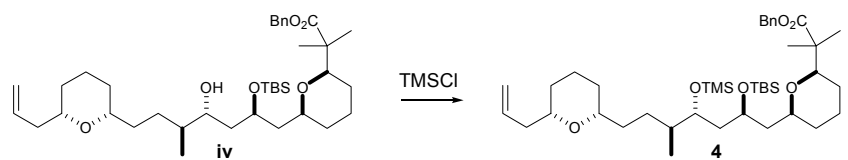

Compound 4. To a stirred solution of alcohol iv (1.3 g, $2.05 \mathrm{mmol})$ and $\mathrm{Et}_{3} \mathrm{~N}(5.6 \mathrm{~mL}, 41.0 \mathrm{mmol})$, in $\mathrm{CH}_{2} \mathrm{Cl}_{2}(15$ $\mathrm{mL})$ at $0{ }^{\circ} \mathrm{C}$ was added TMSCl $(2.6 \mathrm{~mL}, 20.5 \mathrm{mmol})$. The reaction was brought to ambient temperature and stirred for $12 \mathrm{~h}$. The reaction solution was poured into water (100 $\mathrm{mL})$ and extracted with $\mathrm{Et}_{2} \mathrm{O}(3 \times 150 \mathrm{~mL})$. The organic phase was dried over $\mathrm{MgSO}_{4}$, concentrated, and purified by FC $\left(5 / 95\right.$, EtOAc/Hex) to afford $1.2 \mathrm{~g}(84 \%)$ of $4 .[\alpha]_{\mathrm{D}}=+11.5\left(\mathrm{CHCl}_{3}, c 1.0\right)$. IR (film) 2936, 2858, 1738, 1472, 1388, 1251, 1086, 838, $774 \mathrm{~cm}^{-1}$; ${ }^{1} \mathrm{H}$ NMR $\left(400 \mathrm{MHz}, \mathrm{CDCl}_{3}\right) \delta 7.19-7.30(5 \mathrm{H}, \mathrm{m}), 5.77(1 \mathrm{H}$, dddd, $J=7.2$, $7.2,10.4,17.2 \mathrm{~Hz}), 5.06(1 \mathrm{H}, \mathrm{d}, J=12.8 \mathrm{~Hz}), 5.02(1 \mathrm{H}, \mathrm{d}, J=12.8 \mathrm{~Hz}), 4.97(1 \mathrm{H}$, app d, $J=17.2 \mathrm{~Hz}), 4.92(1 \mathrm{H}$, app d, $J=9.6 \mathrm{~Hz}), 3.82-3.90(1 \mathrm{H}, \mathrm{m}), 3.70-3.74(1 \mathrm{H}, \mathrm{m}), 3.37(1 \mathrm{H}, \mathrm{d}, J=10.0 \mathrm{~Hz}), 3.27-3.32(1 \mathrm{H}, \mathrm{m}), 3.16-3.24$ $(1 \mathrm{H}, \mathrm{m}), 3.09-3.15(1 \mathrm{H}, \mathrm{m}), 2.19-2.28(1 \mathrm{H}, \mathrm{m}), 2.05-2.10(1 \mathrm{H}, \mathrm{m}), 1.60-1.77(3 \mathrm{H}, \mathrm{m}), 1.46-1.53(3 \mathrm{H}, \mathrm{m}), 1.23-$ $1.43(9 \mathrm{H}, \mathrm{m}), 1.17(3 \mathrm{H}, \mathrm{s}), 1.07(3 \mathrm{H}, \mathrm{s}), 0.94-1.20(6 \mathrm{H}, \mathrm{m}), 0.81(9 \mathrm{H}, \mathrm{s}), 0.75(3 \mathrm{H}, \mathrm{d}, J=6.4 \mathrm{~Hz}), 0.01(9 \mathrm{H}, \mathrm{s})$, $-0.01(3 \mathrm{H}, \mathrm{s}),-0.02(3 \mathrm{H}, \mathrm{s}) ;{ }^{13} \mathrm{C} \mathrm{NMR}\left(75 \mathrm{MHz}, \mathrm{CDCl}_{3}\right) \delta 176.7,136.6,135.6,128.6,128.1,128.0,116.4,81.9$, 78.5, 77.7, 75.2, 73.5, 67.3, 66.2, 47.0, 45.9, 41.3, 40.2, 39.4, 34.9, 32.2, 31.7, 31.4, 29.1, 26.2, 25.9, 23.9, 23.0, 19.9, 18.3, 14.1, 1.1, -3.4, -3.9. MS (ES) $m / z(\%): 739.50\left([\mathrm{MNa}]^{+}, 5\right), 645.45\left(\left[\mathrm{MH}_{2}-\mathrm{TMS}\right]^{+}, 100\right)$.

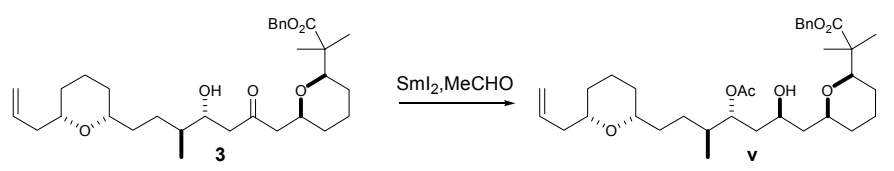

Evans-Tishchenko reduction of hydroxy ketone 3. To a stirred solution of hydroxy ketone $\mathbf{3}^{1}(1.95 \mathrm{~g}, 3.7$ $\mathrm{mmol})$ and acetaldehyde $(1.59 \mathrm{~mL}, 28.4 \mathrm{mmol})$ in THF $(20 \mathrm{~mL})$ at $-10{ }^{\circ} \mathrm{C}$ was added a freshly prepared solution of samarium iodide $(0.1 \mathrm{M}$ in THF, $11 \mathrm{~mL}, 1.1 \mathrm{mmol})$ dropwise. The reaction was allowed to warm to $0{ }^{\circ} \mathrm{C}$ and stirred at that temperature for $8 \mathrm{~h}$. Saturated aq $\mathrm{NH}_{4} \mathrm{Cl}(5 \mathrm{~mL})$ was added, followed by water $(50 \mathrm{~mL})$ and extraction with $\mathrm{Et}_{2} \mathrm{O}(3 \times 100 \mathrm{~mL})$. The organic phase was dried over $\mathrm{MgSO}_{4}$, concentrated, and purified by $\mathrm{FC}$ $(1 / 4$, EtOAc/Hex $)$ to afford $1.73 \mathrm{~g}(82 \%)$ of $\mathbf{v} .[\alpha]_{\mathrm{D}}=+6.5\left(\mathrm{CHCl}_{3}, c\right.$ 3.0). IR (film) 3527, 2936, 2860, 1732, 1373, 1247, 1085, $912 \mathrm{~cm}^{-1} ;{ }^{1} \mathrm{H}$ NMR $\left(400 \mathrm{MHz}, \mathrm{CDCl}_{3}\right) \delta 7.26-7.38(5 \mathrm{H}, \mathrm{m}), 5.83(1 \mathrm{H}$, dddd, $J=7.6,7.6,10.4,17.2$ $\mathrm{Hz}), 5.15(1 \mathrm{H}, \mathrm{d}, J=12.4 \mathrm{~Hz}), 5.10(1 \mathrm{H}, \mathrm{d}, J=12.8 \mathrm{~Hz}), 4.89-5.09(3 \mathrm{H}, \mathrm{m}), 3.66-3.74(1 \mathrm{H}, \mathrm{m}), 3.57(1 \mathrm{H}, \mathrm{d}, J=$ $10.8 \mathrm{~Hz}), 3.49-3.56(1 \mathrm{H}, \mathrm{m}), 3.47(1 \mathrm{H}, \mathrm{s}), 3.25-3.32(1 \mathrm{H}, \mathrm{m}), 3.15-3.24(1 \mathrm{H}, \mathrm{m}), 2.23-2.34(1 \mathrm{H}, \mathrm{m}), 2.08-2.18$ $(1 \mathrm{H}, \mathrm{m}), 2.01(3 \mathrm{H}, \mathrm{s}), 1.76-1.87(2 \mathrm{H}, \mathrm{m}), 1.40-1.73(14 \mathrm{H}, \mathrm{m}), 1.02-1.33(5 \mathrm{H}, \mathrm{m}), 1.19(3 \mathrm{H}, \mathrm{s}), 1.14(3 \mathrm{H}, \mathrm{s}), 0.86$

\footnotetext{
${ }^{1}$ Bhattacharjee, A.; Soltani, O.; De Brabander, J. K. Org.Lett. 2002, 4, 481-484.
} 
$(3 \mathrm{H}, \mathrm{d}, J=6.8 \mathrm{~Hz}) ;{ }^{13} \mathrm{C} \mathrm{NMR}\left(75 \mathrm{MHz}, \mathrm{CDCl}_{3}\right) \delta 176.7,171.3,136.5,135.5,128.6,128.1,116.4,82.8,78.8,78.2$, 77.5, 75.0, 67.4, 66.5, 46.8, 43.5, 41.2, 38.4, 36.9, 34.2, 32.0, 31.6, 31.4, 28.3, 25.0, 23.8, 23.5, 21.5, 21.3, 20.3, 15.1. MS (ES) $m / z(\%): 595.40\left([\mathrm{MNa}]^{+}, 100\right)$.

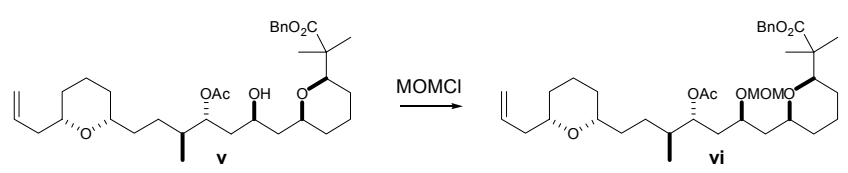

MOM ether vi. To a stirred solution of free alcohol $\mathbf{v}$ (1.67 g, $2.92 \mathrm{mmol})$, and ${ }^{i} \operatorname{Pr}_{2} \mathrm{NEt}(7.60 \mathrm{~mL}, 43.8 \mathrm{mmol})$ in $\mathrm{CH}_{2} \mathrm{Cl}_{2}(25 \mathrm{~mL})$ at ambient temperature was added $\mathrm{MOMCl}(1.55 \mathrm{~mL}, 20.4 \mathrm{mmol})$. The reaction was stirred

for $12 \mathrm{~h}$ at ambient temperature and $3 \mathrm{~h}$ at $50{ }^{\circ} \mathrm{C}$. The reaction solution was poured into water $(50 \mathrm{~mL})$ and extracted with $\mathrm{Et}_{2} \mathrm{O}(3 \times 100 \mathrm{~mL})$. The combined organic phase was dried over $\mathrm{MgSO}_{4}$, concentrated, and purified by FC $(1 / 9, \mathrm{EtOAc} / \mathrm{Hex})$ to afford $1.71 \mathrm{~g}(95 \%)$ of vi. $[\alpha]_{\mathrm{D}}=-0.8\left(\mathrm{CHCl}_{3}, c 1.0\right)$. IR (film) $2935,1732,1455,1372$, $1243,1087,1041,918 \mathrm{~cm}^{-1} ;{ }^{1} \mathrm{H}$ NMR $\left(400 \mathrm{MHz}, \mathrm{CDCl}_{3}\right) \delta 7.28-7.38(5 \mathrm{H}, \mathrm{m}), 5.83(1 \mathrm{H}, \mathrm{dddd}, J=7.2,7.2,10.4$, $17.2 \mathrm{~Hz}), 5.12(2 \mathrm{H}, \mathrm{s}), 5.06-5.08(1 \mathrm{H}, \mathrm{m}), 4.97-5.04(2 \mathrm{H}, \mathrm{m}), 4.61(1 \mathrm{H}, \mathrm{d}, J=6.8 \mathrm{~Hz}), 4.53(1 \mathrm{H}, \mathrm{d}, J=6.8 \mathrm{~Hz})$, $3.62-3.71(1 \mathrm{H}, \mathrm{m}), 3.47(1 \mathrm{H}, \mathrm{dd}, J=1.6,11.2 \mathrm{~Hz}), 3.17-3.42(3 \mathrm{H}, \mathrm{m}), 3.34(3 \mathrm{H}, \mathrm{s}), 2.25-2.34(1 \mathrm{H}, \mathrm{m}), 2.09-2.18$ $(1 \mathrm{H}, \mathrm{m}), 2.01(3 \mathrm{H}, \mathrm{s}), 1.76-1.89(2 \mathrm{H}, \mathrm{m}), 1.68-1.76(1 \mathrm{H}, \mathrm{m}), 1.36-1.62(12 \mathrm{H}, \mathrm{m}), 1.02-1.31(6 \mathrm{H}, \mathrm{m}), 1.23(3 \mathrm{H}, \mathrm{s})$, $1.15(3 \mathrm{H}, \mathrm{s}), 0.85(3 \mathrm{H}, \mathrm{d}, J=6.4 \mathrm{~Hz}) ;{ }^{13} \mathrm{C} \mathrm{NMR}\left(75 \mathrm{MHz}, \mathrm{CDCl}_{3}\right) \delta 176.4,170.6,136.4,135.4,128.5,127.9$, 127.8, 116.2, 96.3, 82.1, 78.0, 77.3, 74.8, 74.3, 72.2, 66.0, 55.8, 46.7, 42.1, 41.0, 36.6, 35.0, 34.1, 31.9, 31.5, 31.2, 28.4, 25.5, 23.6, 21.8, 21.2, 20.4, 14.7. MS (ES) $m / z(\%): 639.35\left([\mathrm{MNa}]^{+}, 100\right)$.

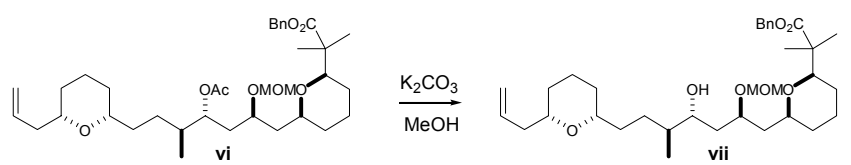

Acetate hydrolysis. To a stirred solution of acetate vi (400 $\mathrm{mg}, 0.65 \mathrm{mmol})$ in anhydrous methanol $(7 \mathrm{~mL})$ was added anhydrous $\mathrm{K}_{2} \mathrm{CO}_{3}(358 \mathrm{mg}, 2.59 \mathrm{mmol})$. The reaction was allowed to stir for $48 \mathrm{~h}$ at ambient temperature before additional $\mathrm{K}_{2} \mathrm{CO}_{3}(1.0 \mathrm{~g}, 7.24 \mathrm{mmol})$ was added. After $60 \mathrm{~h}$ total reaction time, the reaction was poured into water $(100 \mathrm{~mL})$ and extracted with $\mathrm{Et}_{2} \mathrm{O}(3 \times 50 \mathrm{~mL})$. The organic phase was dried over $\mathrm{MgSO}_{4}$, concentrated, and purified by $\mathrm{FC}(15 / 85, \mathrm{EtOAc} / \mathrm{Hex})$ to afford $261 \mathrm{mg}(70 \%)$ alcohol vii. $[\alpha]_{\mathrm{D}}=-15.8\left(\mathrm{CHCl}_{3}, c 0.7\right)$. IR (film) $3512,2934,1730,1457,1374,1268,1149,1088,1039,914 \mathrm{~cm}^{-1} ;{ }^{1} \mathrm{H}$ NMR $\left(400 \mathrm{MHz}, \mathrm{CDCl}_{3}\right) \delta 7.22-7.40(5 \mathrm{H}$, $\mathrm{m}), 5.84(1 \mathrm{H}$, dddd, $J=6.8,6.8,9.6,16.4 \mathrm{~Hz}), 5.13(2 \mathrm{H}, \mathrm{s}), 5.05(1 \mathrm{H}, \mathrm{d}, J=17.6 \mathrm{~Hz}), 5.01(1 \mathrm{H}, \mathrm{d}, J=10.4 \mathrm{~Hz})$, $4.63(1 \mathrm{H}, \mathrm{d}, J=6.8 \mathrm{~Hz}), 4.59(1 \mathrm{H}, \mathrm{d}, J=6.8 \mathrm{~Hz}), 3.92-4.00(1 \mathrm{H}, \mathrm{m}), 3.64-3.71(1 \mathrm{H}, \mathrm{m}), 3.52(1 \mathrm{H}, \mathrm{d}, J=10.8 \mathrm{~Hz})$, 3.19-3.42 $(3 \mathrm{H}, \mathrm{m}), 3.37(3 \mathrm{H}, \mathrm{s}), 2.95(1 \mathrm{H}, \mathrm{br} \mathrm{s}), 2.27-2.35(1 \mathrm{H}, \mathrm{m}), 2.09-2.18(1 \mathrm{H}, \mathrm{m}), 1.77-1.89(2 \mathrm{H}, \mathrm{m}), 1.41-$ $1.68(12 \mathrm{H}, \mathrm{m}), 1.12-1.29(7 \mathrm{H}, \mathrm{m}), 1.21(3 \mathrm{H}, \mathrm{s}), 1.13(3 \mathrm{H}, \mathrm{s}), 0.88(3 \mathrm{H}, \mathrm{d}, J=7.2 \mathrm{~Hz}) ;{ }^{13} \mathrm{C}$ NMR $\left(75 \mathrm{MHz}, \mathrm{CDCl}_{3}\right)$ $\delta 176.8,136.5,135.4,128.5,128.0,127.8,116.3,96.1,82.3,78.5,77.5,75.0,73.5,71.6,66.2,55.8,46.8,41.2$, $41.1,39.0,37.1,34.2,32.0,31.5,31.3,28.2,25.2,23.7,21.4,20.5,15.3$. MS (ES) $m / z(\%): 597.45$ ([MNa] $\left.]^{+}, 100\right)$.

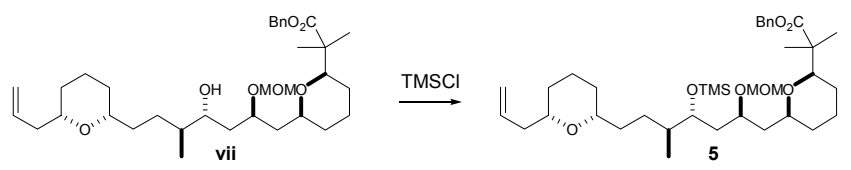

Compound 5. To a stirred solution of alcohol vii (140 mg, $0.244 \mathrm{mmol})$ in $\mathrm{CH}_{2} \mathrm{Cl}_{2}(5 \mathrm{~mL})$ at $0{ }^{\circ} \mathrm{C}$ was added $\mathrm{Et}_{3} \mathrm{~N}$ $(0.68 \mathrm{~mL}, 4.88 \mathrm{mmol})$ followed by TMSCl $(0.31 \mathrm{~mL}, 2.44$ $\mathrm{mmol})$. After $1 \mathrm{~h}$, the reaction was quenched by addition of water $(30 \mathrm{~mL})$ and extracted with $\mathrm{Et}_{2} \mathrm{O}(2 \times 50 \mathrm{~mL})$. The organic phase was then dried over $\mathrm{MgSO}_{4}$, concentrated, and purified by FC $\left(1 / 9\right.$, EtOAc/Hex) to afford $150 \mathrm{mg}(96 \%)$ of silyl ether 5. $[\alpha]_{\mathrm{D}}=-2.8\left(\mathrm{CHCl}_{3}, c 0.3\right)$. IR (film) 2936, 1736, 1456, 1375, 1250, 1087, 1042, 918, $840 \mathrm{~cm}^{-1} ;{ }^{1} \mathrm{H}$ NMR (400 MHz, $\left.\mathrm{CDCl}_{3}\right) \delta 7.29-7.38(5 \mathrm{H}, \mathrm{m}), 5.83$ $(1 \mathrm{H}$, dddd, $J=6.4,6.4,10.4,16.8 \mathrm{~Hz}), 5.11(2 \mathrm{H}, \mathrm{s}), 5.05(1 \mathrm{H}$, app d, $J=17.2 \mathrm{~Hz}), 5.00(1 \mathrm{H}$, app d, $J=10.0 \mathrm{~Hz})$, $4.63(2 \mathrm{H}, \mathrm{s}), 3.70-3.84(2 \mathrm{H}, \mathrm{m}), 3.46(1 \mathrm{H}, \mathrm{d}, J=7.2 \mathrm{~Hz}), 3.38-3.44(1 \mathrm{H}, \mathrm{m}), 3.36(3 \mathrm{H}, \mathrm{s}), 3.25-3.34(1 \mathrm{H}, \mathrm{m})$, 3.16-3.24 (1H, m), 2.26-2.35 (1H, m), 2.09-2.18 (1H, m), 1.77-1.86 (3H, m), 1.30-1.62 (12H, m), 0.94-1.29 $(6 \mathrm{H}$, s), $1.23(3 \mathrm{H}, \mathrm{s}), 1.14(3 \mathrm{H}, \mathrm{s}), 0.84(3 \mathrm{H}, \mathrm{d}, J=6.8 \mathrm{~Hz}), 0.09(9 \mathrm{H}, \mathrm{s}) ;{ }^{13} \mathrm{C}$ NMR $\left(75 \mathrm{MHz}, \mathrm{CDCl}_{3}\right) \delta 176.5,136.6$, $135.4,128.5,127.9,127.8,116.3,96.3,82.0,78.3,77.5,75.0,73.7,73.0,66.0,55.6,46.8,42.7,41.1,39.5,37.7$, $34.8,31.9,31.6,31.3,29.0,25.7,23.7,22.3,20.2,13.8,0.76$. MS (ES) $m / z(\%): 669.40\left([\mathrm{MNa}]^{+}, 100\right)$. 

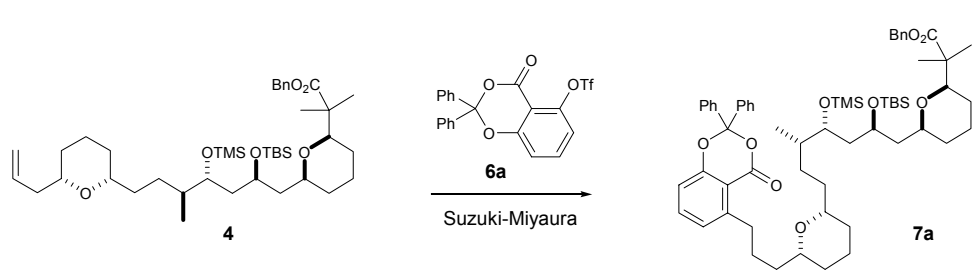

Compound 7a. To a roundbottom flask charged with bis-silyl ether 4 (292 $\mathrm{mg}, 0.408 \mathrm{mmol})$ and solid 9-BBN (100 mg, $0.816 \mathrm{mmol})$ was added freshly distilled and degassed THF $(2 \mathrm{~mL})$. The reaction was allowed to stir at ambient temperature for $17 \mathrm{~h}$. To this solution was added aqueous $3 \quad \mathrm{M} \mathrm{K}_{3} \mathrm{PO}_{4}(340 \mu \mathrm{L}, 1.02 \quad \mathrm{mmol})$, followed by a solution of $\mathbf{6 a}^{2}(275 \mathrm{mg}, 0.612 \mathrm{mmol})$ and $\mathrm{PdCl}_{2} \mathrm{dppf}(16.6 \mathrm{mg}, 0.204 \mathrm{mmol})$ in degassed DMF (2 $\mathrm{mL})$. After $3 \mathrm{~h}$ the reaction was poured into water $(30 \mathrm{~mL})$ and extracted with $\mathrm{Et}_{2} \mathrm{O}(3 \times 50 \mathrm{~mL})$. The combined organic phase was dried over $\mathrm{MgSO}_{4}$, filtered, concentrated, and purified by FC $(5 / 95 \rightarrow 15 / 85 \mathrm{EtOAc} / \mathrm{Hex})$ rapidly (to avoid silyl deprotection) to afford $363 \mathrm{mg}(87 \%)$ of $7 \mathbf{a}$. $[\alpha]_{\mathrm{D}}=+9.2\left(\mathrm{CHCl}_{3}, c 1.0\right)$. IR (film) 2934, 2857, 1742, $1607,1580,1266,1206,1091,837,775,757 \mathrm{~cm}^{-1} ;{ }^{1} \mathrm{H}$ NMR (400 MHz, $\left.\mathrm{CDCl}_{3}\right) \delta 7.47-7.51(4 \mathrm{H}, \mathrm{m}), 7.20-7.30$ $(12 \mathrm{H}, \mathrm{m}), 6.90(1 \mathrm{H}, \mathrm{d}, J=8.4 \mathrm{~Hz}), 6.74(1 \mathrm{H}, \mathrm{d}, J=8.0 \mathrm{~Hz}), 5.06(1 \mathrm{H}, \mathrm{d}, J=12.4 \mathrm{~Hz}), 5.02(1 \mathrm{H}, \mathrm{d}, J=12.4 \mathrm{~Hz})$, 3.83-3.86 (1H, m), 3.68-3.72 (1H, m), $3.38(1 \mathrm{H}, \mathrm{d}, J=10.8 \mathrm{~Hz}), 3.24-3.32(1 \mathrm{H}, \mathrm{m}), 3.04-3.13(2 \mathrm{H}, \mathrm{m}), 2.93-3.01$ $(1 \mathrm{H}, \mathrm{m}), 2.83-2.90(1 \mathrm{H}, \mathrm{m}), 1.77-1.82(2 \mathrm{H}, \mathrm{m}), 1.68-1.75(2 \mathrm{H}, \mathrm{m}), 1.44-1.54(4 \mathrm{H}, \mathrm{m}), 1.23-1.42(13 \mathrm{H}, \mathrm{m}), 1.17$ $(3 \mathrm{H}, \mathrm{s}), 1.07(3 \mathrm{H}, \mathrm{s}), 0.94-1.06(4 \mathrm{H}, \mathrm{m}), 0.78(9 \mathrm{H}, \mathrm{s}), 0.75(3 \mathrm{H}, \mathrm{d}, J=6.8 \mathrm{~Hz}), 0.01(9 \mathrm{H}, \mathrm{s}),-0.03(6 \mathrm{H}, \mathrm{s}) ;{ }^{13} \mathrm{C}$ NMR $\left(75 \mathrm{MHz}, \mathrm{CDCl}_{3}\right) \delta 176.8,160.4,157.7,148.6,140.0,136.6,135.4,129.3,129.2,128.7,128.1,128.0,126.8$, 126.7, 125.7, 115.6, 113.6, 106.0, 81.9, 78.3, 77.9, 75.2, 73.4, 67.3, 66.2, 47.0, 45.9, 40.3, 39.4, 36.3, 34.9, 34.4, $32.2,31.8,31.7,29.0,26.9,26.2,26.0,24.0,23.9,23.0,19.9,18.3,14.2,1.1,-3.4,-3.9$. MS (ES) $\mathrm{m} / z(\%): 969.65$ ([MHNa-TMS $\left.]^{+}, 90\right)$.

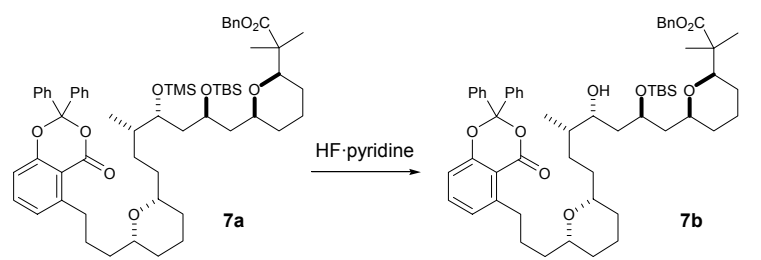

Compound 7b. To a stirred solution of bis-silyl ether 7a (322 $\mathrm{mg}, 0.316 \mathrm{mmol})$ in THF $(5 \mathrm{~mL})$ was added a solution of HF.pyridine $[1 \mathrm{~mL}$ from a stock solution composed of $70 \%$ HF.pyridine $(2.15 \mathrm{~mL})$, pyridine $(10.75 \mathrm{~mL})$, and THF $(29 \mathrm{~mL})]$. After $1 \mathrm{~h}$ at ambient temperature the reaction was poured into water $(50 \mathrm{~mL})$ and extracted with $\mathrm{Et}_{2} \mathrm{O}(3 \times 50 \mathrm{~mL})$. The combined organic phase was dried over $\mathrm{MgSO}_{4}$, filtered, concentrated, and purified by FC (1/9 EtOAc/Hex) to afford $264 \mathrm{mg}(88 \%)$ of $\mathbf{7 b} .[\alpha]_{\mathrm{D}}=+1.3\left(\mathrm{CHCl}_{3}, c\right.$ 1.0). IR (film) 3498, 2933, 2858, 1739, 1607, 1580, 1474, 1454, 1290, 1267, 1210, 1092, 911, $737 \mathrm{~cm}^{-1} ;{ }^{1} \mathrm{H}$ NMR $\left(400 \mathrm{MHz}, \mathrm{CDCl}_{3}\right) \delta 7.47-7.52(4 \mathrm{H}, \mathrm{m}), 7.18-7.30(12 \mathrm{H}, \mathrm{m})$, $6.90(1 \mathrm{H}, \mathrm{d}, J=8.0 \mathrm{~Hz}), 6.75(1 \mathrm{H}, \mathrm{d}, J=7.2 \mathrm{~Hz}), 5.07(1 \mathrm{H}, \mathrm{d}, J=12.8 \mathrm{~Hz}), 4.98(1 \mathrm{H}, \mathrm{d}, J=12.4 \mathrm{~Hz}), 4.10-4.17$ $(1 \mathrm{H}, \mathrm{m}), 3.60-3.67(1 \mathrm{H}, \mathrm{m}), 3.43(1 \mathrm{H}, \mathrm{d}, J=10.0 \mathrm{~Hz}), 3.05-3.18(3 \mathrm{H}, \mathrm{m}), 2.93-3.01(1 \mathrm{H}, \mathrm{m}), 2.83-2.92(1 \mathrm{H}, \mathrm{m})$, $1.68-1.79(3 \mathrm{H}, \mathrm{m}), 1.61-1.65(2 \mathrm{H}, \mathrm{m}), 1.30-1.56(12 \mathrm{H}, \mathrm{m}), 0.96-1.24(8 \mathrm{H}, \mathrm{m}), 1.13(3 \mathrm{H}, \mathrm{s}), 1.05(3 \mathrm{H}, \mathrm{s}), 0.81$ $(9 \mathrm{H}, \mathrm{s}), 0.77(3 \mathrm{H}, \mathrm{d}, J=6.4 \mathrm{~Hz}),-0.04(6 \mathrm{H}, \mathrm{s}) ;{ }^{13} \mathrm{C} \mathrm{NMR}\left(75 \mathrm{MHz}, \mathrm{CDCl}_{3}\right) \delta 176.7,160.4,157.7,148.5,140.0$, $136.5,135.4,129.2,128.8,128.6,128.1,128.0,127.8,126.8,125.6,117.3,115.6,113.6,106.0,82.2,78.4,77.8$, $75.2,71.9,68.6,66.2,46.9,42.6,39.1,36.4,36.3,34.3,32.2,31.8,31.7,28.2,26.8,26.0,25.2,23.9,23.8,21.4$, 20.4, 18.1, 15.2, -4.5, -4.8. MS (ES) $m / z(\%): 969.50\left([\mathrm{MNa}]^{+}, 65\right)$.

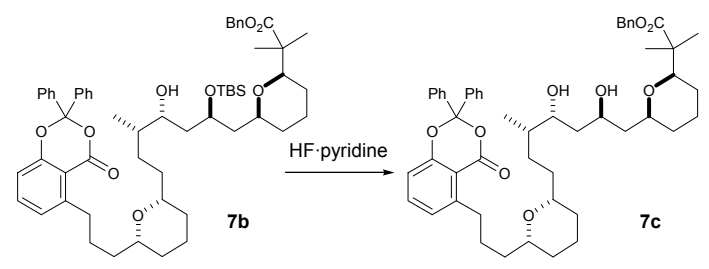

Compound 7c. To a stirred solution of silyl ether $7 \mathbf{b}(20 \mathrm{mg}, 0.021$ mmol) was added pyridine $(0.020 \mathrm{~mL}, 0.25 \mathrm{mmol})$ followed by $70 \%$ HF.pyridine $(0.020 \mathrm{~mL})$. The reaction was allowed to stir for $10 \mathrm{~h}$ at room temperature. The reaction was diluted with $\mathrm{Et}_{2} \mathrm{O}(20 \mathrm{~mL})$ and extracted with $\mathrm{Et}_{2} \mathrm{O}(3 \times 20 \mathrm{~mL})$ from saturated $\mathrm{CuSO}_{4}(20 \mathrm{~mL})$. The combined organic phase was dried over $\mathrm{Na}_{2} \mathrm{SO}_{4}$, filtered, and purified by FC $(1 / 3 \mathrm{EtOAc} / \mathrm{Hex})$ to afford $11.5 \mathrm{mg}(66 \%)$ of diol 7c. $[\alpha]_{\mathrm{D}}=+2.4\left(\mathrm{CHCl}_{3}, c\right.$ 0.58). IR (film) 3500 , 2936, 1732, 1607, 1581, 1455, 1267, 1207, 1093, 1046, $912 \mathrm{~cm}^{-1} ;{ }^{1} \mathrm{H}$ NMR $\left(400 \mathrm{MHz}, \mathrm{CDCl}_{3}\right) \delta 7.55-7.59(4 \mathrm{H}$, m), 7.27-7.38 (12H, m), $6.98(1 \mathrm{H}, \mathrm{d}, J=8.4 \mathrm{~Hz}), 6.82(1 \mathrm{H}, \mathrm{d}, J=7.6 \mathrm{~Hz}), 5.17(1 \mathrm{H}, \mathrm{d}, J=12.4 \mathrm{~Hz}), 5.08(1 \mathrm{H}, \mathrm{d}, J$ $=12.4 \mathrm{~Hz}), 4.04-4.10(1 \mathrm{H}, \mathrm{m}), 3.73(1 \mathrm{H}, \mathrm{app} \mathrm{t}, J=6.8 \mathrm{~Hz}), 3.61(1 \mathrm{H}, \mathrm{d}, J=11.2 \mathrm{~Hz}), 3.53-3.59(1 \mathrm{H}, \mathrm{m}), 3.13-$

\footnotetext{
${ }^{2}$ Soltani, O.; De Brabander, J. K. Angew. Chem. Int. Ed., 2005, 44, 1696-1699.
} 
$3.22(2 \mathrm{H}, \mathrm{m}), 2.97-3.22(1 \mathrm{H}, \mathrm{m}), 2.91-2.97(1 \mathrm{H}, \mathrm{m}), 1.68-1.88(3 \mathrm{H}, \mathrm{m}), 1.54-1.64(4 \mathrm{H}, \mathrm{m}), 1.38-1.54(12 \mathrm{H}, \mathrm{m})$, 0.99-1.32 (6H, m), $1.19(3 \mathrm{H}, \mathrm{s}), 1.15(3 \mathrm{H}, \mathrm{s}), 0.85(3 \mathrm{H}, \mathrm{d}, J=6.8 \mathrm{~Hz}) ;{ }^{13} \mathrm{C}$ NMR $\left(75 \mathrm{MHz}, \mathrm{CDCl}_{3}\right) \delta 176.6,160.5$, 157.7, 148.6, 140.0, 136.4, 135.5 129.3, 129.2, 128.7, 128.6, 126.7, 125.7, 115.6, 113.6, 106.0, 83.1, 80.4, 78.5, $77.8,72.5,70.7,66.7,46.8,42.6,39.1,38.9,36.3,34.4,34.3,32.1,31.8,31.7,28.3,26.9,25.0,24.0,23.4,21.9$, 20.1, 15.4. MS (ES) $\left.m / z(\%): 833.50\left([\mathrm{MH}]^{+}, 100\right), 855.45\left([\mathrm{MNa}]^{+}\right), 40\right)$.

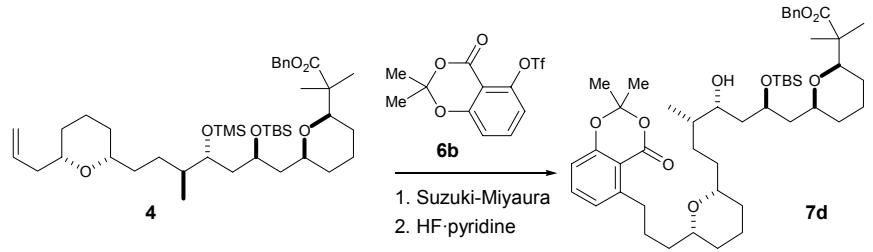

Compound 7d. To a round bottom flask charged with bis-silyl ether 4 (200 $\mathrm{mg}, 0.28 \mathrm{mmol})$ and solid 9-BBN (41 mg, $0.34 \mathrm{mmol}$ ) was added freshly distilled THF $(1.0 \mathrm{~mL})$. The reaction was allowed to stir for $17 \mathrm{~h}$ at ambient temperature. Then a degassed solution of $3 \mathrm{M} \mathrm{K}_{3} \mathrm{PO}_{4}(0.23 \mathrm{~mL}, 0.70 \mathrm{mmol})$ was added, followed by a solution of triflate $6 \mathbf{b}^{3}(136 \mathrm{mg}, 0.42 \mathrm{mmol})$ and $\mathrm{PdCl}_{2} \mathrm{dppf}(12 \mathrm{mg}, 0.014 \mathrm{mmol})$ in degassed DMF $(1.5 \mathrm{~mL})$. The reaction was allowed to stir for $12 \mathrm{~h}$ at ambient temperature at which time the reaction was diluted with $\mathrm{Et}_{2} \mathrm{O}(20 \mathrm{~mL})$ and extracted with $\mathrm{Et}_{2} \mathrm{O}(3 \times 30 \mathrm{~mL})$ from water $(30 \mathrm{~mL})$. The organic phase was dried over $\mathrm{MgSO}_{4}$, filtered, concentrated, and purified by FC (5/95 EtOAc/Hex) to yield $163 \mathrm{mg}(65 \%)$ of coupled bissilyl ether which was taken up in THF $(5 \mathrm{~mL})$ and treated with HF-pyridine $[1 \mathrm{~mL}$ from a stock solution composed of $70 \%$ HF-pyridine $(2.15 \mathrm{~mL})$, pyridine $(10.75 \mathrm{~mL})$, and THF $(29 \mathrm{~mL})]$ for $0.5 \mathrm{~h}$. The reaction was then quenched by addition of saturated $\mathrm{NaHCO}_{3}(10 \mathrm{~mL})$ and extracted with $\mathrm{Et}_{2} \mathrm{O}(3 \times 40 \mathrm{~mL})$ from water $(30 \mathrm{~mL})$. The organic phase was dried over $\mathrm{MgSO}_{4}$, filtered, concentrated, and purified by FC (13/87 EtOAc/Hex) to afford $140 \mathrm{mg}$ $(95 \%)$ of 7 d. $[\alpha]_{\mathrm{D}}=-0.56\left(\mathrm{CHCl}_{3}, c 0.80\right)$. IR (film) 3514, 2933, 2858, 1739, 1606, 1582, 1476, 1389, 1314, 1269, 1210, 1086, 1046, $836 \mathrm{~cm}^{-1}$; ${ }^{1} \mathrm{H}$ NMR $\left(400 \mathrm{MHz}, \mathrm{CDCl}_{3}\right) \delta 7.27-7.39(6 \mathrm{H}, \mathrm{m}), 6.92(1 \mathrm{H}, \mathrm{d}, J=7.6 \mathrm{~Hz}), 6.77(1 \mathrm{H}$, $\mathrm{d}, J=8.0 \mathrm{~Hz}), 5.13(1 \mathrm{H}, \mathrm{d}, J=12.4 \mathrm{~Hz}), 5.04(1 \mathrm{H}, \mathrm{d}, J=12.8 \mathrm{~Hz}), 4.16-4.23(1 \mathrm{H}, \mathrm{m}), 3.66-3.72(1 \mathrm{H}, \mathrm{m}), 3.49$ $(1 \mathrm{H}, \mathrm{s}), 3.48(1 \mathrm{H}, \mathrm{d}, J=9.6 \mathrm{~Hz}), 3.16-3.30(3 \mathrm{H}, \mathrm{m}), 3.04-3.12(2 \mathrm{H}, \mathrm{m}), 1.72-1.86(3 \mathrm{H}, \mathrm{m}), 1.68(6 \mathrm{H}, \mathrm{s}), 1.39-1.64$ $(16 \mathrm{H}, \mathrm{m}), 1.08-1.24(6 \mathrm{H}, \mathrm{m}), 1.18(3 \mathrm{H}, \mathrm{s}), 1.11(3 \mathrm{H}, \mathrm{s}), 0.86(9 \mathrm{H}, \mathrm{s}), 0.82(3 \mathrm{H}, \mathrm{d}, J=6.8 \mathrm{~Hz}), 0.05(6 \mathrm{H}, \mathrm{s}) ;{ }^{13} \mathrm{C}$ NMR $\left(75 \mathrm{MHz}, \mathrm{CDCl}_{3}\right) \delta 176.8,160.4,157.3,148.4,136.5,128.7,128.2,127.8,125.4,115.3,112.3,105.1,82.2$, 78.4, 77.9, 75.3, 72.0, 68.7, 66.3, 47.0, 42.6, 39.1, 36.6, 36.4, 34.4, 34.3, 32.2, 31.9, 31.7, 28.2, 27.3, 26.0, 25.9, $25.8,25.2,24.0,23.8,21.4,20.5,18.1,15.2,-4.5,-4.8$. MS (ES) $m / z(\%): 845.40\left([\mathrm{MNa}]^{+}, 100\right)$,

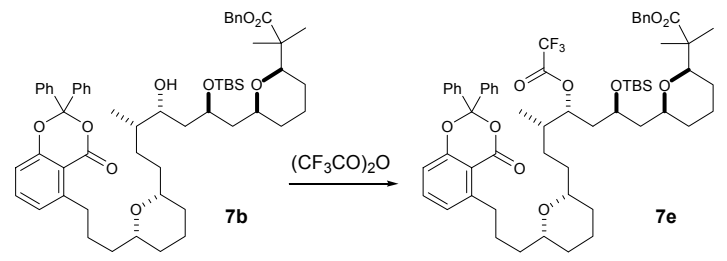

Trifluoroacetate 7e. To a stirred solution alcohol $7 \mathbf{b}(95 \mathrm{mg}, 0.10$ $\mathrm{mmol})$ in $\mathrm{CH}_{2} \mathrm{Cl}_{2}$ :pyridine $(6 \mathrm{~mL}, 5: 1)$ at $0{ }^{\circ} \mathrm{C}$ was added trifluoroacetic anhydride $(55 \mu \mathrm{L}, 0.30 \mathrm{mmol})$. After $0.5 \mathrm{~h}$, the reaction was poured into water $(50 \mathrm{~mL})$ and extracted with $\mathrm{Et}_{2} \mathrm{O}(3$ $\times 25 \mathrm{~mL}$ ). The combined organic phase was dried over $\mathrm{MgSO}_{4}$, filtered, concentrated, and purified by FC (1/9 EtOAc/Hex) to afford $96 \mathrm{mg}(92 \%)$ of 7e. $[\alpha]_{\mathrm{D}}=+14.0\left(\mathrm{CHCl}_{3}, c 0.83\right)$. IR (film) 2934, 2858, 1779, 1741, 1607, 1581, 1475, 1455, 1266, 1217, 1166, 1090, 912, $836 \mathrm{~cm}^{-1}$; ${ }^{1} \mathrm{H}$ NMR $\left(400 \mathrm{MHz}, \mathrm{CDCl}_{3}\right) \delta 7.54-7.58(4 \mathrm{H}, \mathrm{m}), 7.27-7.38(12 \mathrm{H}, \mathrm{m})$, $6.98(1 \mathrm{H}, \mathrm{d}, J=8.4 \mathrm{~Hz}), 6.82(1 \mathrm{H}, \mathrm{d}, J=8.0 \mathrm{~Hz}), 5.18(1 \mathrm{H}$, app dd, $J=4.0,10.0 \mathrm{~Hz}), 5.10(2 \mathrm{H}, \mathrm{s}), 3.88(1 \mathrm{H}$, app t, $J=9.2 \mathrm{~Hz}), 3.43(1 \mathrm{H}, \mathrm{d}, J=11.2 \mathrm{~Hz}), 3.33(1 \mathrm{H}$, app t, $J=14 \mathrm{~Hz}), 3.11-3.24(2 \mathrm{H}, \mathrm{m}), 3.01-3.10(1 \mathrm{H}, \mathrm{m}), 2.88-$ $2.99(1 \mathrm{H}, \mathrm{m}), 1.68-1.96(5 \mathrm{H}, \mathrm{m}), 1.30-1.61(12 \mathrm{H}, \mathrm{m}), 0.97-1.29(8 \mathrm{H}, \mathrm{m}), 1.21(3 \mathrm{H}, \mathrm{s}), 1.14(3 \mathrm{H}, \mathrm{s}), 0.85-0.89$ $(12 \mathrm{H}, \mathrm{m}), 0.02(3 \mathrm{H}, \mathrm{s}),-0.03(3 \mathrm{H}, \mathrm{s}) ;{ }^{13} \mathrm{C} \mathrm{NMR}\left(75 \mathrm{MHz}, \mathrm{CDCl}_{3}\right) \delta 176.5,160.4,157.7,157.0,148.4,140.0$, $136.5,135.4,129.2,128.6,128.1,127.9,126.7,126.6,125.7,115.6,113.6,106.0,82.1,80.5,77.8,77.6,74.8,66.1$, 65.6, 46.8, 45.2, 42.1, 36.2, 35.9, 35.8, 34.3, 34.0, 32.1, 31.8, 31.7, 28.6, 27.3, 26.7, 26.1, 25.8, 23.9, 23.8, 22.7, 19.7, 18.1, -3.8, -5.0. MS (ES) $m / z(\%): 1065.65\left([\mathrm{MNa}]^{+}, 100\right)$.

\footnotetext{
${ }^{3}$ Fürstner, A.; Konetzki, I. Tetrahedron, 1996, 52, 15071-15078.
} 


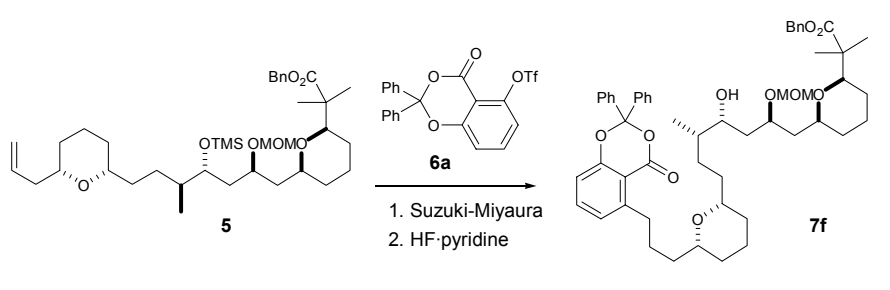

Compound 7f. To a round bottom flask containing olefin $5(281 \mathrm{mg}, 0.43 \mathrm{mmol})$ and solid 9-BBN dimer (160 $\mathrm{mg}, 1.3 \mathrm{mmol})$ was added freshly distilled THF (2 $\mathrm{mL}$ ). The reaction was allowed to stir for $12 \mathrm{~h}$ at ambient temperature. Then a degassed aqueous solution of $3 \mathrm{M} \mathrm{K}_{3} \mathrm{PO}_{4}(0.43 \mathrm{~mL}, 1.29 \mathrm{mmol})$ was added dropwise, followed by a solution of $\mathbf{6 a}(290 \mathrm{mg}, 0.65$

mmol), and $\mathrm{PdCl}_{2} \mathrm{dppf}(17.5 \mathrm{mg}, 0.022 \mathrm{mmol})$ in degassed DMF $(2 \mathrm{~mL})$. The reaction was allowed to stir for $20 \mathrm{~h}$ at ambient temperature, followed by the addition of water $(40 \mathrm{~mL})$ and extraction with $\mathrm{Et}_{2} \mathrm{O}(3 \times 30 \mathrm{~mL})$. The combined organic phase was dried over $\mathrm{MgSO}_{4}$, filtered, concentrated, and filtered through a short pad of silica (8/92 EtOAc/Hex). The crude filtrate was concentrated, taken up in THF $(20 \mathrm{~mL})$, and treated with HF-pyridine [3 $\mathrm{mL}$ from a stock solution composed of $70 \%$ HF.pyridine $(2.15 \mathrm{~mL})$, pyridine $(10.75 \mathrm{~mL})$, and THF $(29 \mathrm{~mL}]$. The reaction was allowed to stir at ambient temperature for $2 \mathrm{~h}$ at which time the mixture was diluted with water (50 $\mathrm{mL})$, and extracted with $\mathrm{Et}_{2} \mathrm{O}(3 \times 50 \mathrm{~mL})$. The combined organic phase was dried over $\mathrm{MgSO}_{4}$, filtered, concentrated, and purified by FC (1/4 EtOAc/Hex) to afford $301 \mathrm{mg}(80 \%$ for 2 steps $)$ of alcohol $7 \mathbf{f}$. $[\alpha]_{\mathrm{D}}=-1.2$ $\left(\mathrm{CHCl}_{3}, c\right.$ 0.18). IR (film) 3520, 2934, 2860, 1739, 1607, 1580, 1476, 1454, 1267, 1093, 1039, $917 \mathrm{~cm}^{-1}$; ${ }^{1} \mathrm{H}$ NMR $\left(400 \mathrm{MHz}, \mathrm{CDCl}_{3}\right) \delta 7.54-7.58(4 \mathrm{H}, \mathrm{m}), 7.26-7.37(12 \mathrm{H}, \mathrm{m}), 6.70(1 \mathrm{H}, \mathrm{d}, J=8.0 \mathrm{~Hz}), 6.81(1 \mathrm{H}, \mathrm{d}, J=7.6 \mathrm{~Hz})$, $5.11(2 \mathrm{H}, \mathrm{s}), 4.60(1 \mathrm{H}, \mathrm{d}, J=6.8 \mathrm{~Hz}), 4.57(1 \mathrm{H}, \mathrm{d}, J=6.4 \mathrm{~Hz}), 3.91-3.98(1 \mathrm{H}, \mathrm{m}), 3.61-3.68(1 \mathrm{H}, \mathrm{m}), 3.50(1 \mathrm{H}, \mathrm{d}$, $J=11.2 \mathrm{~Hz}), 3.28-3.38(1 \mathrm{H}, \mathrm{m}), 3.36(1 \mathrm{H}, \mathrm{s}), 3.34(3 \mathrm{H}, \mathrm{s}), 3.12-3.22(2 \mathrm{H}, \mathrm{m}), 3.00-3.07(1 \mathrm{H}, \mathrm{m}), 2.91-2.97(1 \mathrm{H}$, m), 1.74-1.87 (3H, m), 1.37-1.64 (16H, m), 0.98-1.28 (7H, m), $1.19(3 \mathrm{H}, \mathrm{s}), 1.12(3 \mathrm{H}, \mathrm{s}), 0.86(3 \mathrm{H}, \mathrm{d}, J=6.8 \mathrm{~Hz})$; ${ }^{13} \mathrm{C}$ NMR $\left(100 \mathrm{MHz}, \mathrm{CDCl}_{3}\right) \delta 176.9,160.4,157.7,148.5,140.0,136.6,135.4,129.2,129.1,128.6,128.1,128.0$, $126.8,126.7,125.7,115.6,113.6,106.0,97.5,96.2,82.4,78.4,77.8,75.2,73.6,71.7,66.3,55.9,46.9,41.4,39.1$, $37.2,36.3,34.3,32.1,31.8,31.7,29.9,28.4,26.8,25.3,23.9,23.8,21.4,20.7,15.3$. MS (ES) $\mathrm{m} / z(\%): 899.65$ $\left([\mathrm{MNa}]^{+}, 50\right)$.

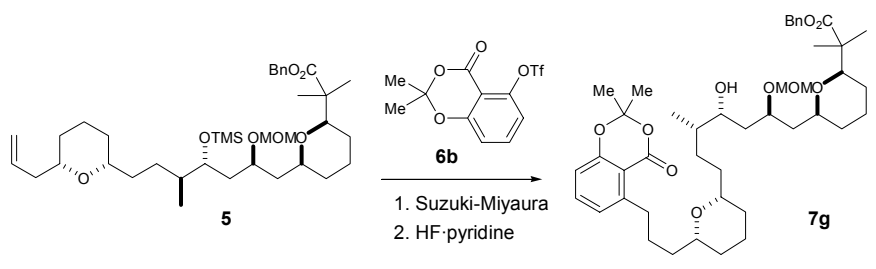

Compound 7g. To an ovendried roundbottom flask charged with silyl ether $5(150 \mathrm{mg}, 0.232 \mathrm{mmol})$ and solid 9-BBN (71 mg, $0.58 \mathrm{mmol})$ was added THF (1 $\mathrm{mL})$. The reaction was allowed to stir under nitrogen at ambient temperature for $14 \mathrm{~h}$. At that time an aqueous solution of $3 \mathrm{M} \mathrm{K}_{3} \mathrm{PO}_{4}(155 \mu \mathrm{L}, 0.464 \mathrm{mmol})$ was added, followed by a solution of $\mathrm{PdCl}_{2} \mathrm{dppf}(9.5 \mathrm{mg}, 0.0116 \mathrm{mmol})$, and $\mathbf{6 b}(113 \mathrm{mg}, 0.348 \mathrm{mg})$ in DMF (1 mL). After 24 $\mathrm{h}$ at ambient temperature the reaction was diluted with water $(30 \mathrm{~mL})$, and extracted with $\mathrm{Et}_{2} \mathrm{O}(2 \times 50 \mathrm{~mL})$. The combined organic phase was dried over $\mathrm{MgSO}_{4}$, filtered, concentrated, and filtered through a short pad of silicagel (1/4 EtOAc/Hex). After concentration, the residue was treated with HF-pyridine [5 $\mathrm{mL}$ from a stock solution composed of $70 \%$ HF.pyridine $(2.15 \mathrm{~mL})$, pyridine $(10.75 \mathrm{~mL})$, and THF $(29 \mathrm{~mL})]$. After $1.5 \mathrm{~h}$ the reaction was poured into water $(30 \mathrm{~mL})$, and extracted with $\mathrm{Et}_{2} \mathrm{O}(2 \times 40 \mathrm{~mL})$. The combined organic phase was dried over $\mathrm{MgSO}_{4}$, filtered, concentrated, and purified by $\mathrm{FC} \mathrm{(3/7,} \mathrm{EtOAc/Hex)} \mathrm{to} \mathrm{afford} 148 \mathrm{mg}$ (85\% for two steps) of $7 \mathbf{g}$. $[\alpha]_{\mathrm{D}}=+2.0\left(\mathrm{CHCl}_{3}, c\right.$ 1.7). IR (film) 3402, 2518, 1655, 1456, 1173, 1031, $730 \mathrm{~cm}^{-1} ;{ }^{1} \mathrm{H}$ NMR $\left(400 \mathrm{MHz}, \mathrm{CDCl}_{3}\right) \delta$ $7.28(1 \mathrm{H}, \mathrm{t}, J=8.0 \mathrm{~Hz}), 7.27-7.40(5 \mathrm{H}, \mathrm{m}), 6.93(1 \mathrm{H}, \mathrm{d}, J=7.2 \mathrm{~Hz}), 6.78(1 \mathrm{H}, \mathrm{d}, J=8.0 \mathrm{~Hz}), 5.12(2 \mathrm{H}, \mathrm{s}), 4.61$ $(1 \mathrm{H}, \mathrm{d}, J=6.4 \mathrm{~Hz}), 4.58(1 \mathrm{H}, \mathrm{d}, J=6.4 \mathrm{~Hz}), 3.90-4.00(1 \mathrm{H}, \mathrm{m}), 3.63-3.69(1 \mathrm{H}, \mathrm{m}), 3.51(1 \mathrm{H}, \mathrm{d}, J=10.0 \mathrm{~Hz})$, 3.17-3.40 (2H, m), $3.35(3 \mathrm{H}, \mathrm{s}), 3.06-3.13(2 \mathrm{H}, \mathrm{m}), 2.95(1 \mathrm{H}, \mathrm{m}), 1.74-1.88(3 \mathrm{H}, \mathrm{m}), 1.69(6 \mathrm{H}, \mathrm{s}), 1.39-1.65$ $(16 \mathrm{H}, \mathrm{m}), 1.07-1.28(7 \mathrm{H}, \mathrm{m}), 1.20(3 \mathrm{H}, \mathrm{s}), 1.12(3 \mathrm{H}, \mathrm{s}), 0.86(3 \mathrm{H}, \mathrm{d}, J=6.8 \mathrm{~Hz}) ;{ }^{13} \mathrm{C}$ NMR $\left(75 \mathrm{MHz}, \mathrm{CDCl}_{3}\right) \delta$ $176.9,160.4,157.3,148.4,136.6,135.3,128.6,128.1,127.9,125.4,115.3,112.2,105.1,96.2,82.4,78.5,77.8$, 75.2, 73.6, 71.7, 66.3, 56.0, 46.9, 41.4, 39.1, 37.2, 36.6, 34.4, 34.3, 32.0, 31.9, 31.7, 28.4, 27.3, 25.9, 25.8, 25.3, 23.9, 23.8, 21.4, 20.7, 15.3. MS (ES) $m / z(\%): 775.40\left([\mathrm{MNa}]^{+}, 100\right)$. 

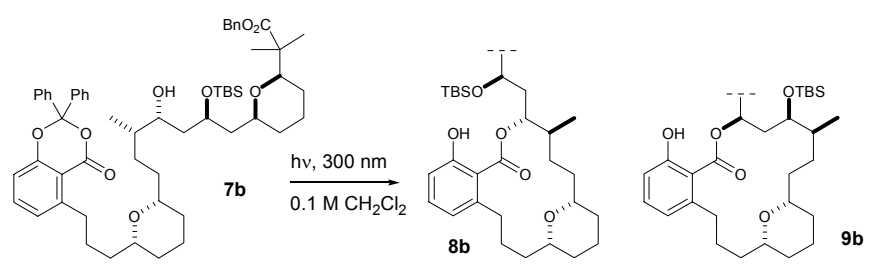

Photolysis of $7 \mathbf{b}$ (representative procedure). The starting material $\mathbf{7 b}$ was first dried via azeotropic removal of water with benzene $(2 \times 1 \mathrm{~mL})$ under vacuo. Then $\mathbf{7 b}$ was taken up in freshly distilled $\mathrm{CH}_{2} \mathrm{Cl}_{2}(0.3 \mathrm{~mL})$ and transferred under nitrogen to a flame dried borosilicate test tube fitted with a rubber septum. This solution was then photolyzed for $1 \mathrm{~h}$ at $300 \mathrm{~nm}$. The solvent was then removed and the crude mixture was purified directly by FC $(3 / 97 \rightarrow 15 / 85$ EtOAc/Hex gradient) to afford $11 \mathrm{mg}$ $(45 \%)$ of $8 \mathbf{b}$, and $2.2 \mathrm{mg}(9 \%)$ of $9 \mathbf{b}$.

8b: $[\alpha]_{\mathrm{D}}=-7.6\left(\mathrm{CHCl}_{3}, c\right.$ 0.55). IR (film) 3401, 2931, 2856, 1725, 1654, 1456, 1259, 1089, 1048, $836 \mathrm{~cm}^{-1} ;{ }^{1} \mathrm{H}$ $\operatorname{NMR}\left(400 \mathrm{MHz}, \mathrm{CDCl}_{3}\right) \delta 9.01(1 \mathrm{H}, \mathrm{s}), 7.25-7.37(4 \mathrm{H}, \mathrm{m}), 7.15-7.22(2 \mathrm{H}, \mathrm{m}), 6.77(1 \mathrm{H}, \mathrm{d}, J=8.4 \mathrm{~Hz}), 6.75(1 \mathrm{H}$, $\mathrm{d}, J=7.6 \mathrm{~Hz}), 5.12-5.18(1 \mathrm{H}, \mathrm{m}), 5.10(1 \mathrm{H}, \mathrm{d}, J=12.8 \mathrm{~Hz}), 5.05(1 \mathrm{H}, \mathrm{d}, J=12.8 \mathrm{~Hz}), 3.98-4.04(1 \mathrm{H}, \mathrm{m}), 3.45$ $(1 \mathrm{H}, \mathrm{dd}, J=1.6,11.2 \mathrm{~Hz}), 3.19-3.38(4 \mathrm{H}, \mathrm{m}), 2.73(1 \mathrm{H}, \mathrm{td}, J=3.6,13.6 \mathrm{~Hz}), 2.14-2.23(1 \mathrm{H}, \mathrm{m}), 1.62-1.89(6 \mathrm{H}$, m), 1.32-1.58 (12H, m), 1.03-1.31 (6H, m), $1.10(3 \mathrm{H}, \mathrm{s}), 1.08(3 \mathrm{H}, \mathrm{s}), 0.88(9 \mathrm{H}, \mathrm{s}), 0.86-0.88(3 \mathrm{H}, \mathrm{m}), 0.07(3 \mathrm{H}$, s), $0.04(3 \mathrm{H}, \mathrm{s}) ;{ }^{13} \mathrm{C}$ NMR $\left(75 \mathrm{MHz}, \mathrm{CDCl}_{3}\right) \delta 177.4,169.5,157.9,145.0,132.4,128.8,128.7,128.1,127.8,127.5$, $121.1,117.8,115.2,82.1,80.2,78.7,77.3,75.1,66.8,66.4,47.0,44.9,38.0,37.0,36.1,33.7,33.1,32.7,32.6,32.2$, 31.5, 28.7, 26.3, 26.2, 25.4, 24.3, 23.8, 21.1, 21.0, 18.4, 14.9, -4.0, -4.6. MS (ES) $m / z(\%): 787.50$ ([MNa $\left.]^{+}, 100\right)$. 9b: ${ }^{1} \mathrm{H}$ NMR $\left(400 \mathrm{MHz}, \mathrm{CDCl}_{3}\right) \delta 11.24(1 \mathrm{H}, \mathrm{s}), 7.24-7.36(6 \mathrm{H}, \mathrm{m}), 6.82(1 \mathrm{H}, \mathrm{d}, J=7.2 \mathrm{~Hz}), 6.69(1 \mathrm{H}, \mathrm{d}, J=7.2$ $\mathrm{Hz}), 5.52-5.58(1 \mathrm{H}, \mathrm{m}), 5.10(2 \mathrm{H}, \mathrm{s}), 3.73-3.76(1 \mathrm{H}, \mathrm{m}), 3.48(1 \mathrm{H}, \mathrm{d}, J=10.0 \mathrm{~Hz}), 3.40-3.47(1 \mathrm{H}, \mathrm{m}), 3.26(1 \mathrm{H}$, app t, $J=9.2 \mathrm{~Hz}), 3.13-3.21(1 \mathrm{H}, \mathrm{m}), 3.00-3.06(1 \mathrm{H}, \mathrm{m}), 2.73-2.80(1 \mathrm{H}, \mathrm{m}), 1.92-1.99(3 \mathrm{H}, \mathrm{m}), 1.72-1.86(5 \mathrm{H}$, m), 1.34-1.62 (12H, m), 1.06-1.30 (5H, m), $1.23(3 \mathrm{H}, \mathrm{s}), 1.14(3 \mathrm{H}, \mathrm{s}), 0.88(9 \mathrm{H}, \mathrm{s}), 0.85(3 \mathrm{H}, \mathrm{d}, J=6.4 \mathrm{~Hz}), 0.03$ $(6 \mathrm{H}, \mathrm{s})$. MS (ES) $m / z(\%): 787.45\left([\mathrm{MNa}]^{+}, 100\right)$.
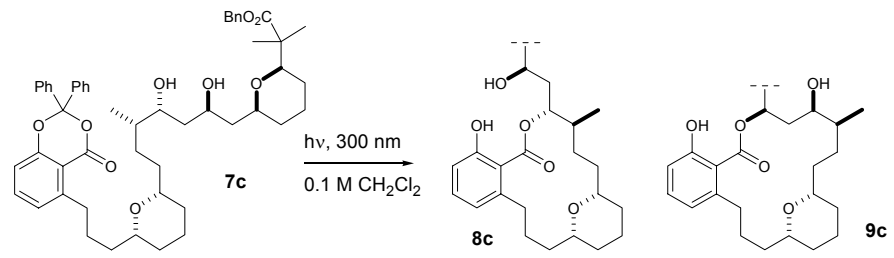

Photolysis of 7c. According to the general procedure, compound $7 \mathbf{c}$ was photolyzed to produce a mixture of lactones $8 \mathbf{c}(26 \%)$ and $9 \mathbf{c}(12 \%)$, and recovered starting material $7 \mathbf{c}(30 \%)$.

8c: $[\alpha]_{\mathrm{D}}=-6.0\left(\mathrm{CHCl}_{3}, c\right.$ 0.11). IR (film) 3369, 2931, $2857,1723,1606,1580,1455,1262,1092,1047 \mathrm{~cm}^{-1}$;

${ }^{1} \mathrm{H}$ NMR $\left(400 \mathrm{MHz}, \mathrm{CDCl}_{3}\right) \delta 9.33(1 \mathrm{H}, \mathrm{br} \mathrm{s}), 7.28-7.36(5 \mathrm{H}, \mathrm{m}), 7.21(1 \mathrm{H}, \mathrm{t}, J=8.0 \mathrm{~Hz}), 6.74(2 \mathrm{H}, \mathrm{d}, J=8.0 \mathrm{~Hz})$, $5.22(1 \mathrm{H}, \mathrm{ddd}, J=3.2,8.8,8.8 \mathrm{~Hz}), 5.15(1 \mathrm{H}, \mathrm{d}, J=12.4 \mathrm{~Hz}), 5.11(1 \mathrm{H}, \mathrm{d}, J=12.8 \mathrm{~Hz}), 3.87-3.95(1 \mathrm{H}, \mathrm{m}), 3.56$ $(1 \mathrm{H}, \mathrm{d}, J=10.8 \mathrm{~Hz}), 3.48-3.55(1 \mathrm{H}, \mathrm{m}), 3.31(1 \mathrm{H}$, app t, $J=10.0 \mathrm{~Hz}), 3.25(1 \mathrm{H}$, app t, $J=10.4 \mathrm{~Hz}), 3.11-3.20$ $(1 \mathrm{H}, \mathrm{m}), 2.79(1 \mathrm{H}, \mathrm{td}, J=3.6,12.4 \mathrm{~Hz}), 2.08-2.17(1 \mathrm{H}, \mathrm{m}), 1.08-1.88(24 \mathrm{H}, \mathrm{m}), 1.15(3 \mathrm{H}, \mathrm{s}), 1.11(3 \mathrm{H}, \mathrm{s}), 0.90$ $(3 \mathrm{H}, \mathrm{d}, J=6.8 \mathrm{~Hz})$. MS (ES) $m / z(\%): 651.45\left([\mathrm{MH}]^{+}, 100\right)$.

9c: $[\alpha]_{\mathrm{D}}=-9.2\left(\mathrm{CHCl}_{3}, c\right.$ 0.06). IR (film) 3437, 2931, 2856, 1732, 1652, 1456, 1374, 1252, 1212, 1087, $1049 \mathrm{~cm}^{-1}$; ${ }^{1} \mathrm{H}$ NMR $\left(400 \mathrm{MHz}, \mathrm{CDCl}_{3}\right) \delta 10.24(1 \mathrm{H}, \mathrm{s}), 7.23-7.35(6 \mathrm{H}, \mathrm{m}), 6.82(1 \mathrm{H}, \mathrm{d}, J=8.4 \mathrm{~Hz}), 6.69(1 \mathrm{H}, \mathrm{d}, J=6.8 \mathrm{~Hz})$, 5.61-5.69 $(1 \mathrm{H}, \mathrm{m}), 5.18(1 \mathrm{H}, \mathrm{d}, J=12.4 \mathrm{~Hz}), 5.10(1 \mathrm{H}, \mathrm{d}, J=12.8 \mathrm{~Hz}), 3.52(1 \mathrm{H}, \mathrm{dd}, J=1.8,10.8 \mathrm{~Hz}), 3.48-3.54$ $(1 \mathrm{H}, \mathrm{m}), 3.39-3.46(1 \mathrm{H}, \mathrm{m}), 3.23(1 \mathrm{H}$, app t, $J=9.6 \mathrm{~Hz}), 3.12(1 \mathrm{H}$, app t, $J=10.0 \mathrm{~Hz}), 2.98-3.06(1 \mathrm{H}, \mathrm{m}), 2.84-$ $2.92(1 \mathrm{H}, \mathrm{m}), 1.98-2.06(2 \mathrm{H}, \mathrm{m}), 1.70-1.89(4 \mathrm{H}, \mathrm{m}), 1.08-1.70(19 \mathrm{H}, \mathrm{m}), 1.19(3 \mathrm{H}, \mathrm{s}), 1.11(3 \mathrm{H}, \mathrm{s}), 0.83(3 \mathrm{H}, \mathrm{d}, J$ $=6.8 \mathrm{~Hz}) . \mathrm{MS}(\mathrm{ES}) \mathrm{m} / \mathrm{z}(\%): 651.40\left([\mathrm{MH}]^{+}, 100\right)$.

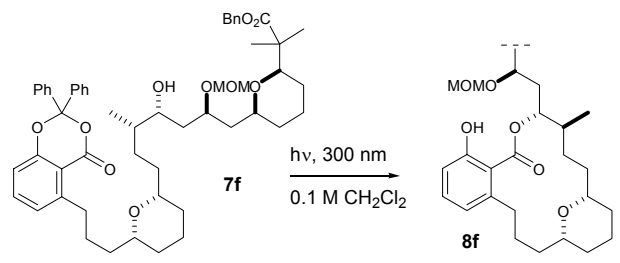

Photolysis of $7 \mathbf{f}$. According to the general procedure, compound $\mathbf{7 f}$ was photolyzed to produce lactone $\mathbf{8 f}(34 \%)$ and recovered starting material $7 \mathbf{f}(16 \%)$.

8f: $[\alpha]_{\mathrm{D}}=-15.3\left(\mathrm{CHCl}_{3}, c\right.$ 0.35). IR (film) 3392, 2933, 1722, 1608, 1456, $1263,1092,1035,919 \mathrm{~cm}^{-1} ;{ }^{1} \mathrm{H}$ NMR $\left(400 \mathrm{MHz}, \mathrm{CDCl}_{3}\right) \delta 8.67(1 \mathrm{H}, \mathrm{s})$, $7.29-7.36(5 \mathrm{H}, \mathrm{m}), 7.19(1 \mathrm{H}, \mathrm{t}, J=8.0 \mathrm{~Hz}), 6.75(2 \mathrm{H}$, app t, $J=8.8 \mathrm{~Hz})$, $5.20(1 \mathrm{H}$, app dd, $J=6.4,9.6 \mathrm{~Hz}), 5.08(2 \mathrm{H}, \mathrm{s}), 4.60(1 \mathrm{H}, \mathrm{d}, J=7.2 \mathrm{~Hz}), 4.56(1 \mathrm{H}, \mathrm{d}, J=6.8 \mathrm{~Hz}), 3.86-3.93(1 \mathrm{H}$, $\mathrm{m}), 3.43(1 \mathrm{H}, \mathrm{d}, J=10.0 \mathrm{~Hz}), 3.20-3.38(4 \mathrm{H}, \mathrm{m}), 3.33(3 \mathrm{H}, \mathrm{s}), 2.60(1 \mathrm{H}, \mathrm{td}, J=4.0,13.6 \mathrm{~Hz}), 2.20-2.28(1 \mathrm{H}, \mathrm{m})$, $1.69-1.93(6 \mathrm{H}, \mathrm{m}), 1.35-1.65(12 \mathrm{H}, \mathrm{m}), 1.03-1.27(6 \mathrm{H}, \mathrm{m}), 1.12(3 \mathrm{H}, \mathrm{s}), 1.08(3 \mathrm{H}, \mathrm{s}), 0.87(3 \mathrm{H}, \mathrm{d}, J=6.8 \mathrm{~Hz}) ;{ }^{13} \mathrm{C}$ 
$\operatorname{NMR}\left(75 \mathrm{MHz}, \mathrm{CDCl}_{3}\right) \delta 177.1,168.9,156.9,144.4,136.5,132.1,128.7,128.1,127.9,121.2,118.4,115.2,96.1$, 82.5, 80.3, 77.4, 77.1, 75.1, 72.7, 66.3, 56.1, 46.9, 40.6, 36.7, 36.0, 33.9, 33.6, 33.0, 32.6, 32.5, 32.1, 31.8, 31.2, 28.5, 25.4, 24.2, 23.8, 21.1, 14.3. MS (ES) $\mathrm{m} / z(\%): 717.45$ ([MNa $\left.]^{+}, 100\right)$.

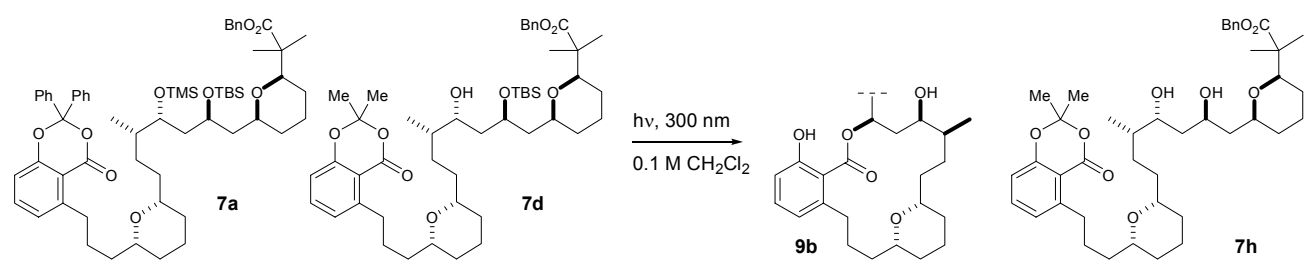

Photolysis of $7 \mathrm{a}$ in the presence of alcohol $7 \mathrm{~d}$. According to the general procedure, photolysis of a mixture of $7 \mathbf{a}$ (1 equiv) and 7d (3 equiv) produced a

mixture of lactone $9 \mathbf{b}(10 \%)$, diol $7 \mathbf{h}(65 \%)$ and recovered starting material $\mathbf{7 d}(10 \%)$.

7h: $[\alpha]_{\mathrm{D}}=-4.4\left(\mathrm{CHCl}_{3}, c\right.$ 0.39). IR (film) 3480, 2935, 2860, 1737, 1606, 1582, 1455, 1314, 1269, 1210, 1083, $1044 \mathrm{~cm}^{-1} ;{ }^{1} \mathrm{H}$ NMR $\left(400 \mathrm{MHz}, \mathrm{CDCl}_{3}\right) \delta 7.27-7.40(6 \mathrm{H}, \mathrm{m}), 6.93(1 \mathrm{H}, \mathrm{d}, J=8.0 \mathrm{~Hz}), 6.78(1 \mathrm{H}, \mathrm{d}, J=8.4 \mathrm{~Hz})$, $5.16(1 \mathrm{H}, \mathrm{d}, J=12.4 \mathrm{~Hz}), 5.08(1 \mathrm{H}, \mathrm{d}, J=12.8 \mathrm{~Hz}), 4.03-4.10(1 \mathrm{H}, \mathrm{m}), 3.83(1 \mathrm{H}, \mathrm{s}), 3.70-3.75(1 \mathrm{H}, \mathrm{m}), 3.61(1 \mathrm{H}$, $\mathrm{d}, J=11.2 \mathrm{~Hz}), 3.53-3.62(1 \mathrm{H}, \mathrm{m}), 3.17-3.30(3 \mathrm{H}, \mathrm{m}), 3.05-3.12(2 \mathrm{H}, \mathrm{m}), 1.72-1.88(4 \mathrm{H}, \mathrm{m}), 1.69(6 \mathrm{H}, \mathrm{s}), 1.53-$ $1.66(8 \mathrm{H}, \mathrm{m}), 1.40-1.52(8 \mathrm{H}, \mathrm{m}), 1.08-1.33(5 \mathrm{H}, \mathrm{m}), 1.19(3 \mathrm{H}, \mathrm{s}), 1.15(3 \mathrm{H}, \mathrm{s}), 0.85(3 \mathrm{H}, \mathrm{d}, J=7.2 \mathrm{~Hz}) ;{ }^{13} \mathrm{C}$ NMR $\left(75 \mathrm{MHz}, \mathrm{CDCl}_{3}\right) \delta 176.7,160.5,157.3,148.5,136.4,135.3,128.7,128.3,125.5,115.3,112.2,105.2,83.1,80.4$, $78.5,77.8,72.5,70.7,66.7,46.8,42.6,39.1,38.9,36.6,34.4,34.3,32.2,31.9,31.8,28.3,27.3,25.9,25.8,25.0$, 24.0, 23.4, 21.9, 20.1, 15.4. MS (ES) $m / z(\%): 731.35\left([\mathrm{MNa}]^{+}, 100\right)$.

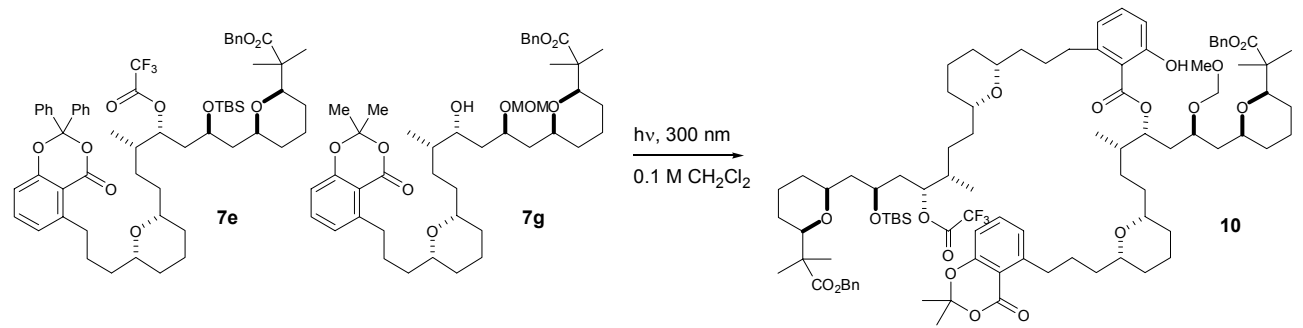

Trifluoroacetate 10. To an ovendried flask was added alcohol 7g (144 mg, 0.192 mmol) and 7 e (100 mg, $0.095 \mathrm{mmol})$. These starting materials were dried via azeotropic removal of water with benzene under vacuo $(3$ $\times 2 \mathrm{~mL})$. The combined starting materials were then dissolved in freshly distilled $\mathrm{CH}_{2} \mathrm{Cl}_{2}(0.48 \mathrm{~mL})$ and transferred to a flame dried borosilicate test tube fitted with a rubber septum under nitrogen. This test tube was sealed with parafilm and the solution was photolyzed for $1 \mathrm{~h}$ at $300 \mathrm{~nm}$ in a Rayonett ${ }^{\mathbb{B}}$ photochemical reactor at ambient temperature. The solvent was then removed and the crude material was purified directly by FC $(3 / 97 \rightarrow 30 / 70$ EtOAc/Hex gradient) to afford $41.4 \mathrm{mg}(27 \%)$ of 10, along with $50 \mathrm{mg}(50 \%)$ of 7 e and $114 \mathrm{mg}(80 \%)$ of $7 \mathrm{~g}$. [ $\alpha]_{\mathrm{D}}$ $=+6.8\left(\mathrm{CHCl}_{3}, c\right.$ 0.83). IR (film) 2935, 2859, 1780, 1737, 1652, 1606, 1581, 1450, 1313, 1269, 1216, 1165, 1087, 1046, $917 \mathrm{~cm}^{-1}$; ${ }^{1} \mathrm{H}$ NMR $\left(400 \mathrm{MHz} \mathrm{CDCl}_{3}\right) \delta 10.98(1 \mathrm{H}, \mathrm{s}), 7.27-7.40(11 \mathrm{H}, \mathrm{m}), 7.23(1 \mathrm{H}, \mathrm{t}, J=8.0 \mathrm{~Hz}), 6.92$ $(1 \mathrm{H}, \mathrm{d}, J=7.6 \mathrm{~Hz}), 6.79(2 \mathrm{H}, \mathrm{dd}, J=3.2,8.0 \mathrm{~Hz}), 6.68(1 \mathrm{H}, \mathrm{d}, J=8.0 \mathrm{~Hz}), 5.42-5.44(1 \mathrm{H}, \mathrm{m}), 5.18(1 \mathrm{H}$, app dd, $J$ $=3.6,10.0 \mathrm{~Hz}), 5.10(2 \mathrm{H}, \mathrm{s}), 5.07(2 \mathrm{H}, \mathrm{s}), 4.58(1 \mathrm{H}, \mathrm{d}, J=6.8 \mathrm{~Hz}), 4.51(1 \mathrm{H}, \mathrm{d}, J=6.8 \mathrm{~Hz}), 3.89(1 \mathrm{H}$, app t, $J=$ $9.2 \mathrm{~Hz}), 3.64-3.74(1 \mathrm{H}, \mathrm{m}), 3.38-3.48(3 \mathrm{H}, \mathrm{m}), 3.28-3.34(1 \mathrm{H}, \mathrm{m}), 3.30(3 \mathrm{H}, \mathrm{s}), 3.14-3.27(4 \mathrm{H}, \mathrm{m}), 3.08(2 \mathrm{H}, \mathrm{t}, J$ $=7.2 \mathrm{~Hz}), 2.84-2.94(2 \mathrm{H}, \mathrm{m}), 1.34-1.96(40 \mathrm{H}, \mathrm{m}), 1.69(6 \mathrm{H}, \mathrm{s}), 1.07-1.28(10 \mathrm{H}, \mathrm{m}), 1.21(3 \mathrm{H}, \mathrm{s}), 1.14(3 \mathrm{H}, \mathrm{s})$, $1.11(3 \mathrm{H}, \mathrm{s}), 1.08(3 \mathrm{H}, \mathrm{s}), 0.95(3 \mathrm{H}, \mathrm{d}, J=6.4 \mathrm{~Hz}), 0.86-0.88(3 \mathrm{H}, \mathrm{m}), 0.85(9 \mathrm{H}, \mathrm{s}), 0.02(3 \mathrm{H}, \mathrm{s}),-0.03(3 \mathrm{H}, \mathrm{s}) ;{ }^{13} \mathrm{C}$ $\operatorname{NMR}\left(75 \mathrm{MHz}, \mathrm{CDCl}_{3}\right) \delta 176.6,176.5,171.0,162.1,160.4,157.3,148.4,145.4,136.6,135.3,133.8,128.7,128.2$, 128.0, 125.4, 122.2, 115.8, 115.3, 113.3, 112.3, 105.1, 96.4, 82.1, 80.5, 77.9, 77.7, 74.9, 74.7, 72.5, 66.2, 65.6, 56.1, 46.8, 45.3, 42.0, 36.9, 36.8, 36.6, 36.1, 36.0, 35.8, 35.7, 34.4, 34.0, 32.2, 31.9, 31.8, 28.9, 28.6, 28.1, 27.3, 26.1, 25.9, 25.8, 23.9, 23.8, 22.8, 22.2, 20.3, 19.7, 18.1, 15.0, 14.3, -3.8, -5.0. MS (ES) $m / z(\%): 1636.55$ ([MNa] $]^{+}$ $35)$. 

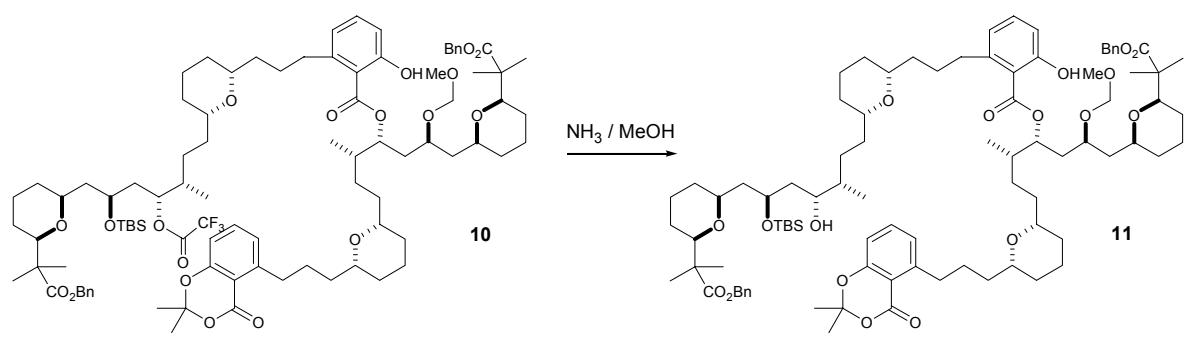

Alcohol 11. To a stirred solution of trifluoroacetate $\mathbf{1 0}(41.4 \mathrm{mg}, 0.025$ $\mathrm{mmol})$ in THF $(5 \mathrm{~mL})$ was added $2.0 \mathrm{M} \mathrm{NH}_{3}$ in $\mathrm{MeOH}(2 \mathrm{~mL})$. The reaction was allowed to stir at ambient temperature for $12 \mathrm{~h}$. The solvent was removed and the crude material was purified by FC $(25 / 75$

EtOAc/Hex) to afford $35.5 \mathrm{mg}(94 \%)$ of alcohol 11. $[\alpha]_{\mathrm{D}}=-1.3\left(\mathrm{CHCl}_{3}, c\right.$ 0.78). IR (film) 3508, 2934, 2858, 1738, 1652, 1606, 1582, 1454, 1313, 1269, 1212, 1087, 1044, $919 \mathrm{~cm}^{-1} ;{ }^{1} \mathrm{H}$ NMR $\left(400 \mathrm{MHz}, \mathrm{CDCl}_{3}\right) \delta 10.99(1 \mathrm{H}, \mathrm{s})$, $7.27-7.40(11 \mathrm{H}, \mathrm{m}), 7.23(1 \mathrm{H}, \mathrm{t}, J=8.0 \mathrm{~Hz}), 6.91(1 \mathrm{H}, \mathrm{d}, J=8.0 \mathrm{~Hz}), 6.78(2 \mathrm{H}, \mathrm{dd}, J=1.6,8.0 \mathrm{~Hz}), 6.68(1 \mathrm{H}, \mathrm{d}, J$ $=7.2 \mathrm{~Hz}), 5.40-5.44(1 \mathrm{H}, \mathrm{m}), 5.03-5.15(4 \mathrm{H}, \mathrm{m}), 4.57(1 \mathrm{H}, \mathrm{d}, J=7.6 \mathrm{~Hz}), 4.51(1 \mathrm{H}, \mathrm{d}, J=6.4 \mathrm{~Hz}), 4.17-4.24(1 \mathrm{H}$, $\mathrm{m}), 3.65-3.72(2 \mathrm{H}, \mathrm{m}), 3.49(1 \mathrm{H}, \mathrm{d}, J=11.2 \mathrm{~Hz}), 3.44(1 \mathrm{H}, \mathrm{d}, J=10.8 \mathrm{~Hz}), 3.36-3.3 .41(1 \mathrm{H}, \mathrm{m}), 3.30(3 \mathrm{H}, \mathrm{s})$, 3.16-3.27 (5H, m), $3.07(2 \mathrm{H}, \mathrm{t}, J=7.2 \mathrm{~Hz}), 2.85-2.91(2 \mathrm{H}, \mathrm{m}), 1.34-1.86(40 \mathrm{H}, \mathrm{m}), 1.68(6 \mathrm{H}, \mathrm{s}), 1.07-1.29(10 \mathrm{H}$, m), $1.19(3 \mathrm{H}, \mathrm{s}), 1.12(3 \mathrm{H}, \mathrm{s}), 1.10(3 \mathrm{H}, \mathrm{s}), 1.08(3 \mathrm{H}, \mathrm{s}), 0.94(3 \mathrm{H}, \mathrm{d}, J=6.8 \mathrm{~Hz}), 0.87(9 \mathrm{H}, \mathrm{s}), 0.83(3 \mathrm{H}, \mathrm{d}, J=6.4$ $\mathrm{Hz}), 0.06(6 \mathrm{H}, \mathrm{s}) ;{ }^{13} \mathrm{C}$ NMR $\left(75 \mathrm{MHz}, \mathrm{CDCl}_{3}\right) \delta 176.8,176.6,171.0,162.1,160.4,157.3,148.4,145.4,146.6$, $136.5,135.3,133.8,128.7,128.2,128.0,127.8,125.4,122.3,115.8,115.3,113.2,112.2,105.1,97.2,96.4,82.2$, $82.1,78.5,78.0,77.9,77.8,75.3,74.7,72.5,72.0,68.6,66.3,66.2,56.1,46.9,46.8,42.6,42.0,39.1,36.9,36.6$, $36.1,35.7,34.4,34.2,32.2,32.1,32.0,31.8,31.6,28.8,28.2,28.1,27.3,26.2,26.0,25.9,25.8,25.7,25.2,23.9$, $23.8,22.2,21.4,20.5,20.2,18.1,15.2,15.1,-4.5,-4.8$. MS (ES) $m / z(\%): 1541.45\left([\mathrm{MNa}]^{+}, 30\right)$.
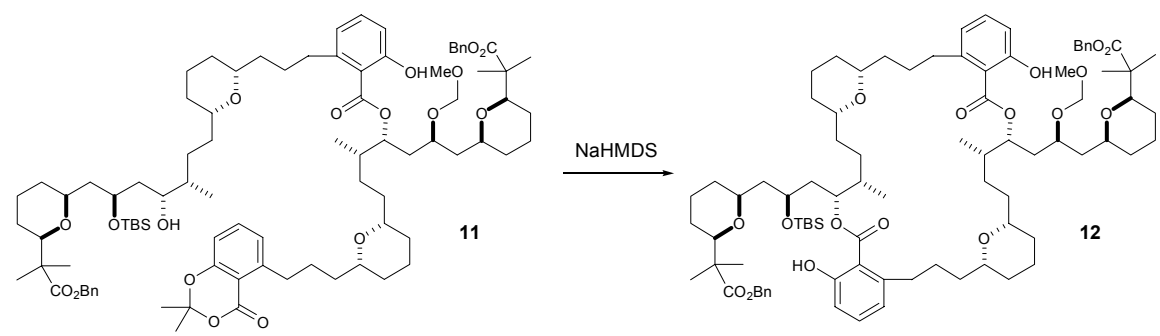

Macrocycle 12. To a stirred solution of alcohol $11(22.3 \mathrm{mg}, 0.0147 \mathrm{mmol})$ in THF $(2 \mathrm{~mL})$ at $-78{ }^{\circ} \mathrm{C}$ was added NaHMDS $(74 \mu \mathrm{L}, 0.074 \mathrm{mmol})$. The reaction was allowed to warm to -20 ${ }^{\circ} \mathrm{C}$ over the course of $1 \mathrm{~h}$ and kept at that temperature for $30 \mathrm{~min}$ before addition of $\mathrm{pH}=7$ phosphate buffer $(3$

$\mathrm{mL})$. The quenched reaction was then diluted with water $(20 \mathrm{~mL})$ and extracted with $\mathrm{Et}_{2} \mathrm{O}(3 \times 30 \mathrm{~mL})$. The combined organic phase was dried over $\mathrm{Na}_{2} \mathrm{SO}_{4}$, filtered, concentrated, and purified by $\mathrm{FC}(1 / 15 / 84$ $\left.\mathrm{Et}_{3} \mathrm{~N} / \mathrm{EtOAc} / \mathrm{Hex}\right)$ to afford $11 \mathrm{mg}(50 \%)$ of macrocycle 12. $[\alpha]_{\mathrm{D}}=+12.7\left(\mathrm{CHCl}_{3}, c\right.$ 0.70). IR (film) 3400,2933 , $2858,1732,1652,1606,1578,1450,1371,1311,1253,1214,1089,1047,916 \mathrm{~cm}^{-1} ;{ }^{1} \mathrm{H}$ NMR $\left(400 \mathrm{MHz}, \mathrm{CDCl}_{3}\right) \delta$ $11.35(1 \mathrm{H}, \mathrm{s}), 11.06(1 \mathrm{H}, \mathrm{s}), 7.27-7.38(10 \mathrm{H}, \mathrm{m}), 7.16-7.23(2 \mathrm{H}, \mathrm{m}), 6.78(2 \mathrm{H}, \mathrm{dd}, J=2.0,8.0 \mathrm{~Hz}), 6.61(1 \mathrm{H}, \mathrm{d}, J$ $=7.6 \mathrm{~Hz}), 6.56(1 \mathrm{H}, \mathrm{d}, J=7.6 \mathrm{~Hz}), 5.37-5.43(1 \mathrm{H}, \mathrm{m}), 5.30-5.35(1 \mathrm{H}, \mathrm{m}), 5.06(2 \mathrm{H}, \mathrm{s}), 5.04(2 \mathrm{H}, \mathrm{s}), 4.57(1 \mathrm{H}, \mathrm{d}, J$ $=6.8 \mathrm{~Hz}), 4.51(1 \mathrm{H}, \mathrm{d}, J=6.8 \mathrm{~Hz}), 3.84-3.91(1 \mathrm{H}, \mathrm{m}), 3.64-3.71(1 \mathrm{H}, \mathrm{m}), 3.41(1 \mathrm{H}, \mathrm{d}, J=10.8 \mathrm{~Hz}), 3.35(1 \mathrm{H}, \mathrm{d}, J$ $=11.2 \mathrm{~Hz}), 3.30(3 \mathrm{H}, \mathrm{s}), 3.15-3.23(4 \mathrm{H}, \mathrm{m}), 3.06-3.13(4 \mathrm{H}, \mathrm{dd}, J=4.4,6.8 \mathrm{~Hz}), 2.46-2.58(2 \mathrm{H}, \mathrm{m}), 1.36-1.94$ $(43 \mathrm{H}, \mathrm{m}), 0.94-1.29(22 \mathrm{H}, \mathrm{m}), 0.86-0.87(3 \mathrm{H}, \mathrm{m}), 0.85(9 \mathrm{H}, \mathrm{s}),-0.01(6 \mathrm{H}, \mathrm{s}) ;{ }^{13} \mathrm{C} \mathrm{NMR}\left(75 \mathrm{MHz}, \mathrm{CDCl}_{3}\right) \delta 176.6$, $176.5,171.6,171.3,162.8,162.3,145.8,145.6,136.6,133.9,133.8,128.7,128.2,128.0,122.8,115.8,112.9$, $112.5,96.5,82.1,81.8,78.1,77.2,75.0,74.9,72.6,66.5,66.2,66.1,56.0,53.6,46.8,46.4,45.6,42.1,39.0,37.1$, $37.0,36.9,36.8,36.7,33.9,32.2,32.0,31.5,31.4,29.2,29.0,28.3,28.2,26.1,25.9,25.7,23.9,23.8,22.7,22.3$, 22.0, 19.4, 18.2, 15.9, 15.7, -3.9, -4.7. MS (ES) $m / z(\%): 1482.95\left([\mathrm{MNa}]^{+}, 100\right)$.

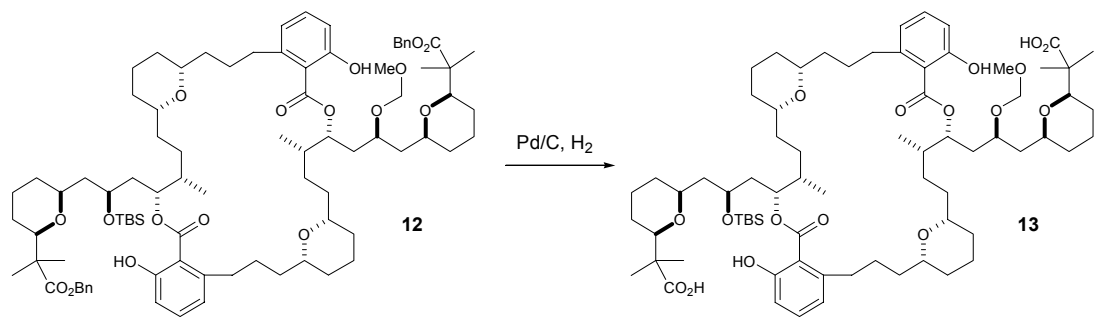

Bis-Acid 13. To a stirred solution of bisbenzyl ester 12 (11 $\mathrm{mg}, 0.0075 \mathrm{mmol})$ in $\mathrm{MeOH}(2 \mathrm{~mL})$ at ambient temperature was quickly added $10 \% \mathrm{Pd} / \mathrm{C}(\sim 1-2 \mathrm{mg})$. The reaction flask was then sealed with a three way stopper fitted with a $\mathrm{H}_{2}$ balloon. The flask was placed under 
house vacuum and flushed with $\mathrm{H}_{2}$ then placed under vacuum once again. This process was repeated $(6 \times)$. The reaction was then allowed to stir under balloon pressure of $\mathrm{H}_{2}$ for $3.5 \mathrm{~h}$. The reaction was filtered through a short pad of celite, and the catalyst was washed extensively with $\mathrm{CH}_{2} \mathrm{Cl}_{2}$ and $\mathrm{MeOH}$. After concentration, and purification by $\mathrm{FC}\left(1 / 2 / 97 \mathrm{AcOH} / \mathrm{MeOH} / \mathrm{CH}_{2} \mathrm{Cl}_{2}\right) 9.0 \mathrm{mg}(93 \%)$ of bis-acid 13 was isolated. $[\alpha]_{\mathrm{D}}=+19.3\left(\mathrm{CHCl}_{3}, c\right.$ 0.61). IR (film) 3300, 2934, 2859, 1705, 1652, 1606, 1450, 1369, 1295, 1252, 1214, 1089, 1047, $913 \mathrm{~cm}^{-1} ;{ }^{1} \mathrm{H}$ NMR $\left(400 \mathrm{MHz}, \mathrm{CDCl}_{3}\right) \delta 7.25(2 \mathrm{H}, \mathrm{t}, J=8.0 \mathrm{~Hz}), 6.79-6.84(2 \mathrm{H}, \mathrm{m}), 6.65-6.70(2 \mathrm{H}, \mathrm{m}), 5.33-5.40(2 \mathrm{H}, \mathrm{m}), 4.61$ $(1 \mathrm{H}, \mathrm{d}, J=6.8 \mathrm{~Hz}), 4.57(1 \mathrm{H}, \mathrm{d}, J=6.8 \mathrm{~Hz}), 3.85-3.92(1 \mathrm{H}, \mathrm{m}), 3.64-3.72(1 \mathrm{H}, \mathrm{m}), 3.43-3.53(2 \mathrm{H}, \mathrm{m}), 3.34-3.36$ $(2 \mathrm{H}, \mathrm{m}), 3.37(3 \mathrm{H}, \mathrm{s}), 3.05-3.23(6 \mathrm{H}, \mathrm{m}), 2.53-2.63(2 \mathrm{H}, \mathrm{m}), 1.66-1.94(14 \mathrm{H}, \mathrm{m}), 1.38-1.64(24 \mathrm{H}, \mathrm{m}), 1.12-1.26$ $(12 \mathrm{H}, \mathrm{m}), 1.25(3 \mathrm{H}, \mathrm{s}), 1.09(3 \mathrm{H}, \mathrm{s}), 1.07(3 \mathrm{H}, \mathrm{s}), 0.96-1.02(6 \mathrm{H}, \mathrm{m}), 0.88(3 \mathrm{H}, \mathrm{s}), 0.86(9 \mathrm{H}, \mathrm{s}), 0.01(3 \mathrm{H}, \mathrm{s}),-0.01$ $(3 \mathrm{H}, \mathrm{s}) ;{ }^{13} \mathrm{C}$ NMR $\left(75 \mathrm{MHz}, \mathrm{CDCl}_{3}\right) \delta 178.9,178.8,171.4,171.3,162.8,162.4,145.8,145.6,134.0,122.8,116.0$, $112.8,112.5,96.9,82.7,82.6,78.6,78.2,78.1,77.4,75.3,75.1,73.1,66.8,56.0,46.5,45.1,42.1,39.3,37.3,37.1$, $37.0,36.8,33.8,33.7,32.2,32.0,31.5,29.9,29.8,29.2,29.1,28.4,28.3,26.1,25.5,23.9,23.7,23.6,23.2,23.0$, $18.2,16.2,16.0,-3.9,-4.7$. MS (ES) $m / z(\%): 1301.75\left([\mathrm{MNa}]^{+}, 80\right)$.

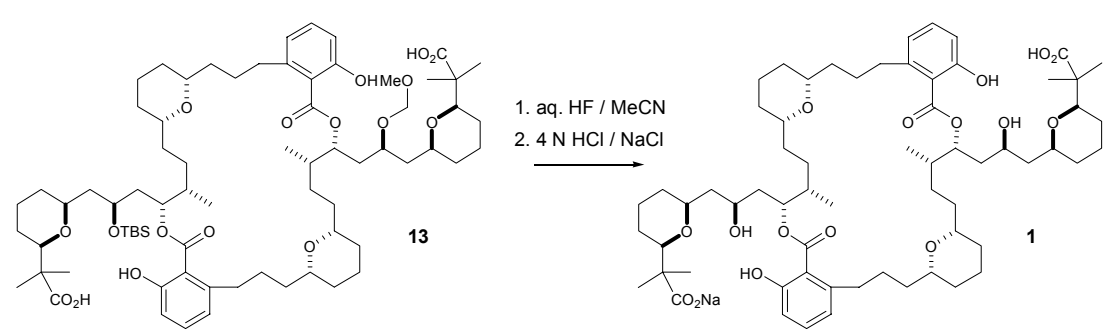

SCH 351448 (1). To a stirred solution of bis-acid 13 (6.5 $\mathrm{mg}, 0.005 \mathrm{mmol})$ in $\mathrm{CH}_{3} \mathrm{CN} \quad(0.3 \mathrm{~mL})$, was added $48 \%$ aqueous HF (0.030 $\mathrm{mL}, 3.13 \mathrm{mmol})$. The reaction was allowed to stir at ambient temperature in a plastic vial for $48 \mathrm{~h}$. The reaction was then diluted with hexane $(5$ $\mathrm{mL})$ and extracted with hexane $(3 \times 5$

$\mathrm{mL}$ ) from $4 \mathrm{~N}$ aq. $\mathrm{HCl}$ (saturated with $\mathrm{NaCl}, 2 \mathrm{~mL}$ ). The combined organic phase was dried over $\mathrm{Na}_{2} \mathrm{SO}_{4}$, filtered, and concentrated to afford $4.4 \mathrm{mg}(77 \%)$ of crude 1 as a fine white powder. Crude 1 contained detectable amounts of a byproduct by ${ }^{1} \mathrm{H}$ NMR which is also present in the natural isolated 1 . Purification by reversed phase HPLC (C18 column, 8/92 $\left.\mathrm{H}_{2} \mathrm{O} / \mathrm{MeOH}\right)$ provided pure 1. $[\alpha]_{\mathrm{D}}=+22.4\left(\mathrm{CHCl}_{3}, c\right.$ 0.10). IR (film) 3445, 2930, 2857, 1704, $1668,1605,1463,1295,1086,1047,757 \mathrm{~cm}^{-1} ;{ }^{1} \mathrm{H}$ NMR $\left(400 \mathrm{MHz}, \mathrm{CD}_{2} \mathrm{Cl}_{2}\right) \delta 7.25(2 \mathrm{H}, \mathrm{t}, J=8.0 \mathrm{~Hz}), 6.81(2 \mathrm{H}, \mathrm{d}$, $J=8.0 \mathrm{~Hz}), 6.72(2 \mathrm{H}, \mathrm{d}, J=7.2 \mathrm{~Hz}), 5.61-5.66(2 \mathrm{H}, \mathrm{m}), 3.71-3.77(2 \mathrm{H}, \mathrm{m}), 3.56-3.63(2 \mathrm{H}, \mathrm{m}), 3.50(2 \mathrm{H}, \mathrm{d}, J=$ $10.4 \mathrm{~Hz}), 3.09-3.19(6 \mathrm{H}, \mathrm{m}), 2.54(2 \mathrm{H}, \mathrm{td}, J=4.6,12.4 \mathrm{~Hz}), 2.20-2.10(2 \mathrm{H}, \mathrm{m}), 1.82-1.88(6 \mathrm{H}, \mathrm{m}), 1.57-1.79$ $(10 \mathrm{H}, \mathrm{m}), 1.38-1.60(22 \mathrm{H}, \mathrm{m}), 1.14-1.29(10 \mathrm{H}, \mathrm{m}), 1.13(6 \mathrm{H}, \mathrm{s}), 1.10(6 \mathrm{H}, \mathrm{s}), 1.01(6 \mathrm{H}, \mathrm{d}, J=6.4 \mathrm{~Hz}) ;{ }^{13} \mathrm{C}$ NMR $\left(75 \mathrm{MHz}, \mathrm{CD}_{2} \mathrm{Cl}_{2}\right) \delta 178.7,171.2,160.2,145.2,133.6,122.5,116.0,115.8,83.4,79.3,78.5,78.3,77.8,67.6,46.6$, $43.8,37.9,37.6,37.0,36.8,35.3,33.1,32.7,32.1,30.3,29.7,25.3,24.5,23.7,23.4,19.4,15.2$. MS (ES) $\mathrm{m} / z(\%)$ : $1143.65\left([\mathrm{MH}]^{+}, 100\right)$. 


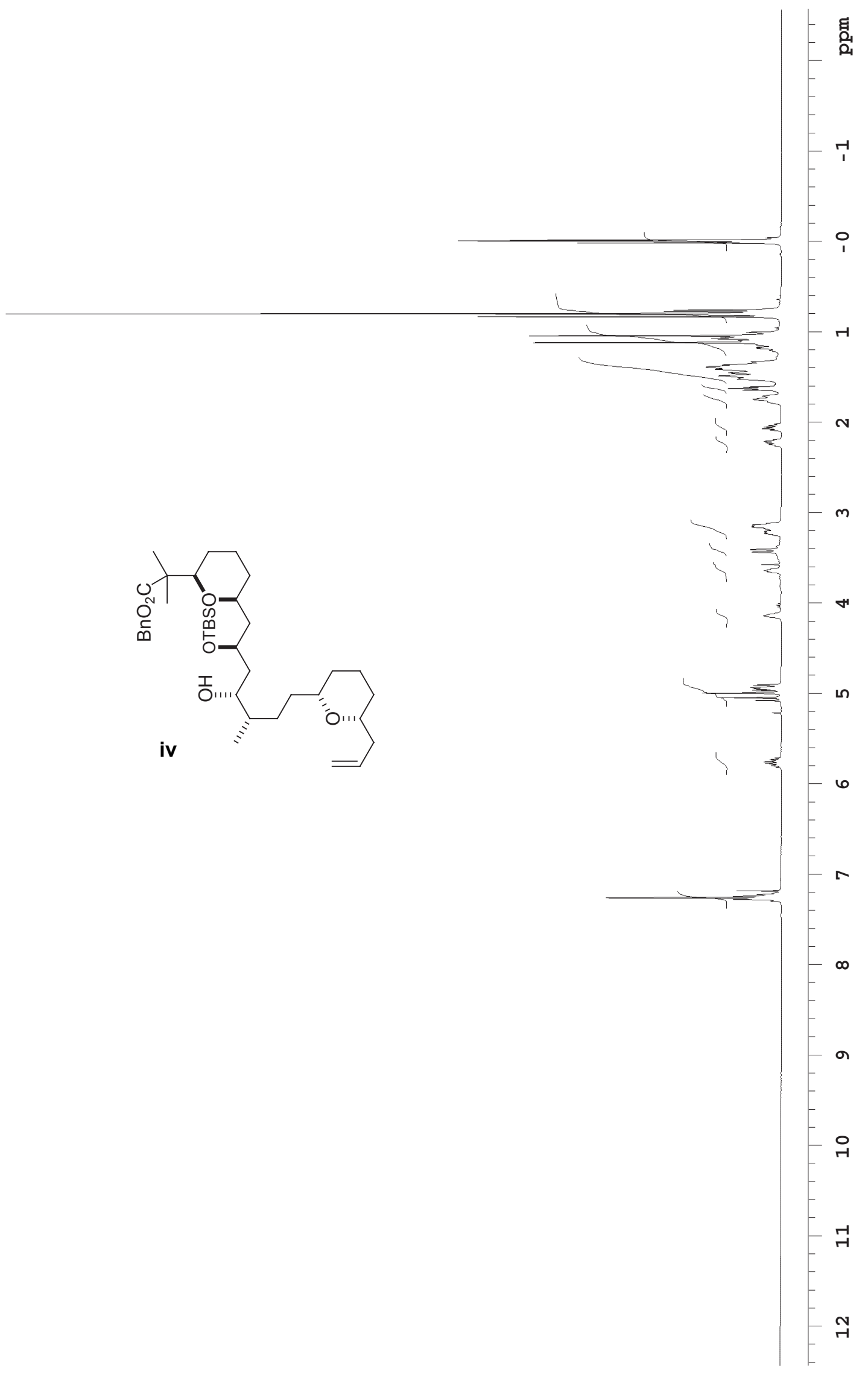




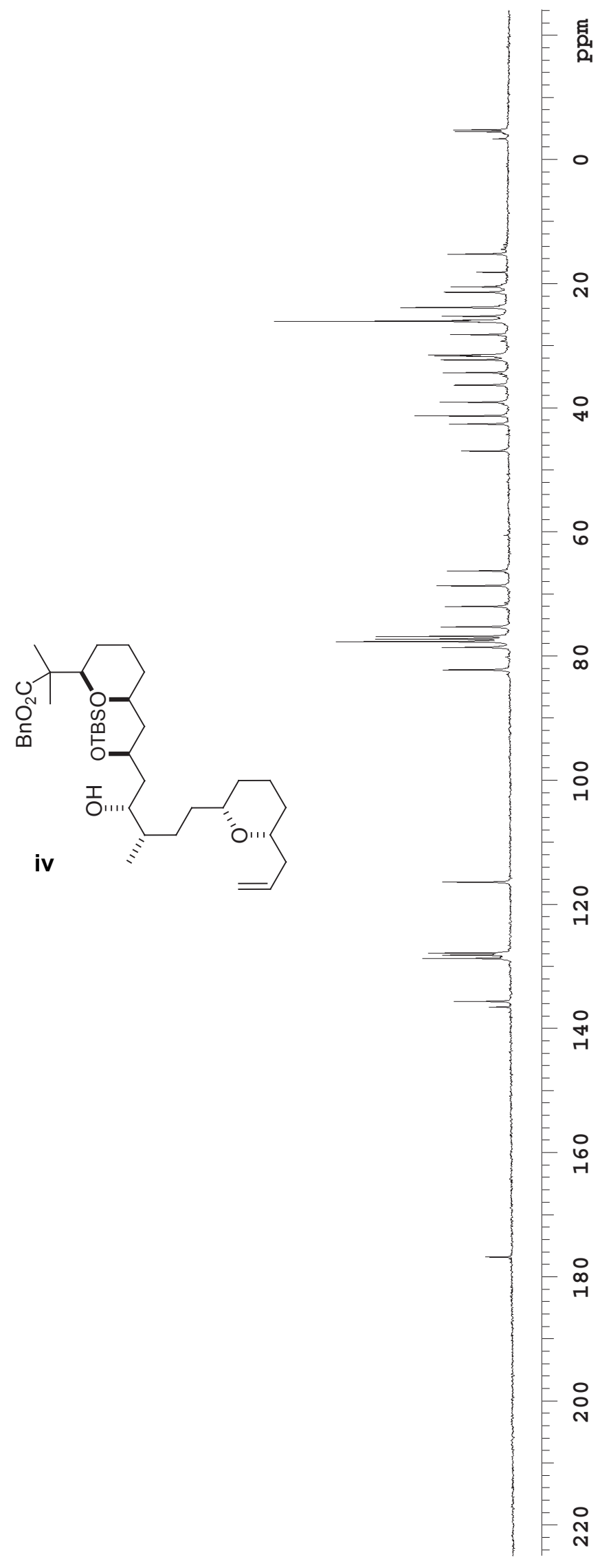




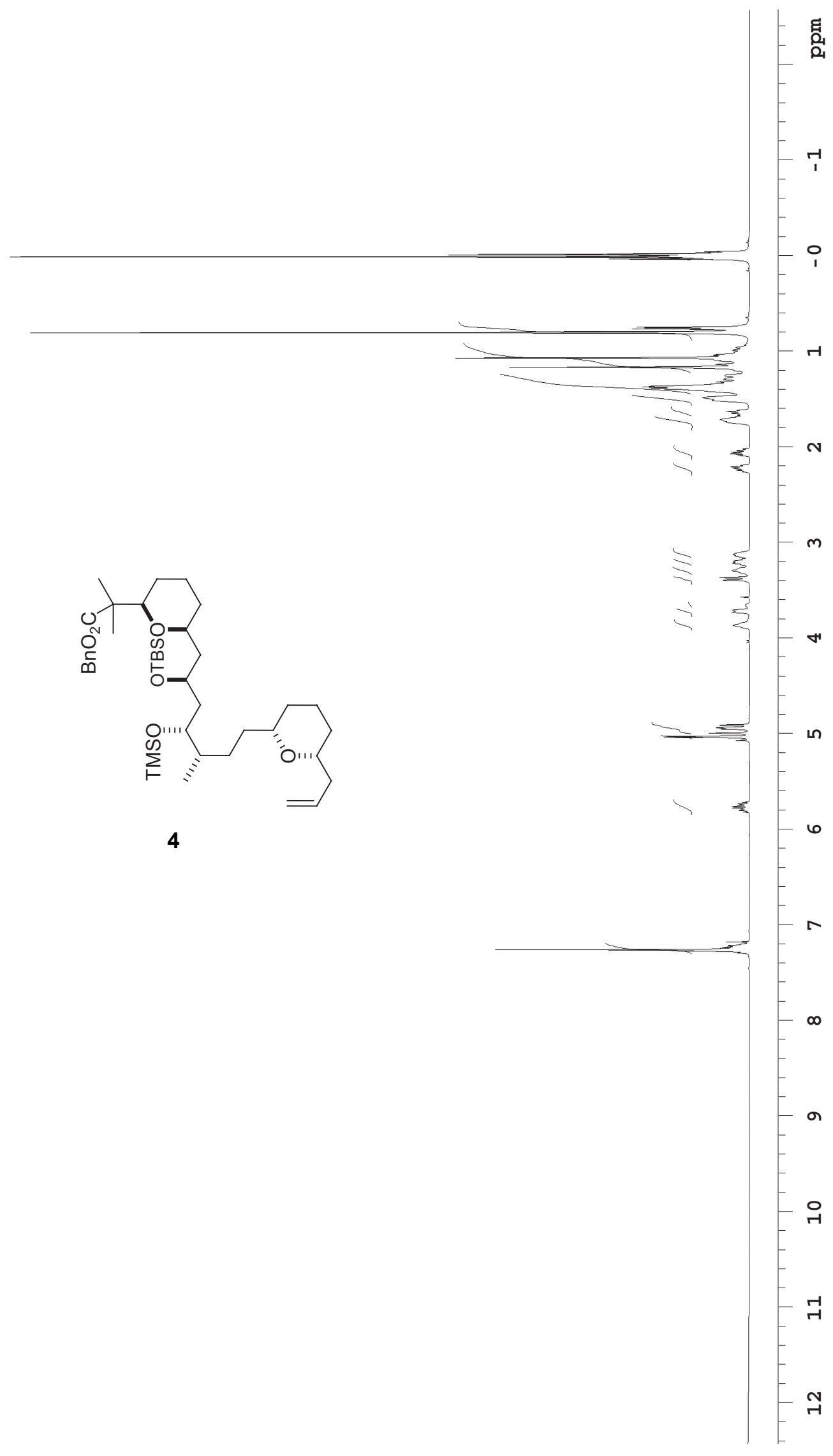




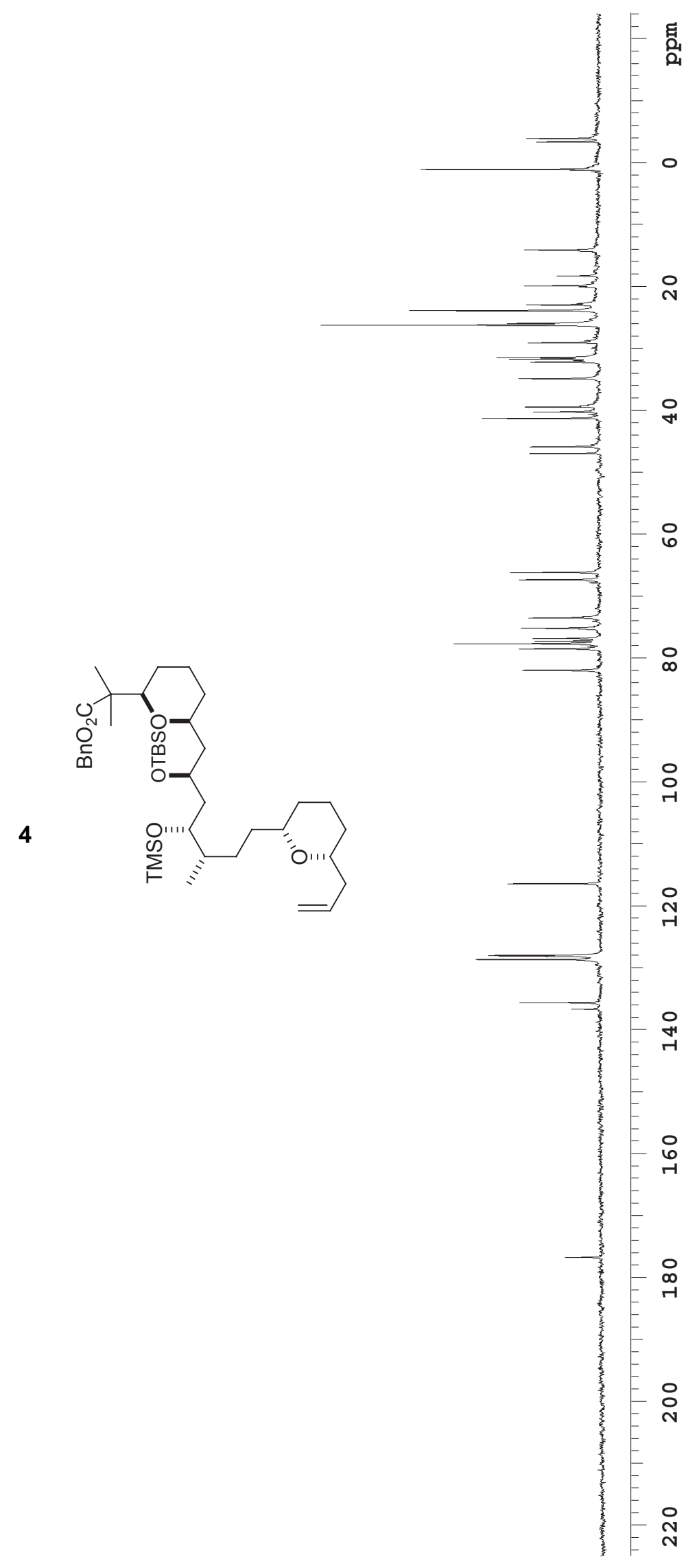




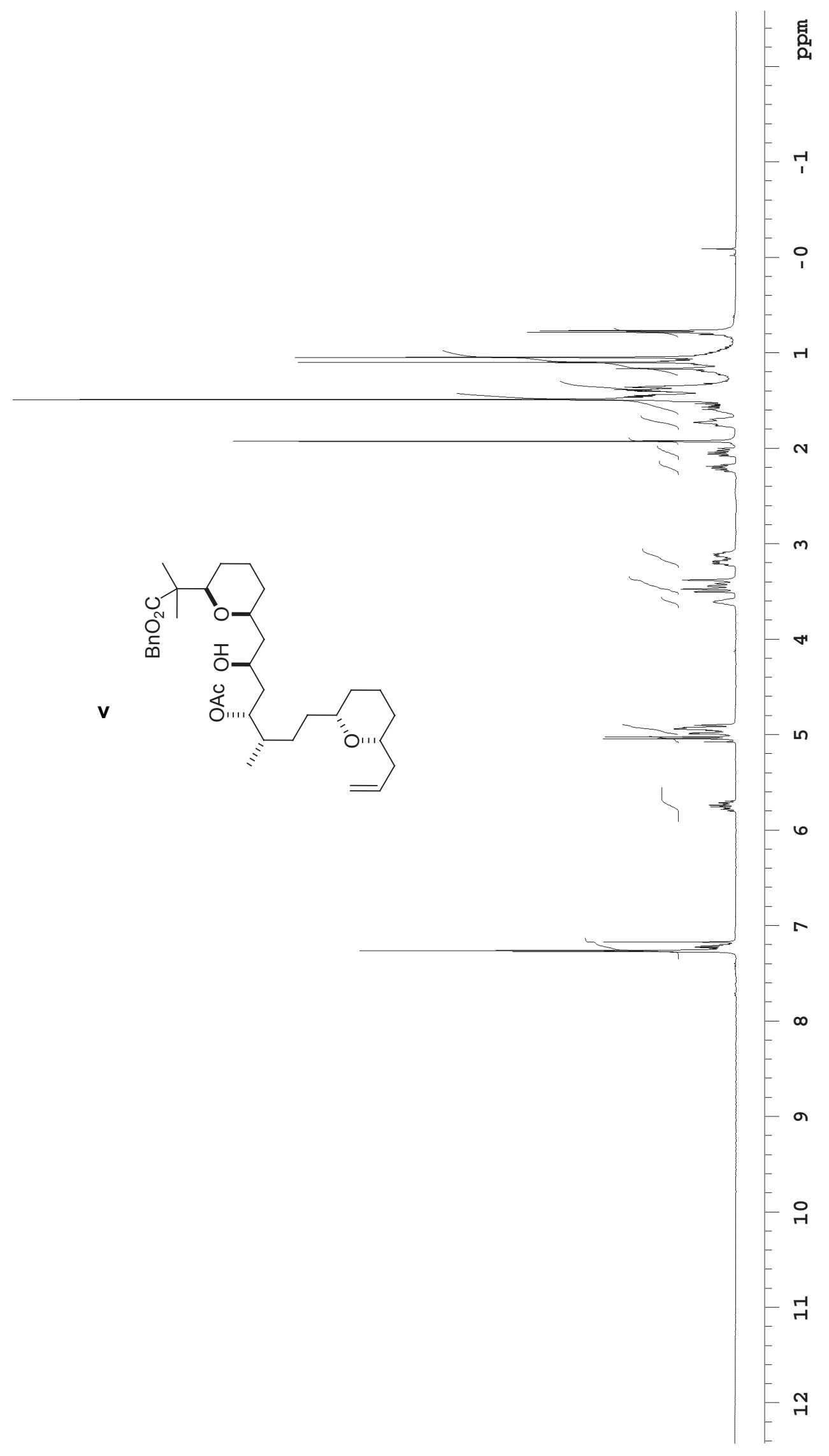




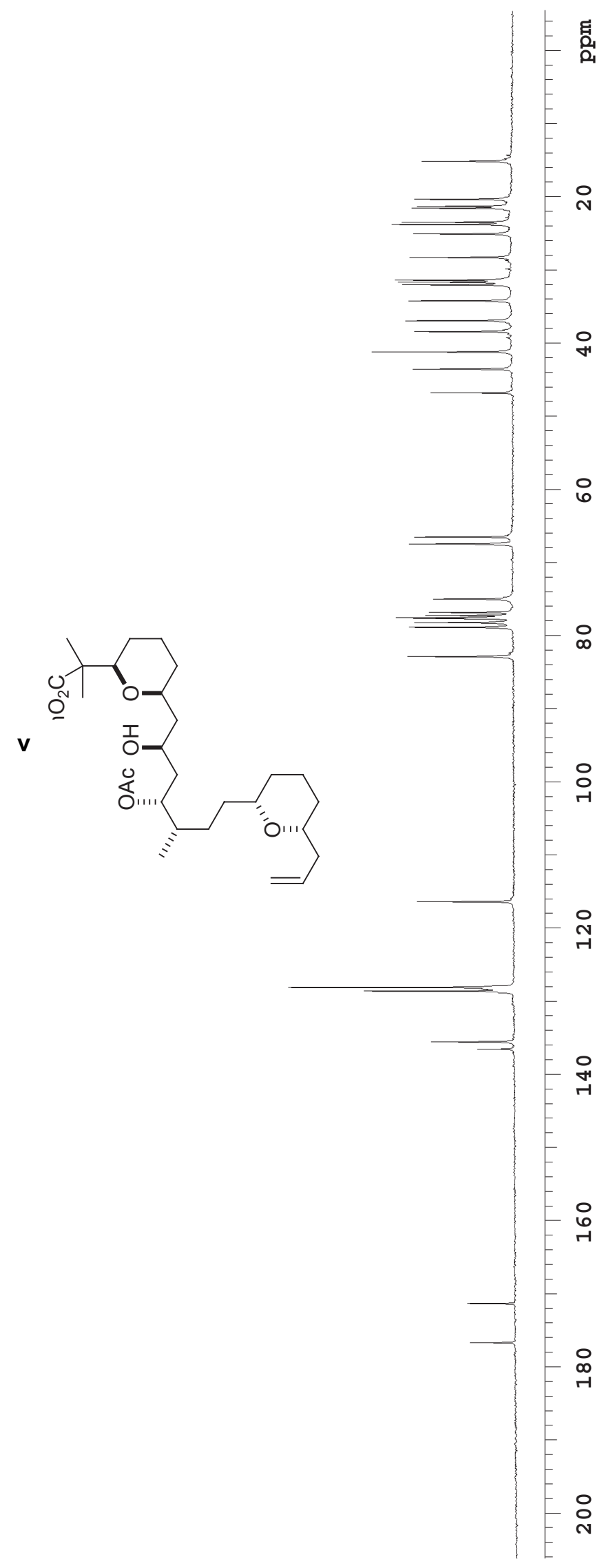




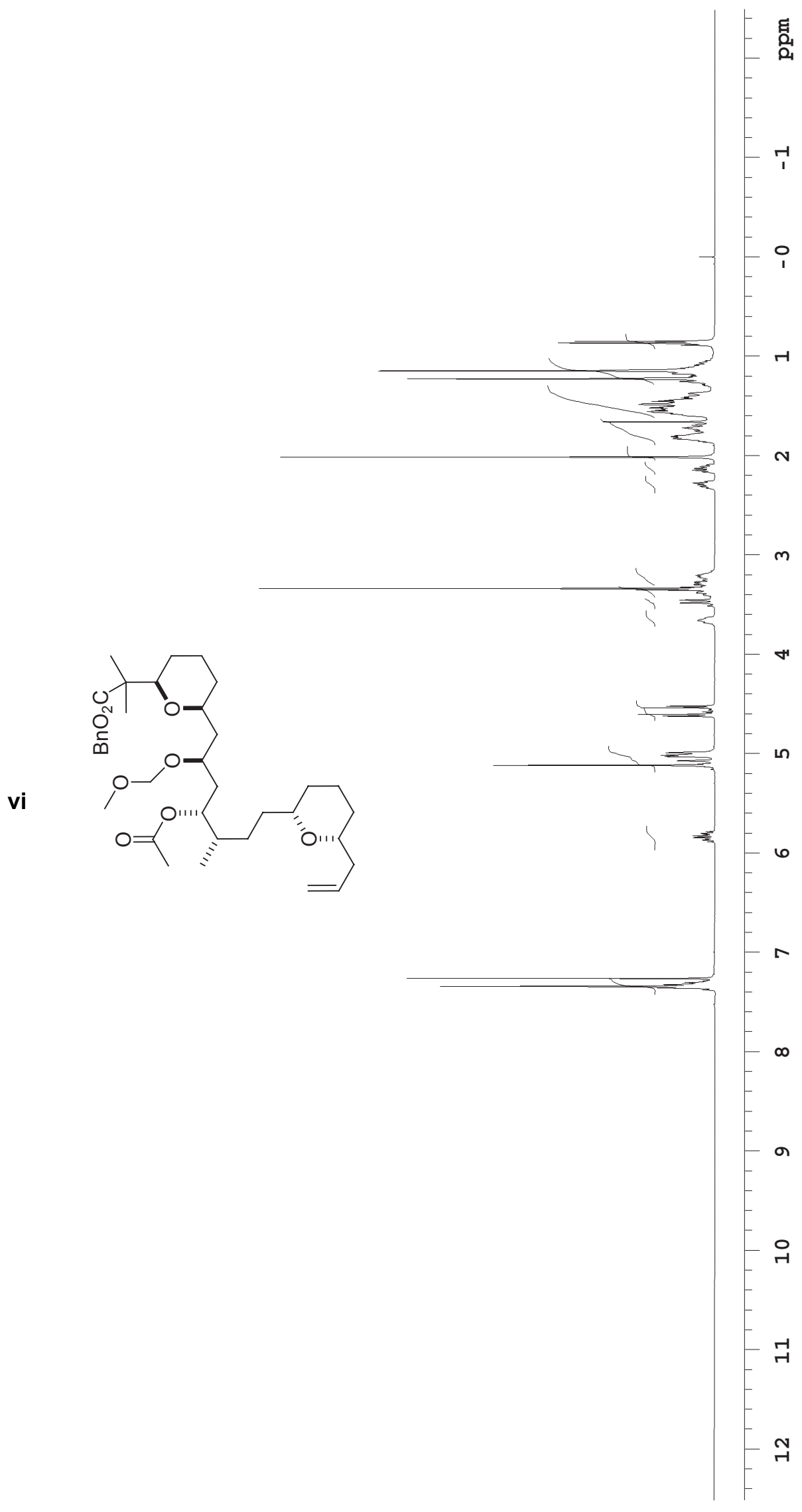




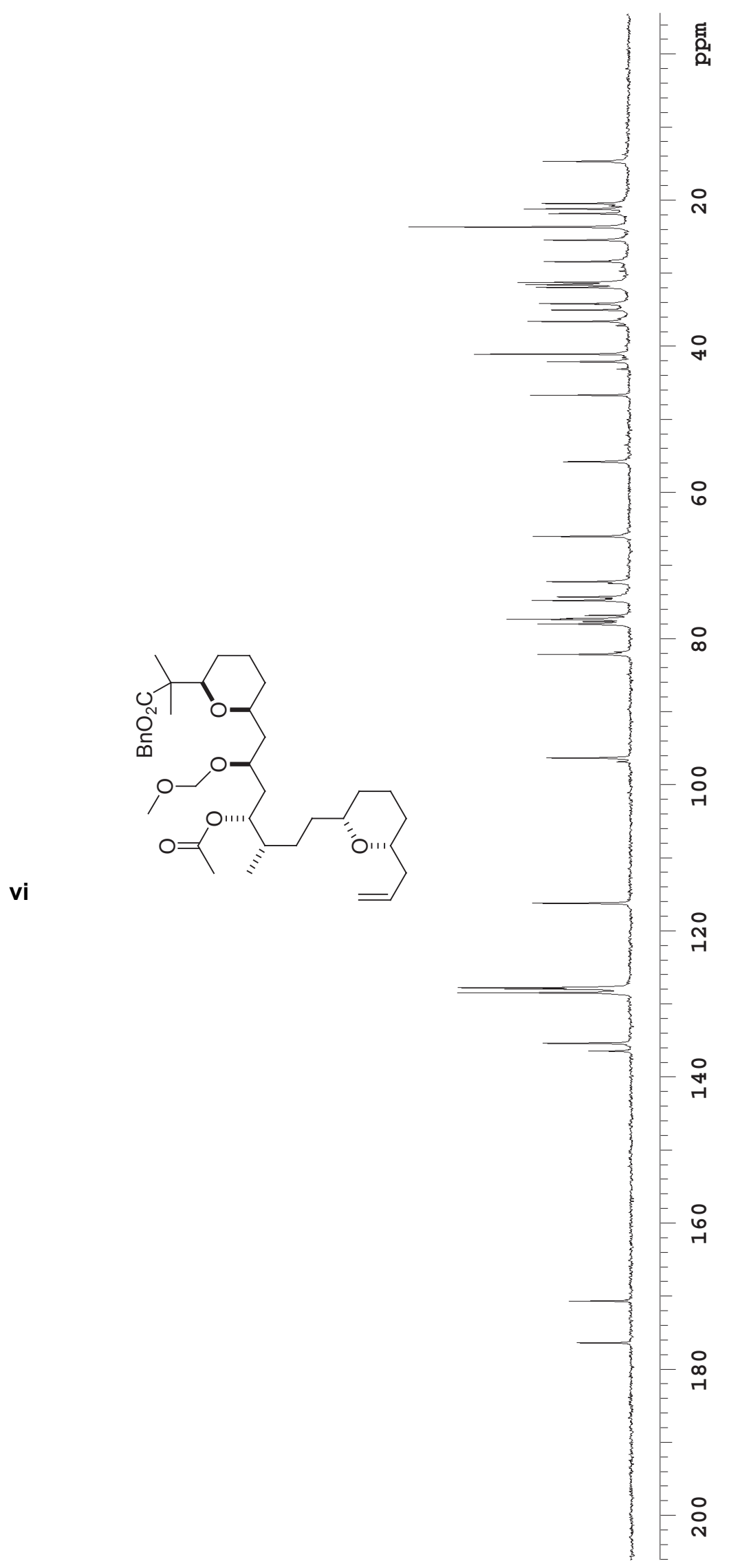




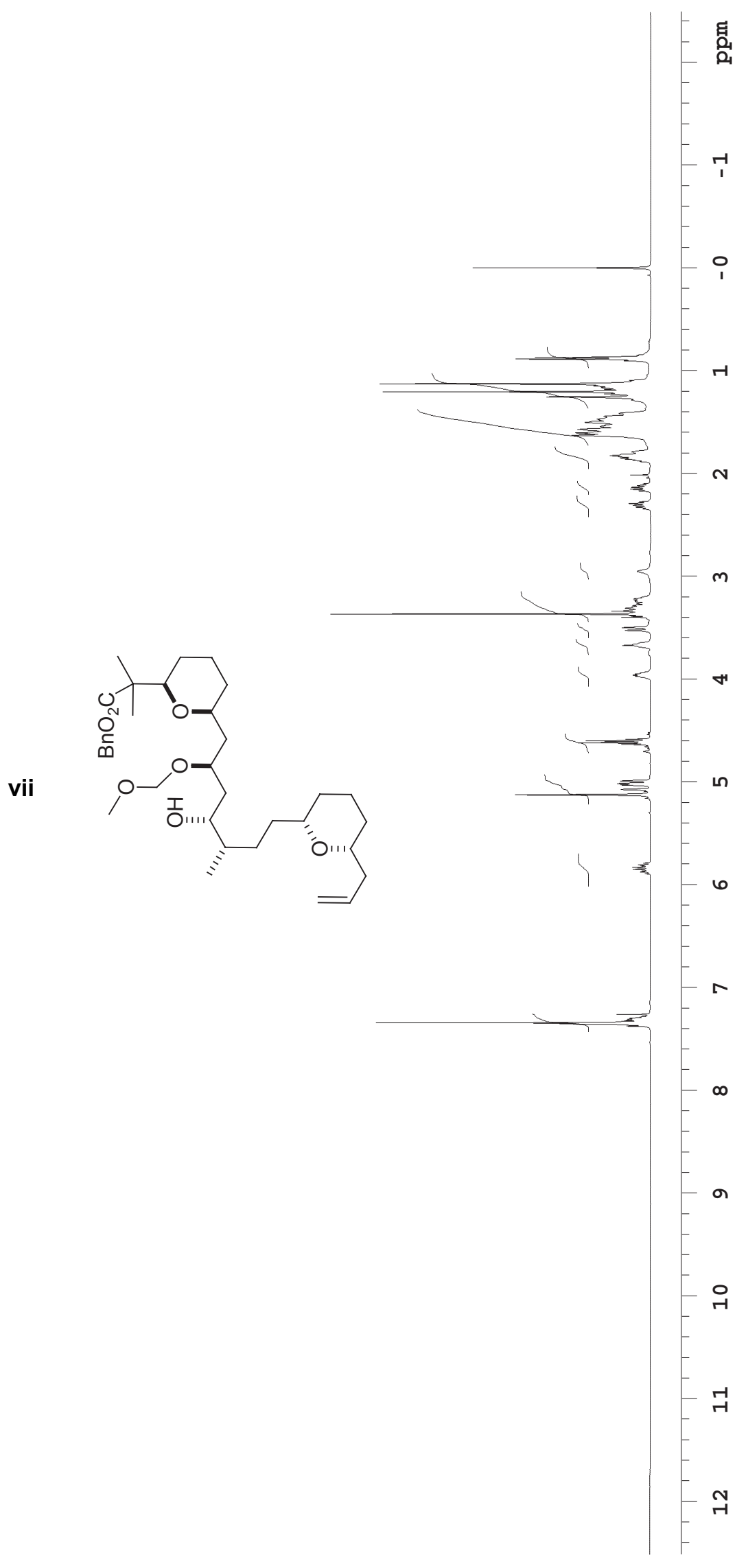




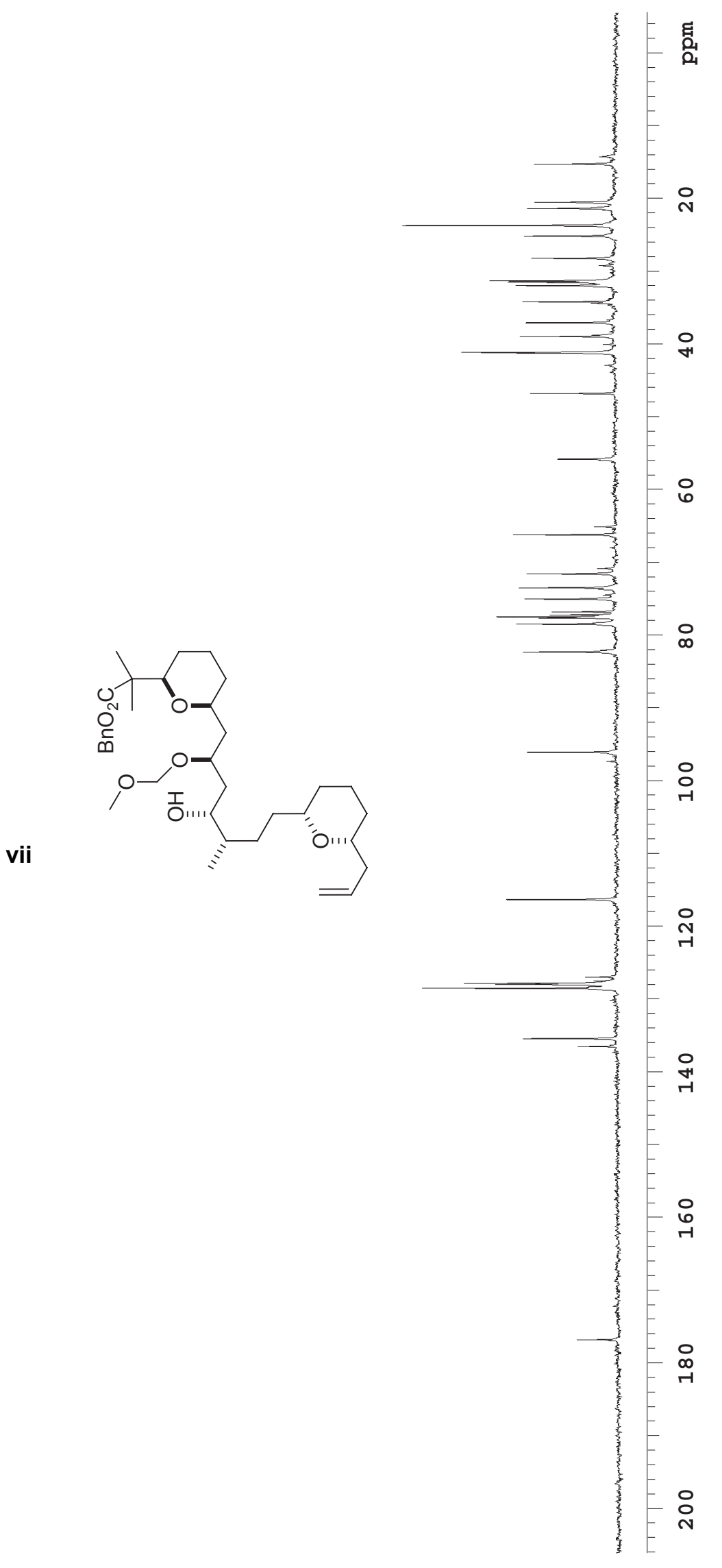




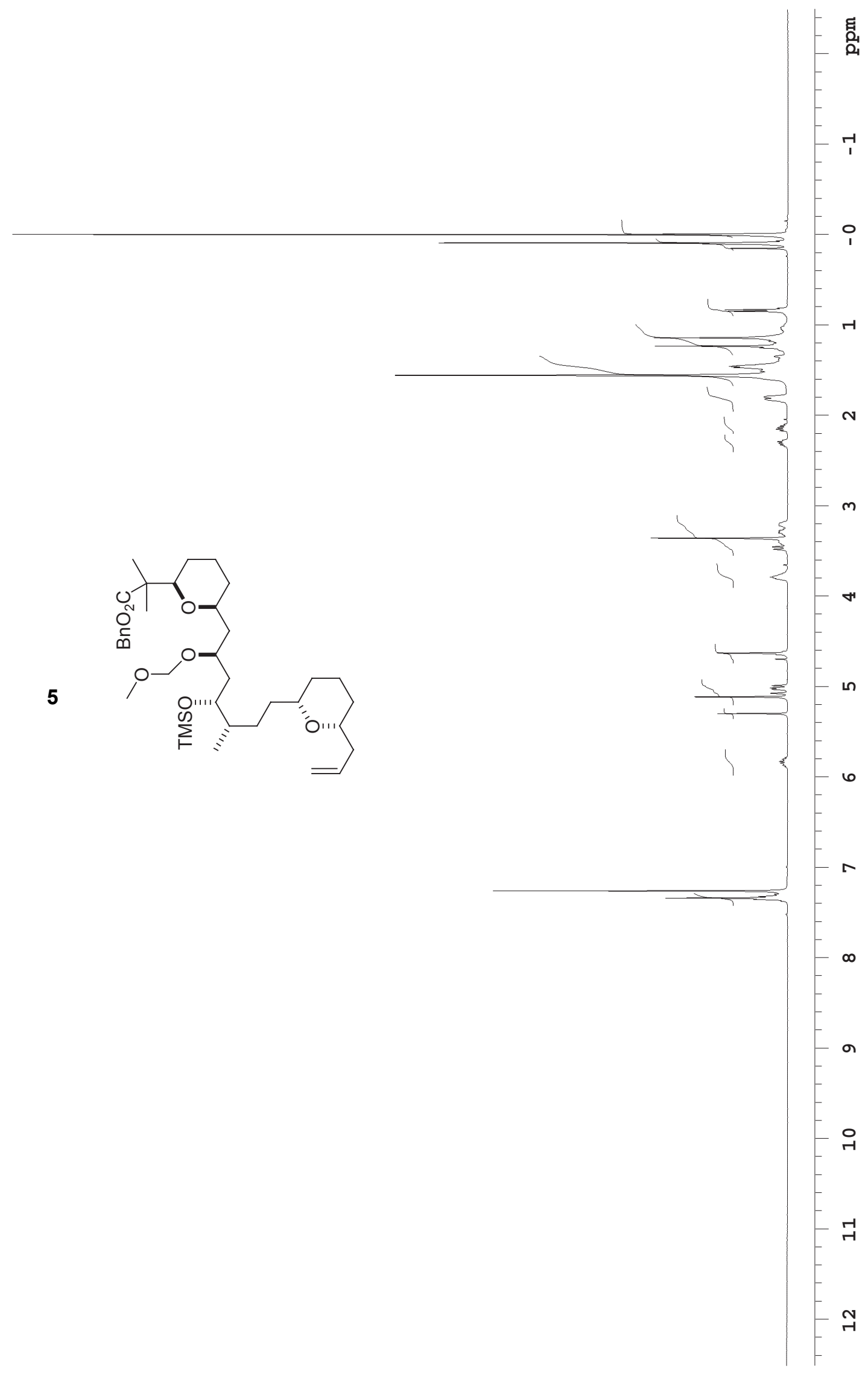




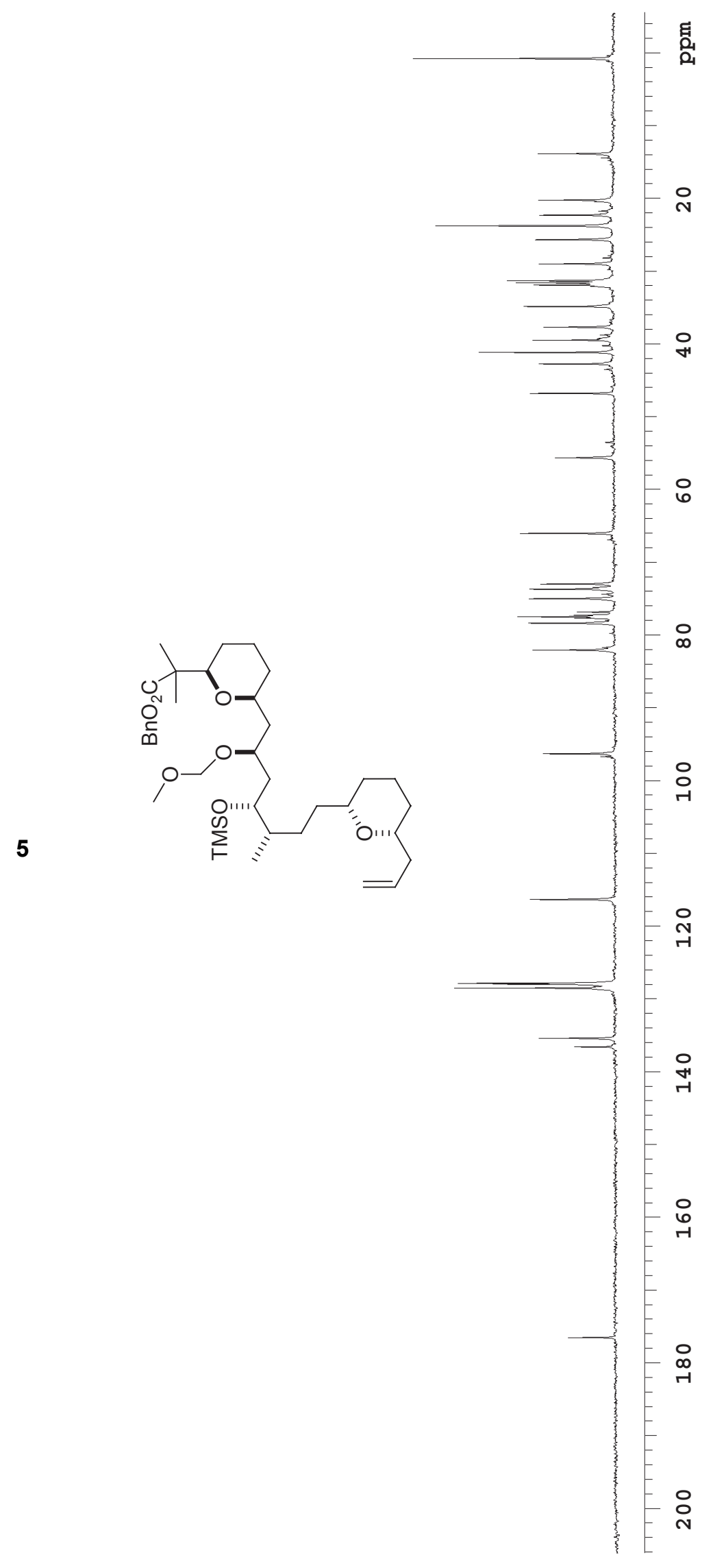




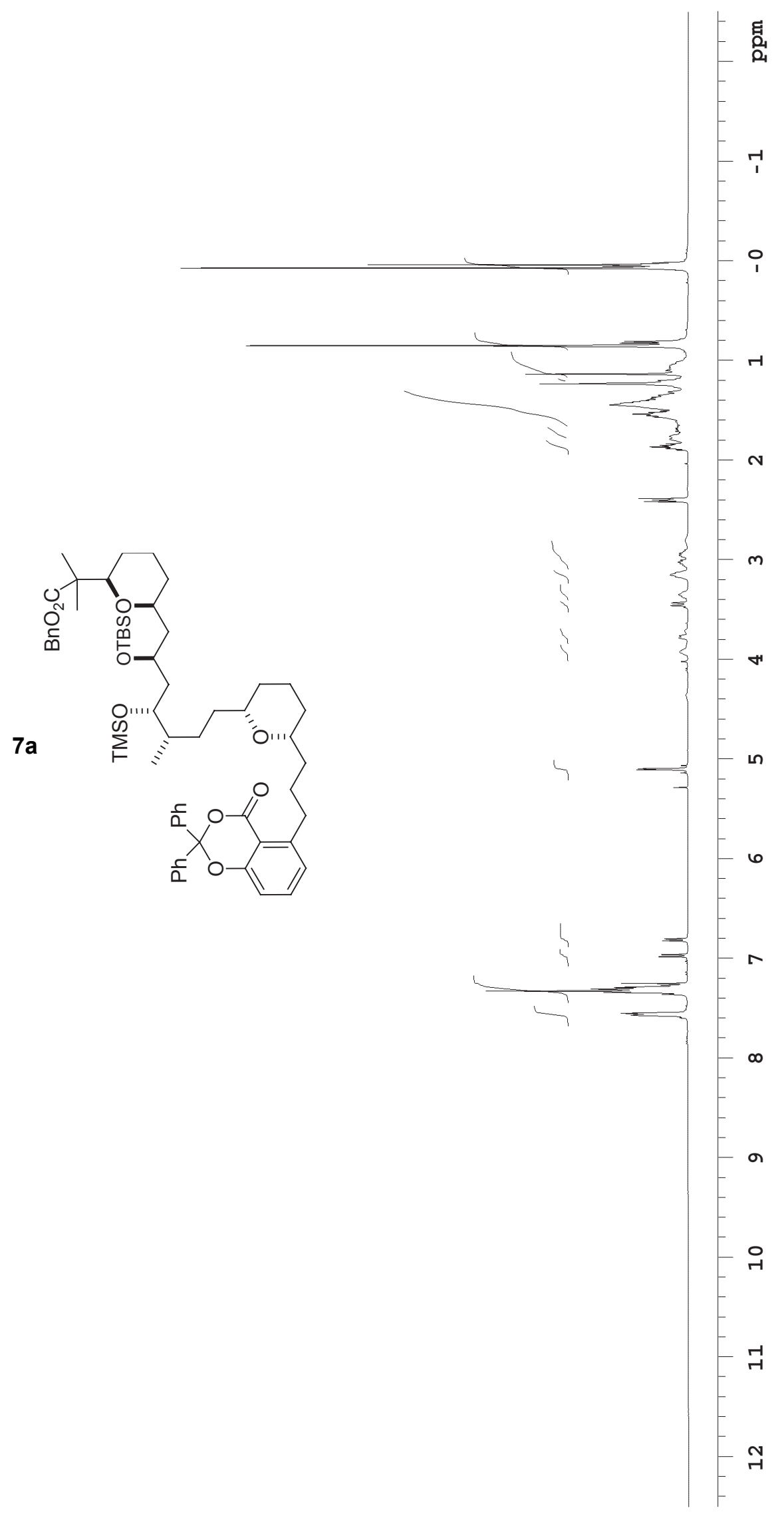




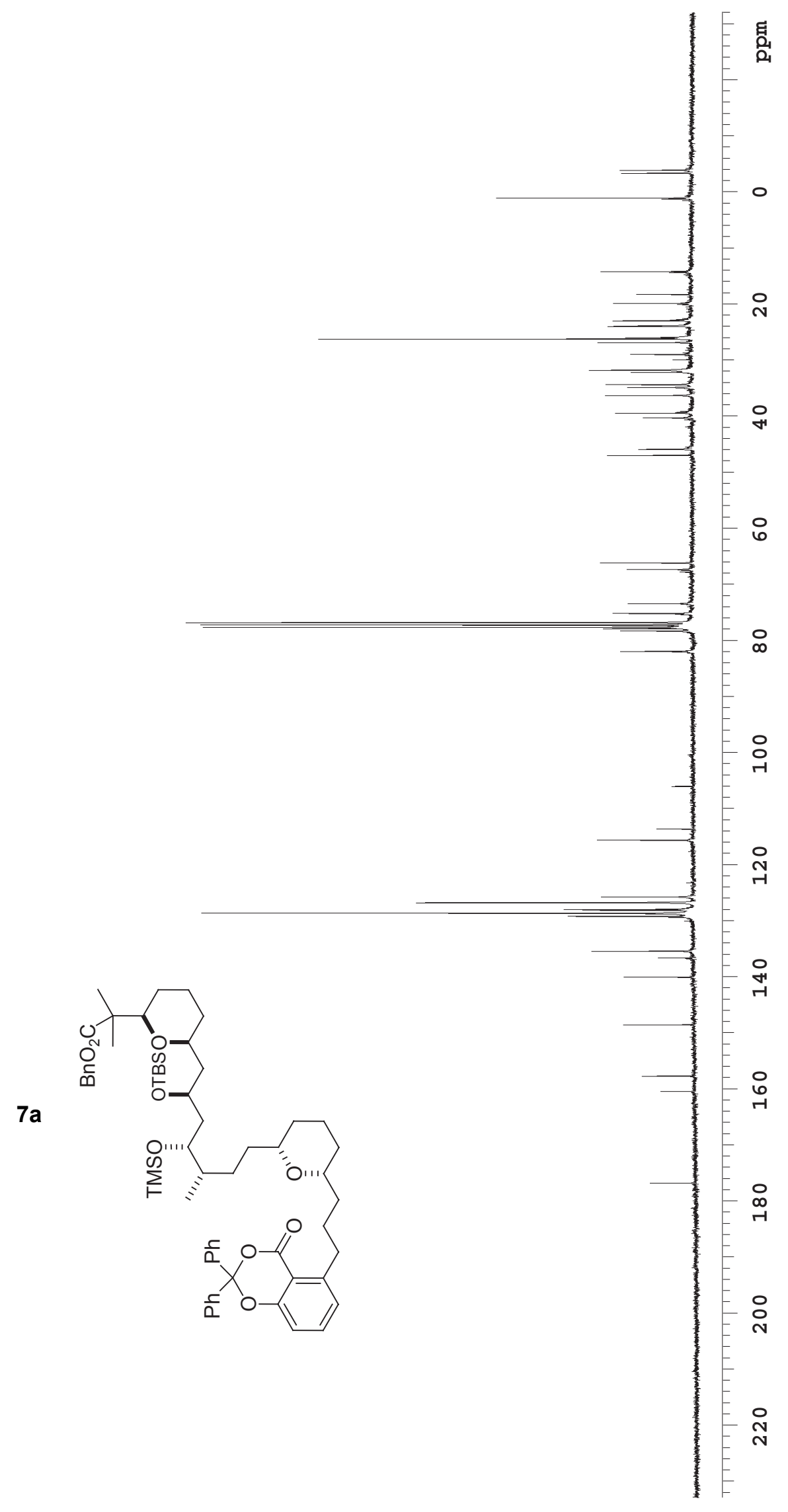




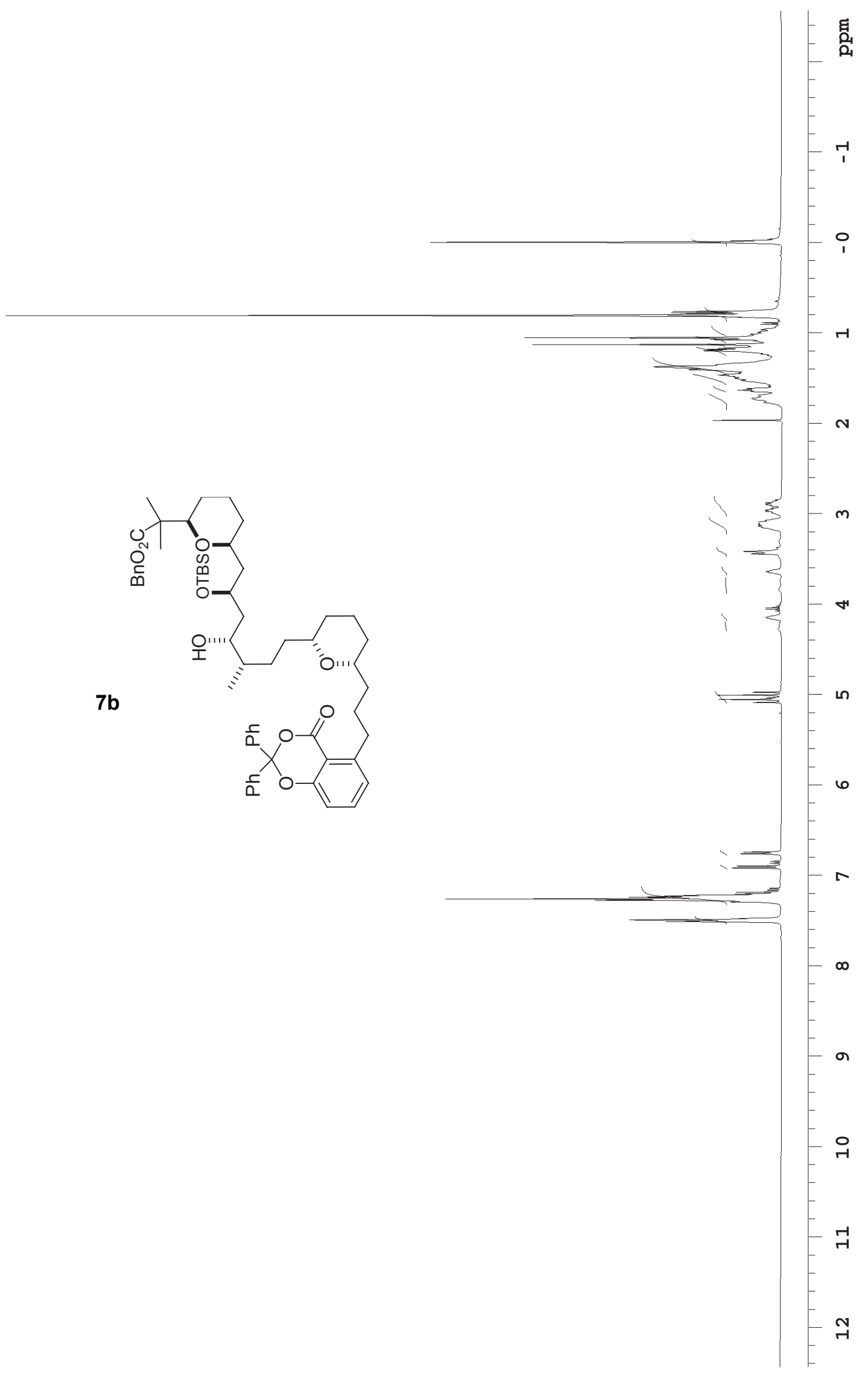




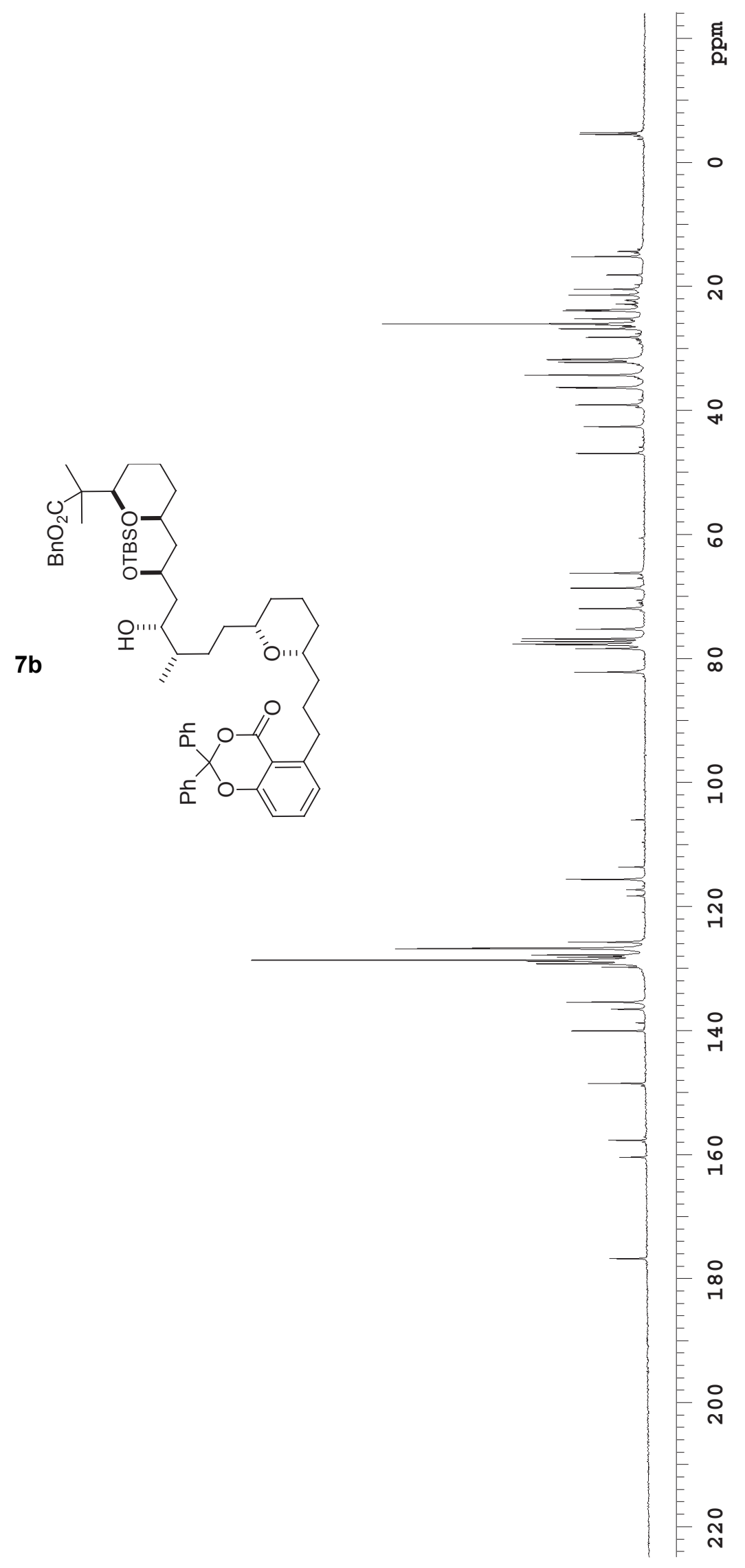




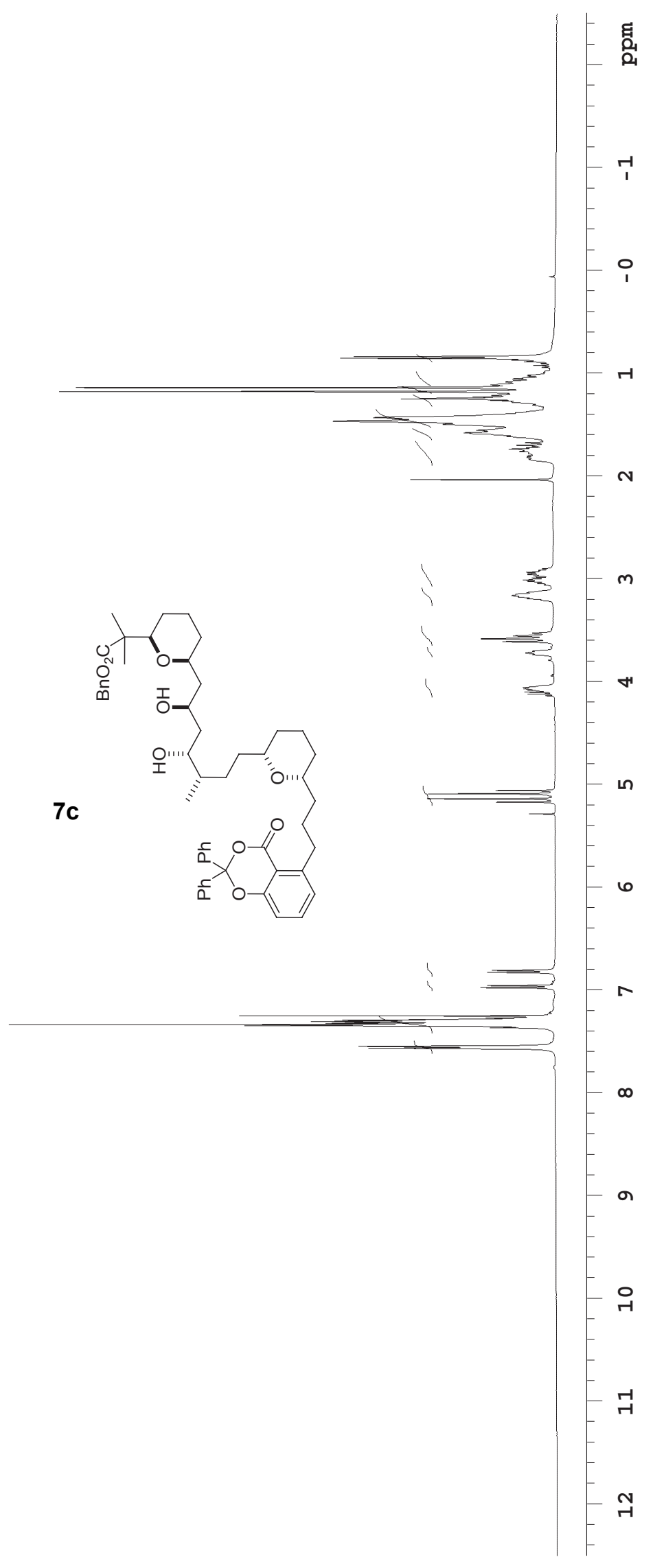


7c

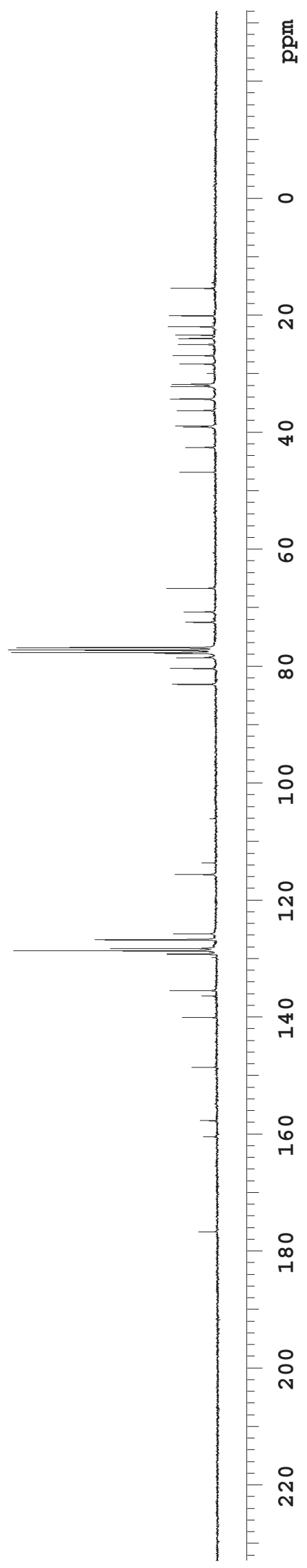

S28 


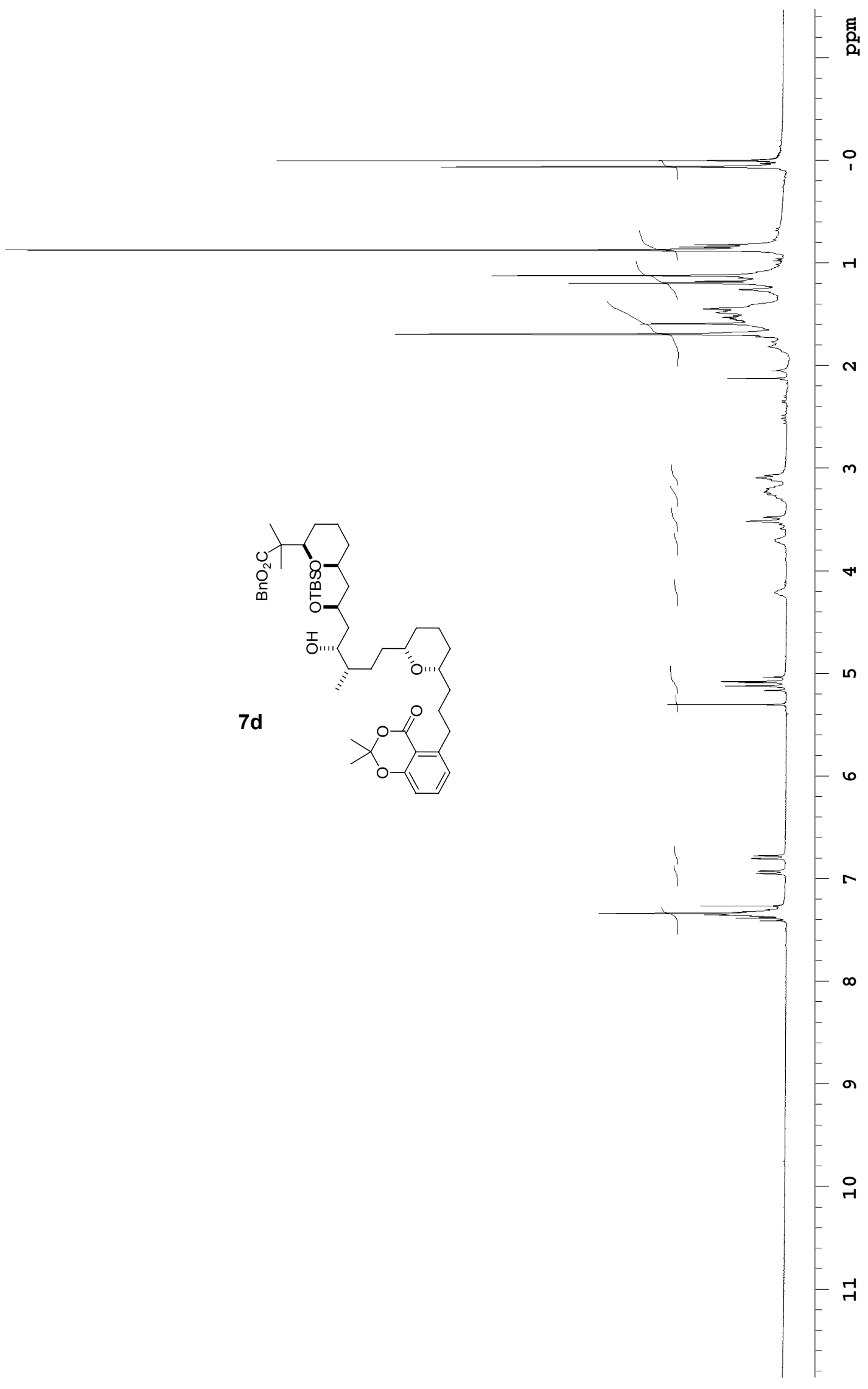




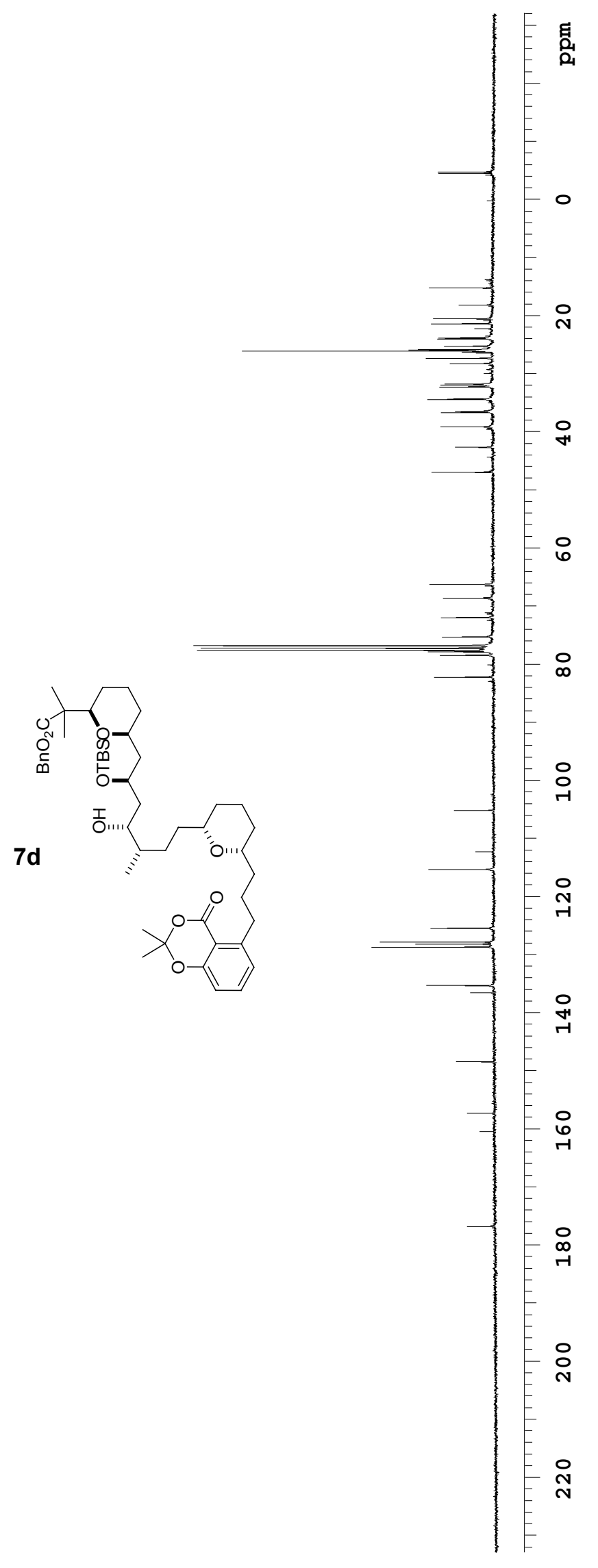




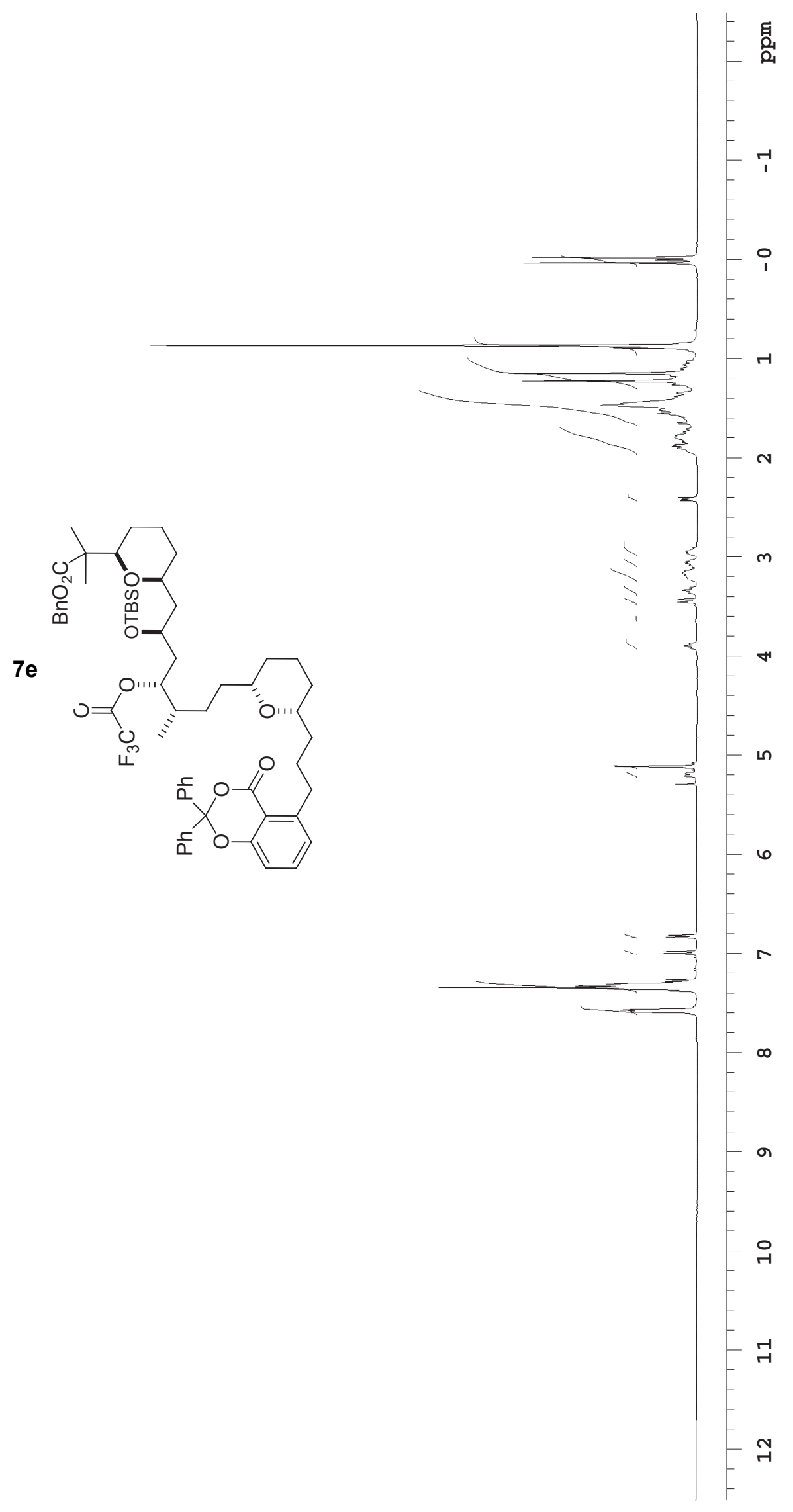




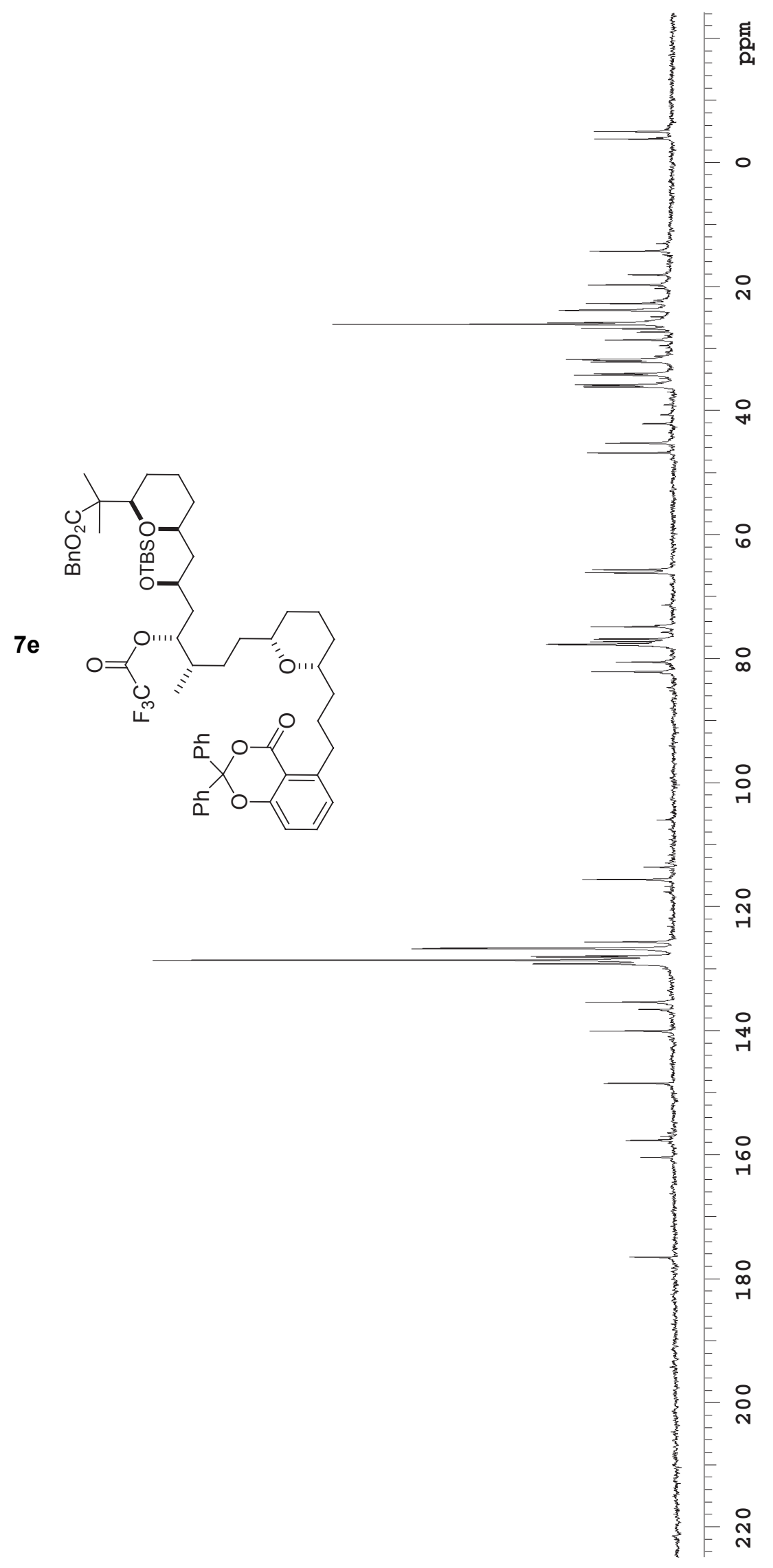




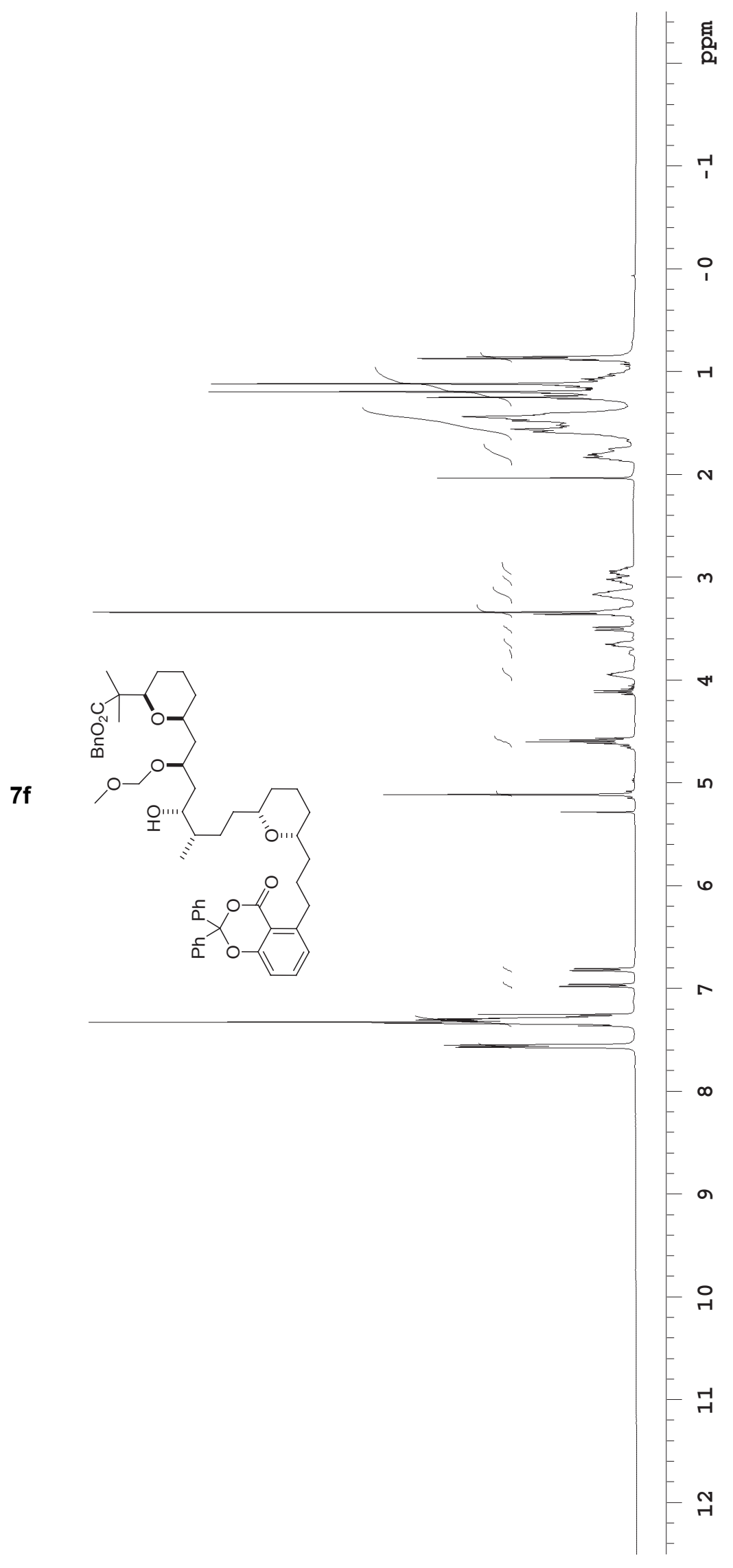




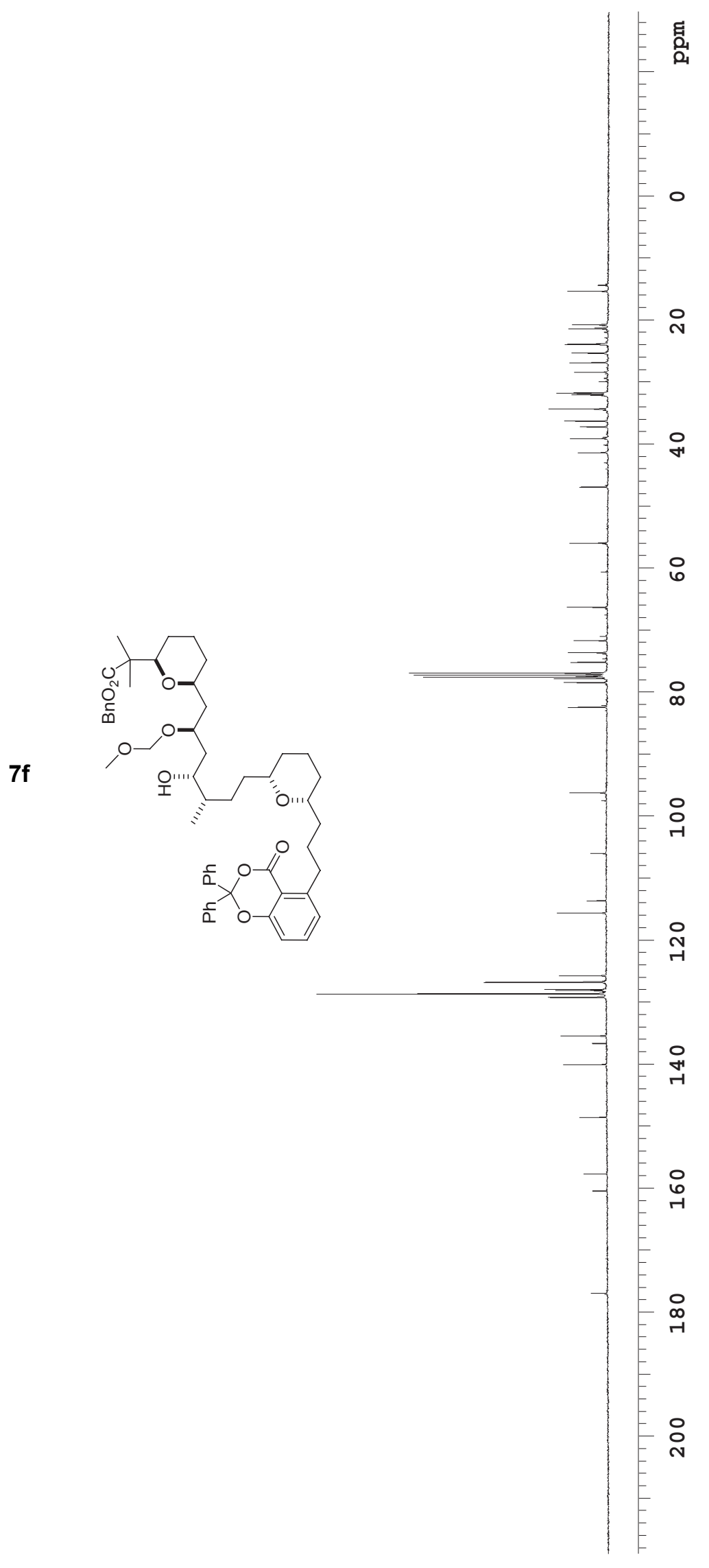




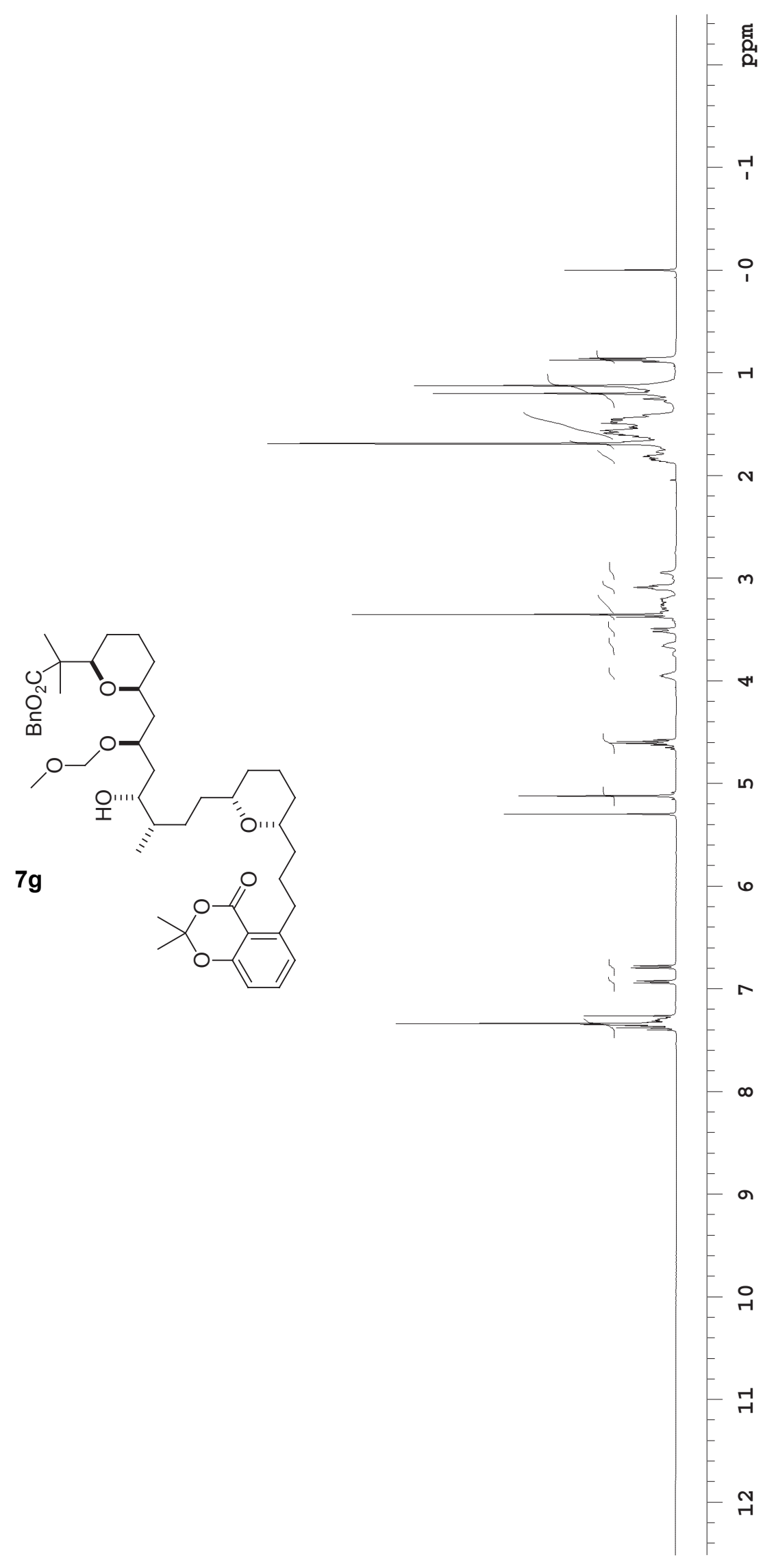




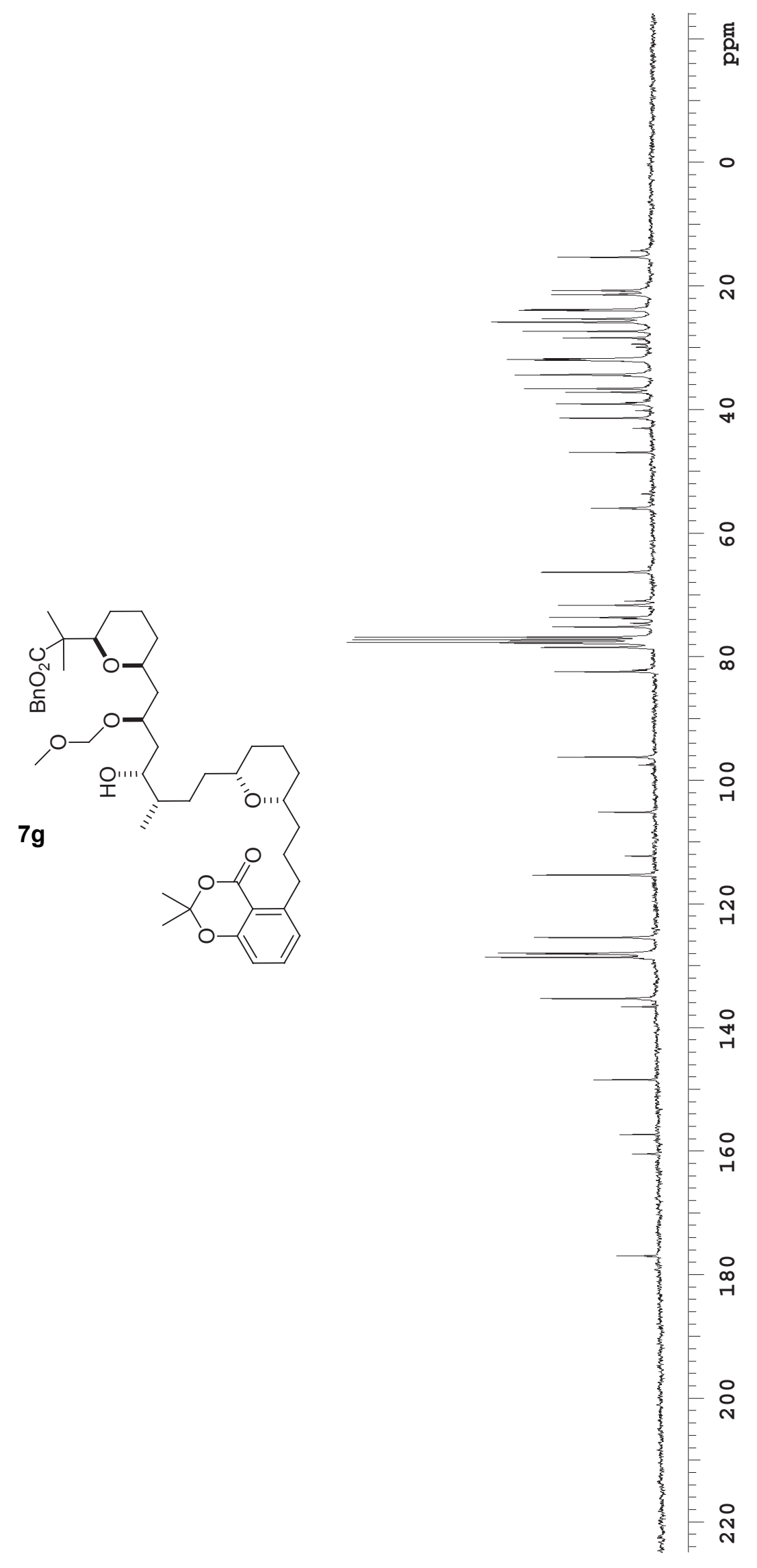




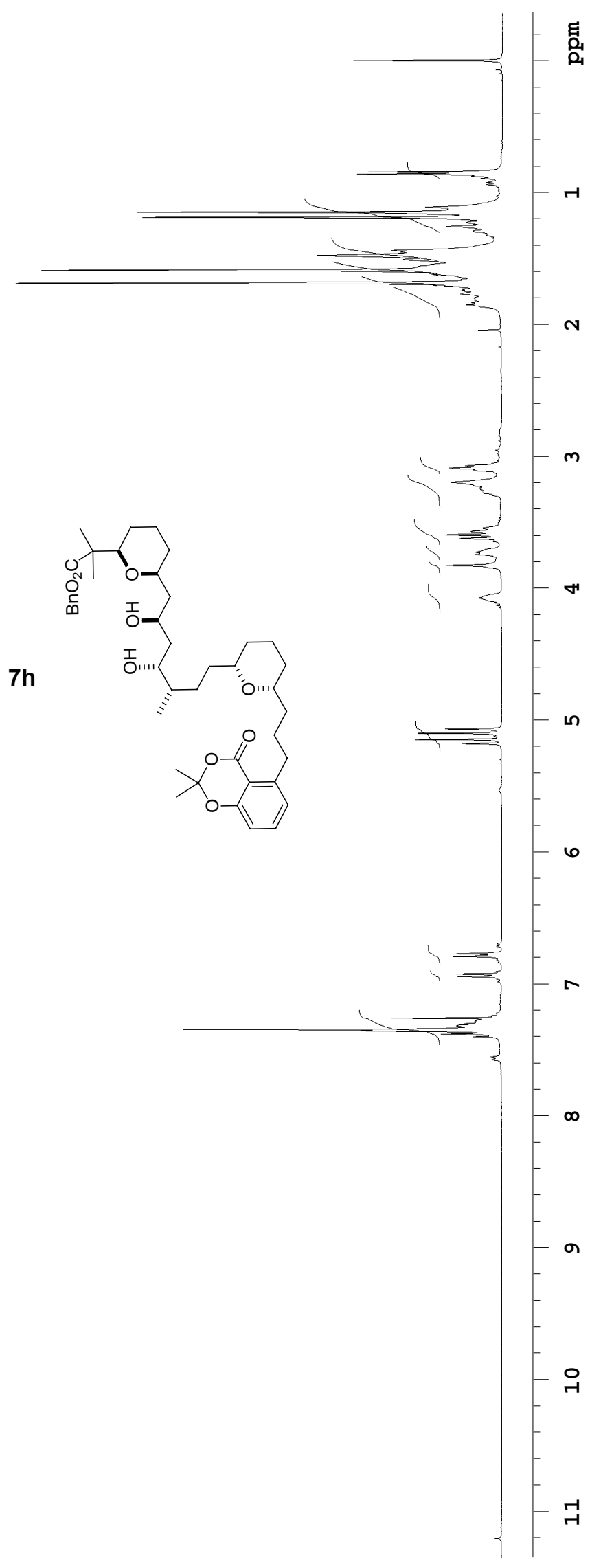




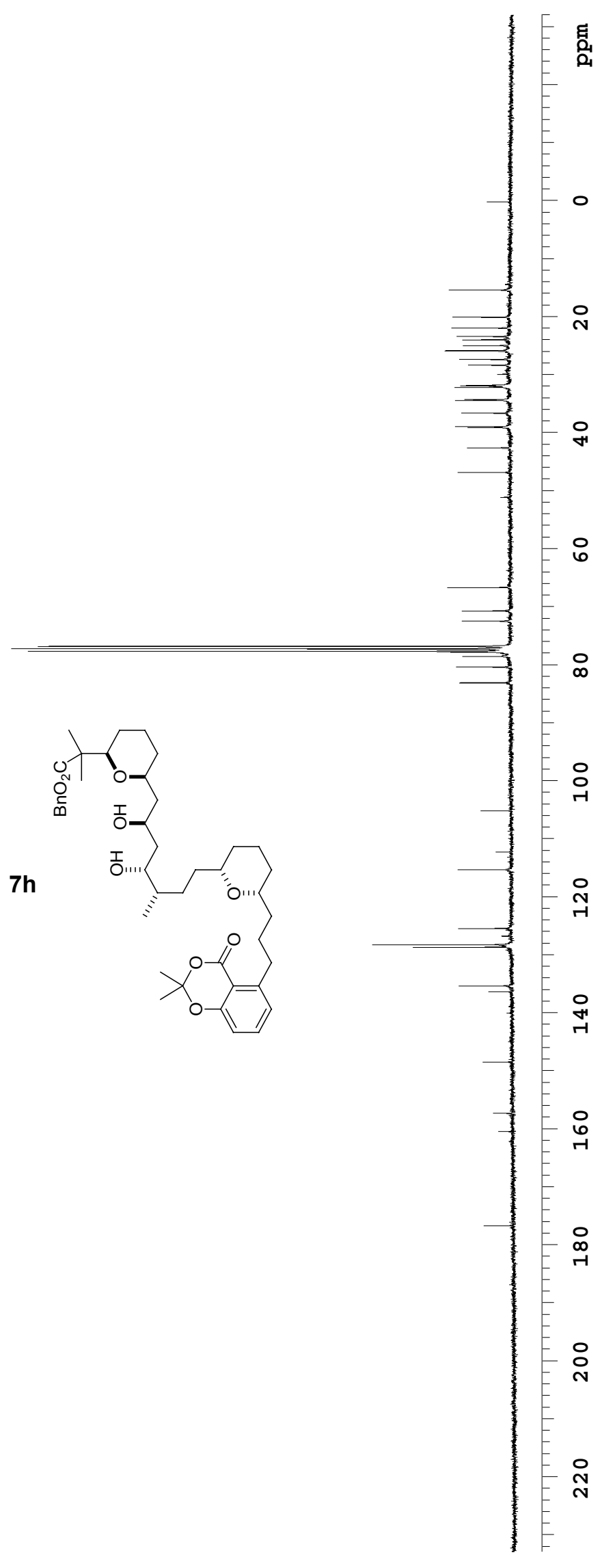




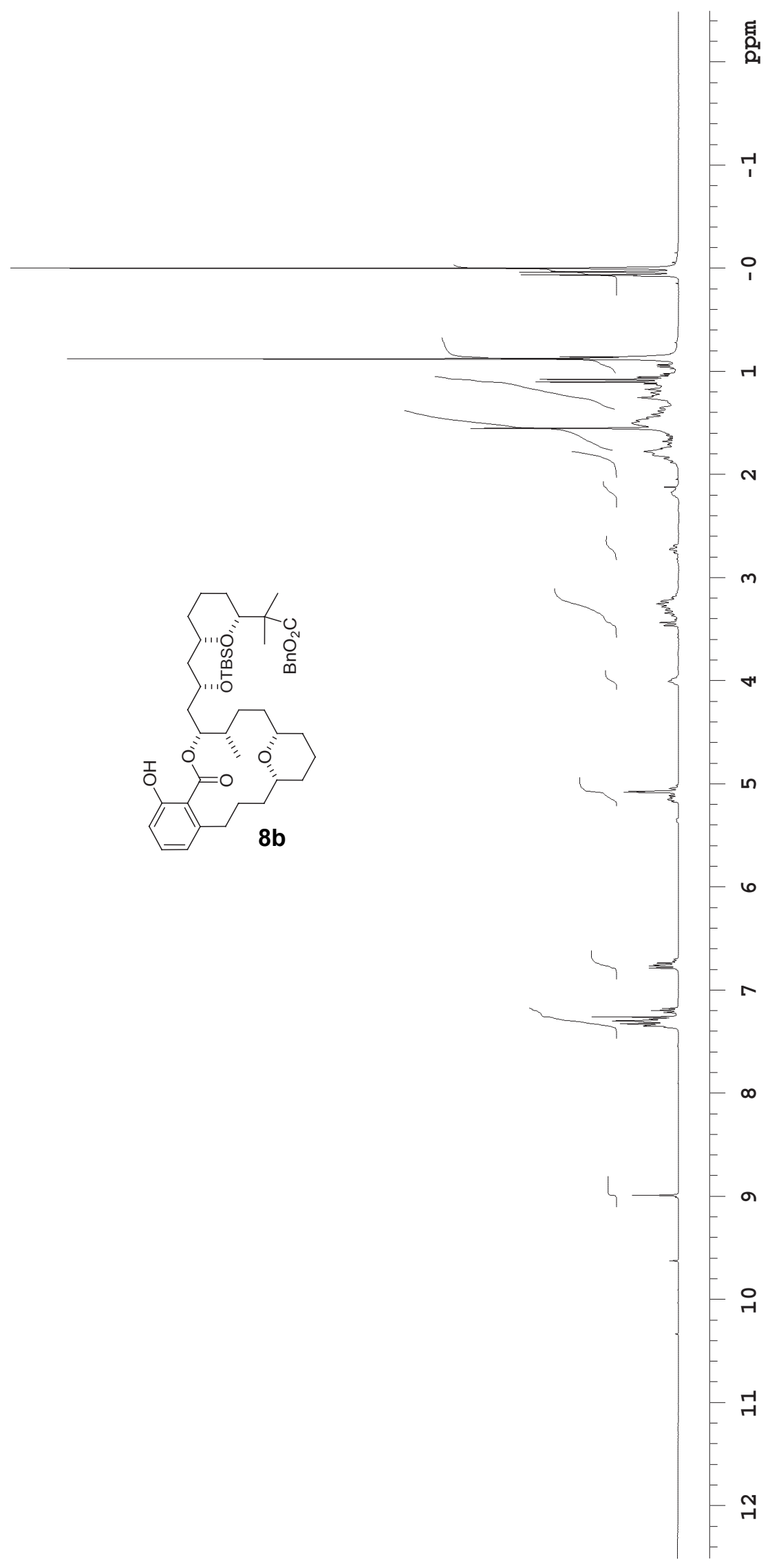




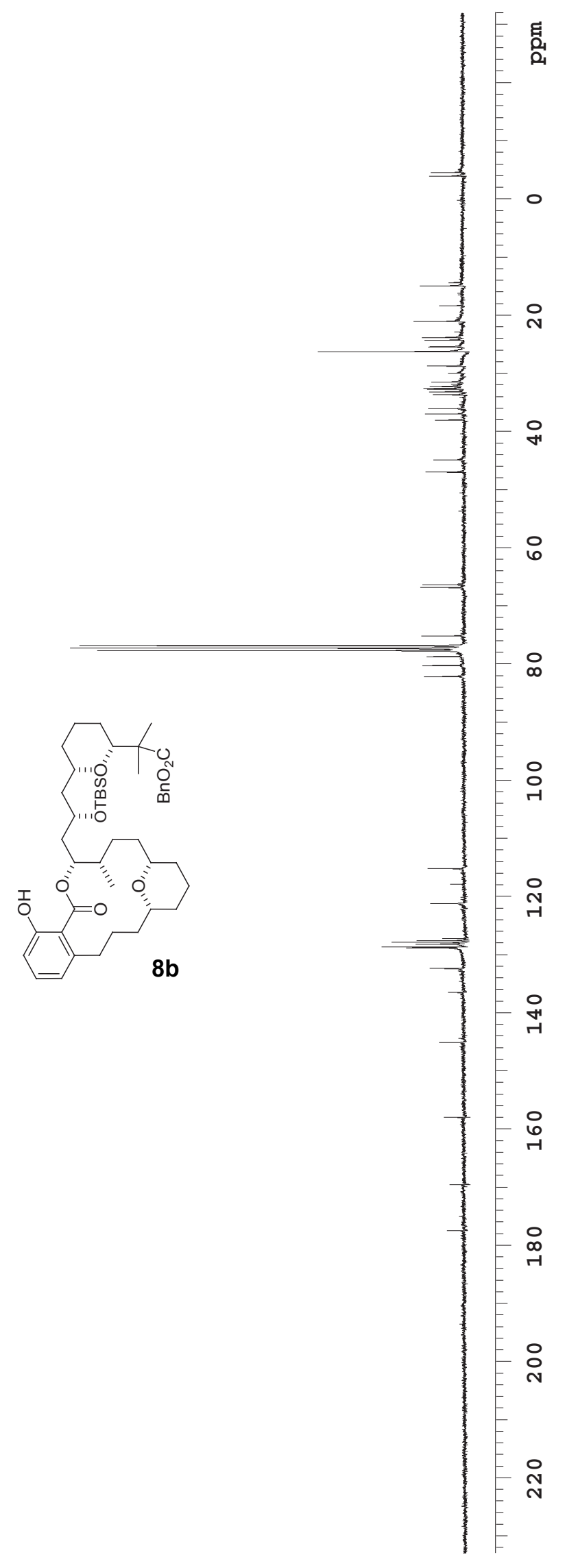




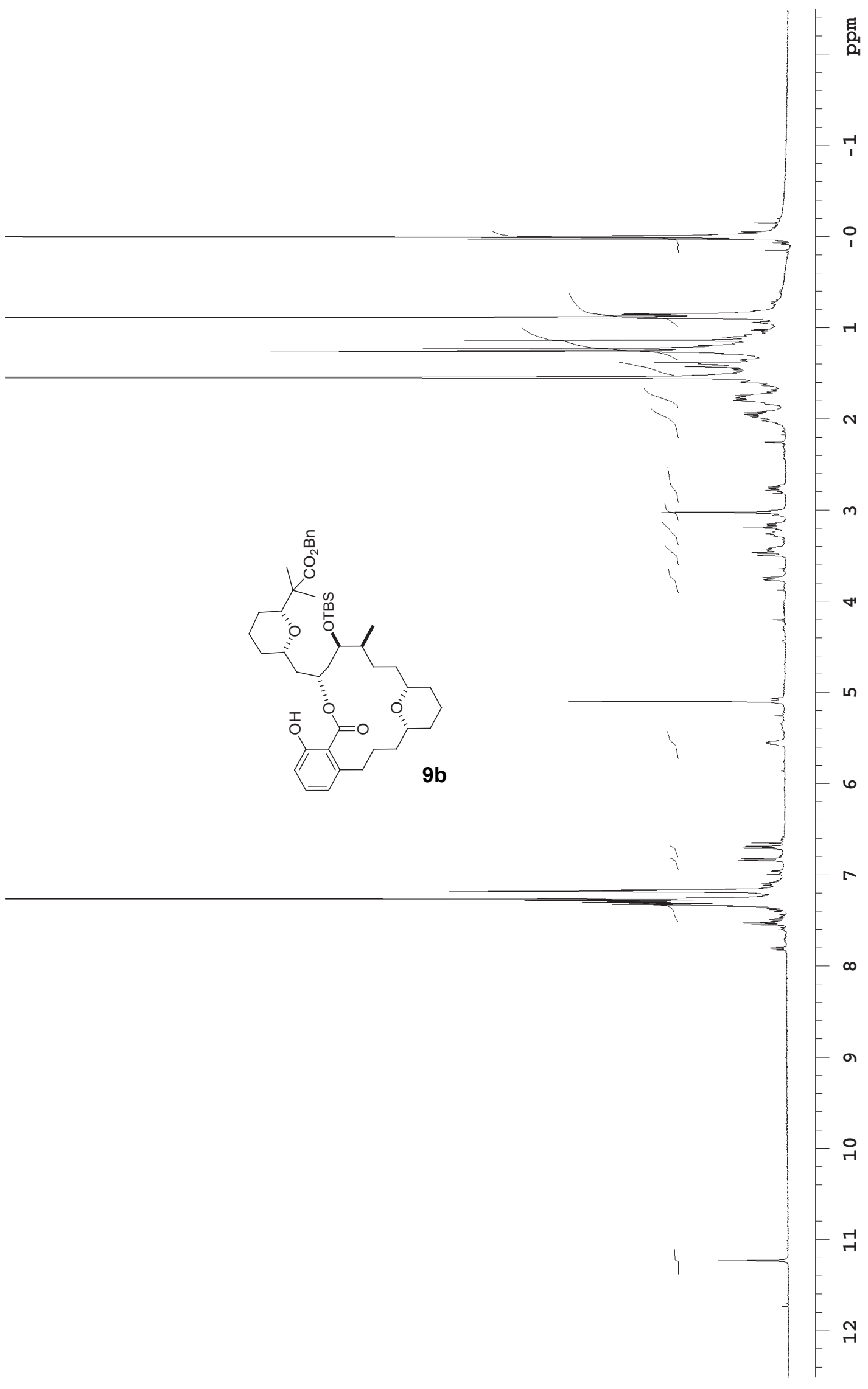




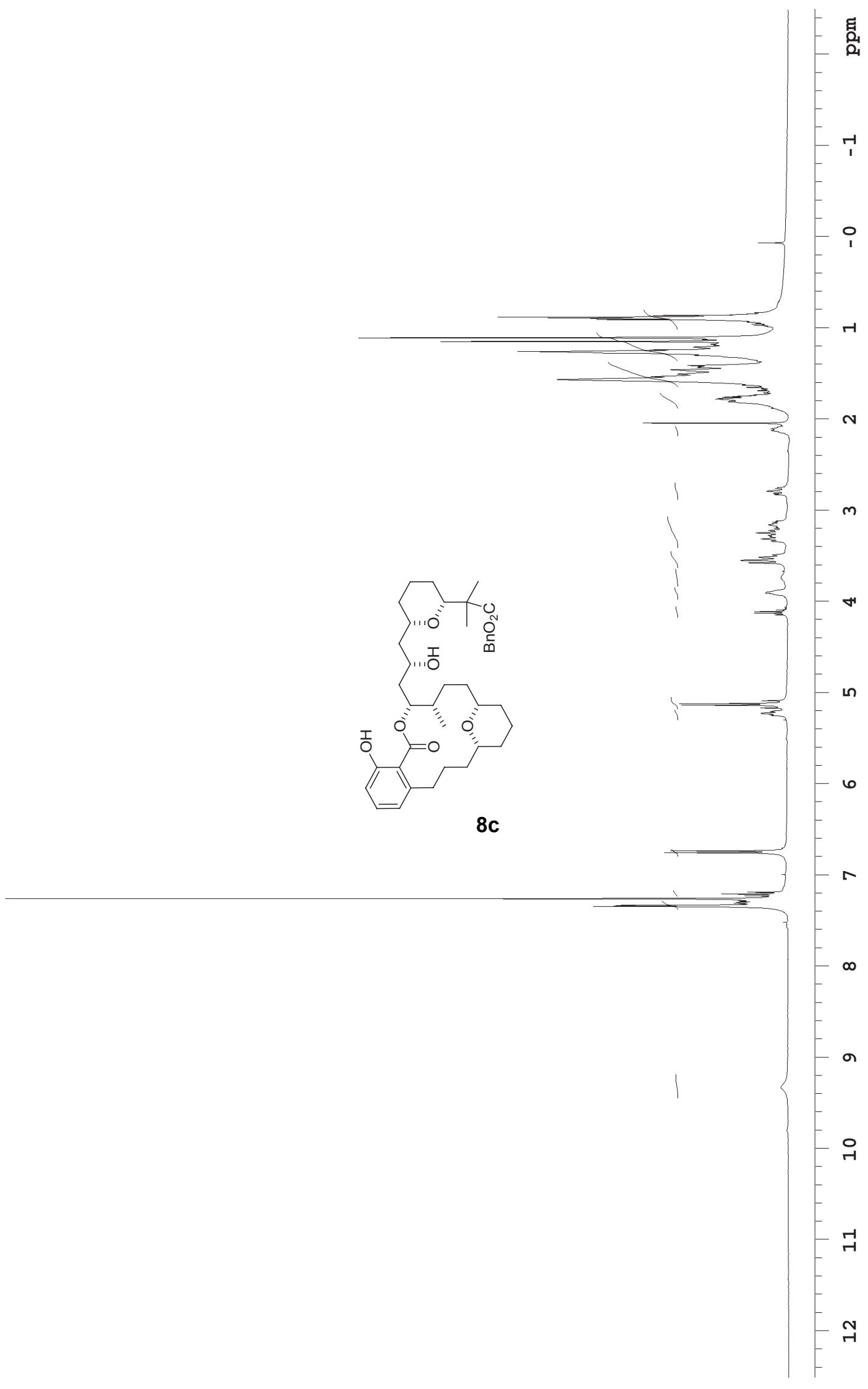




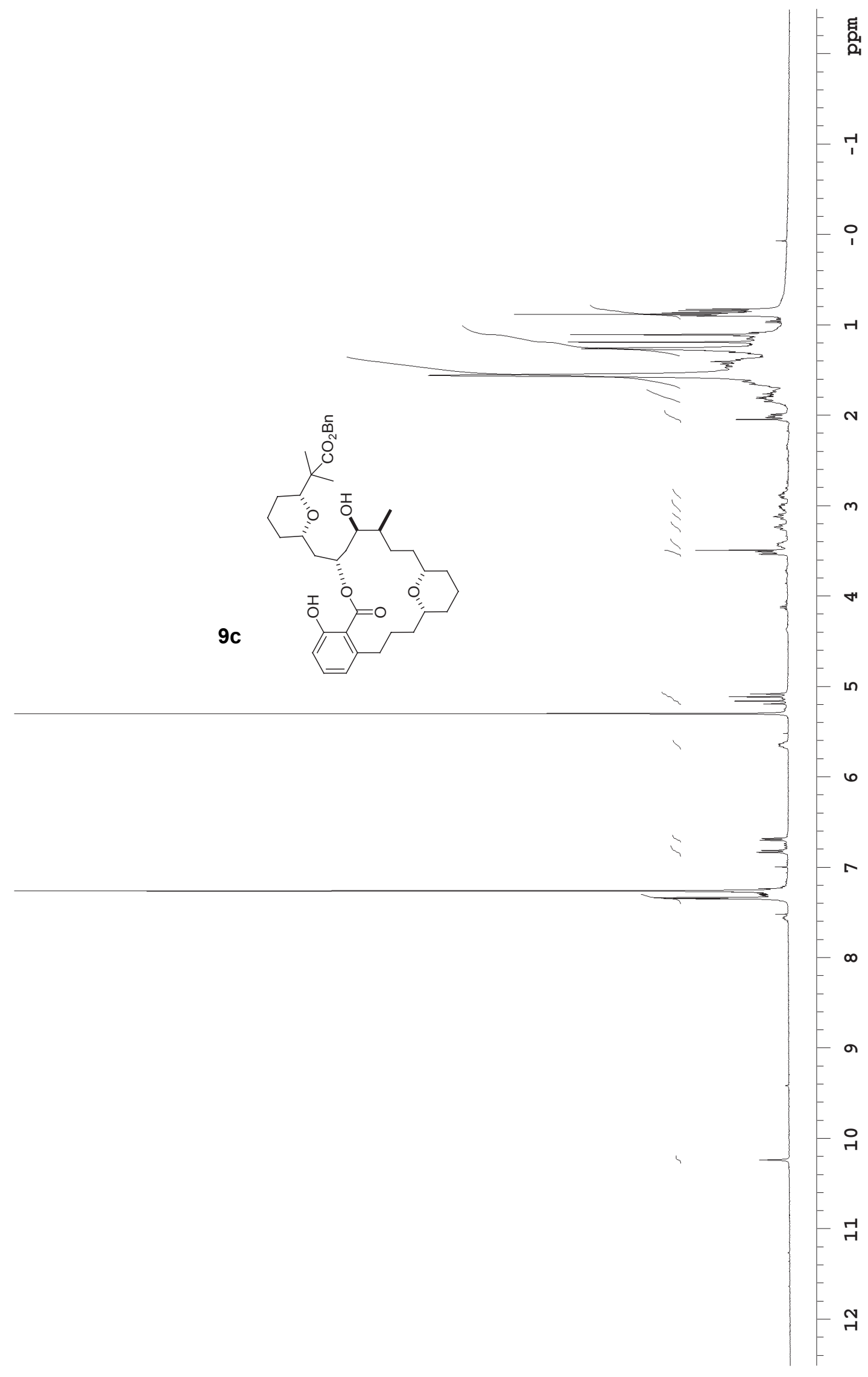




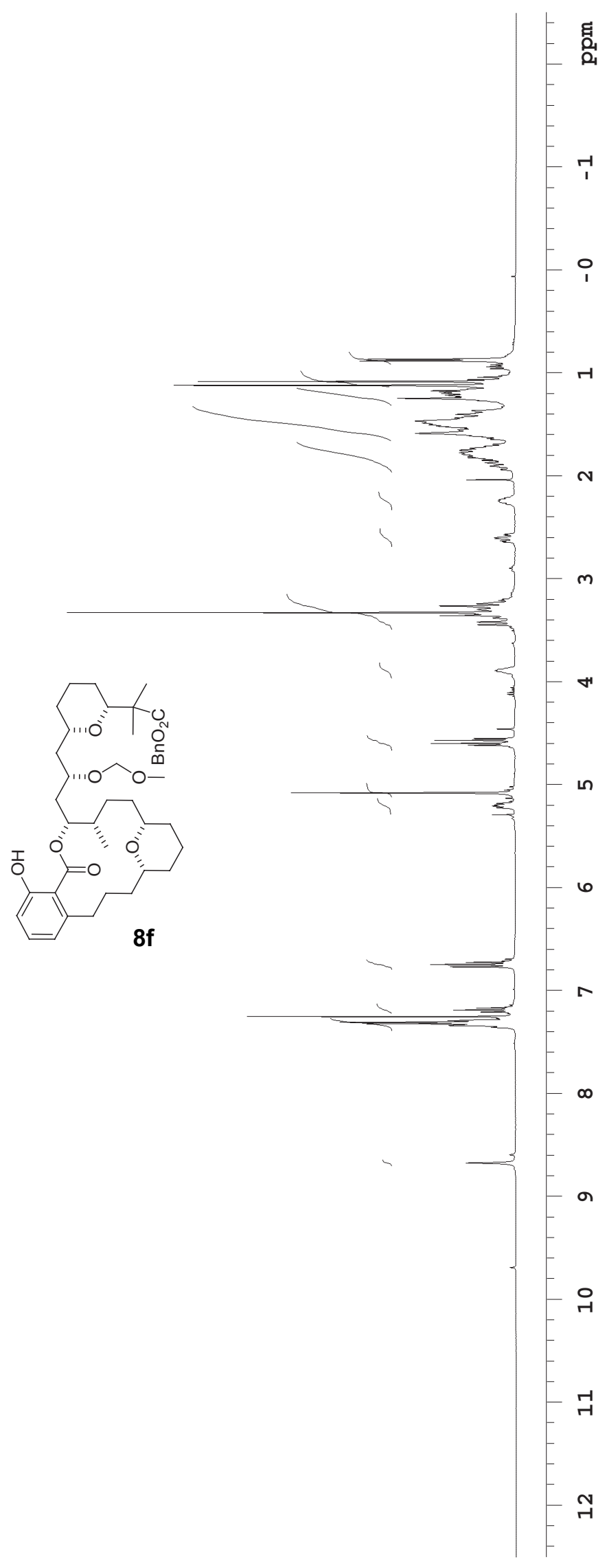




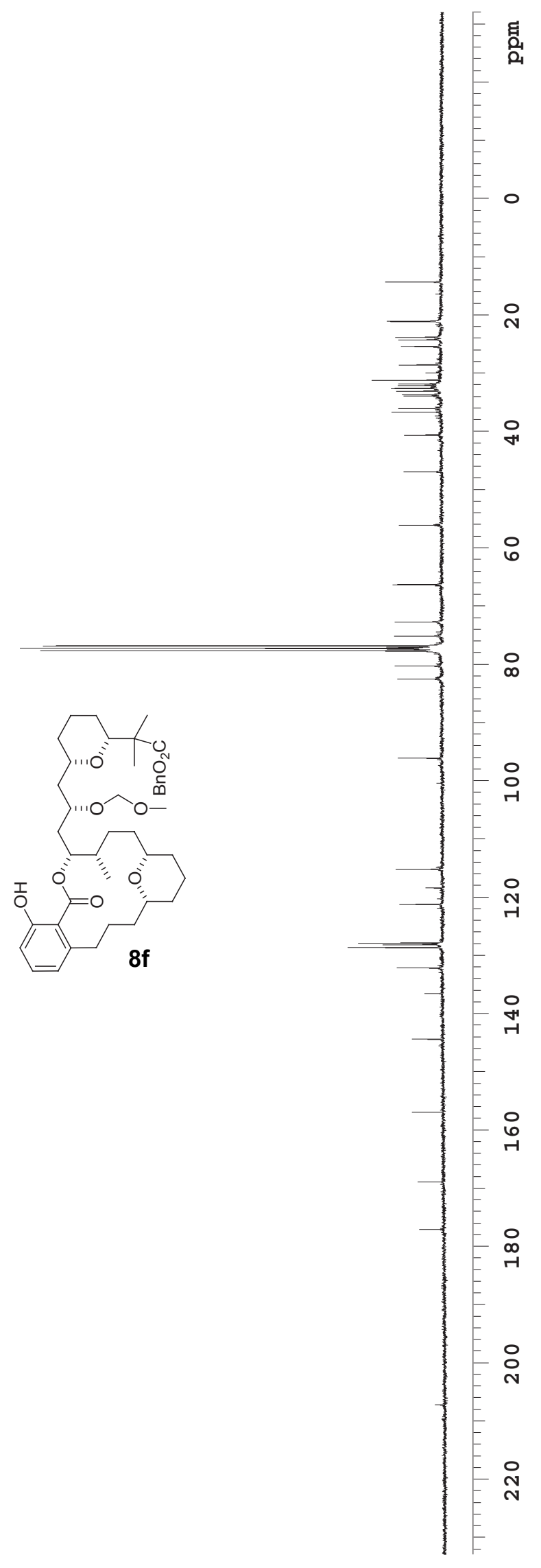




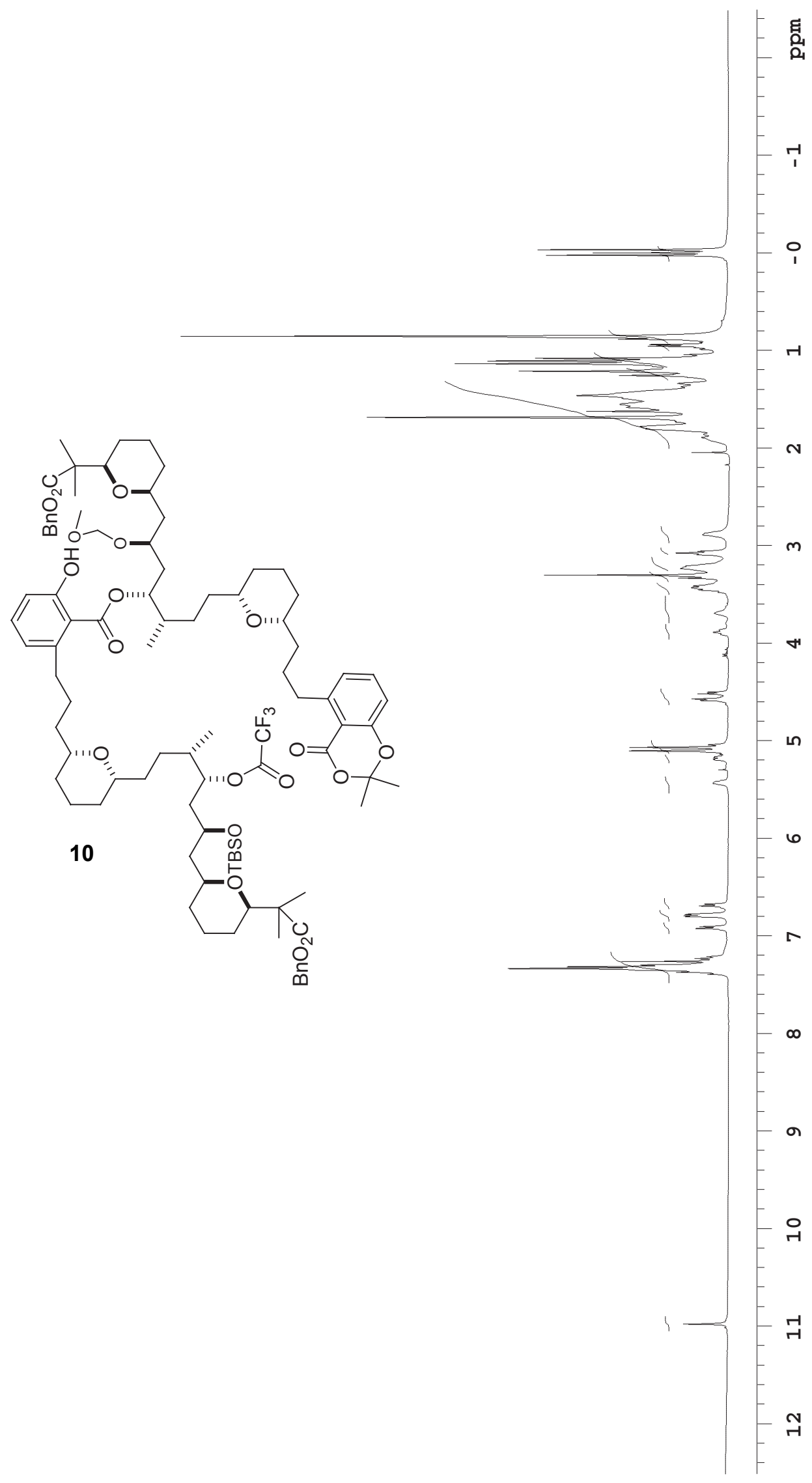




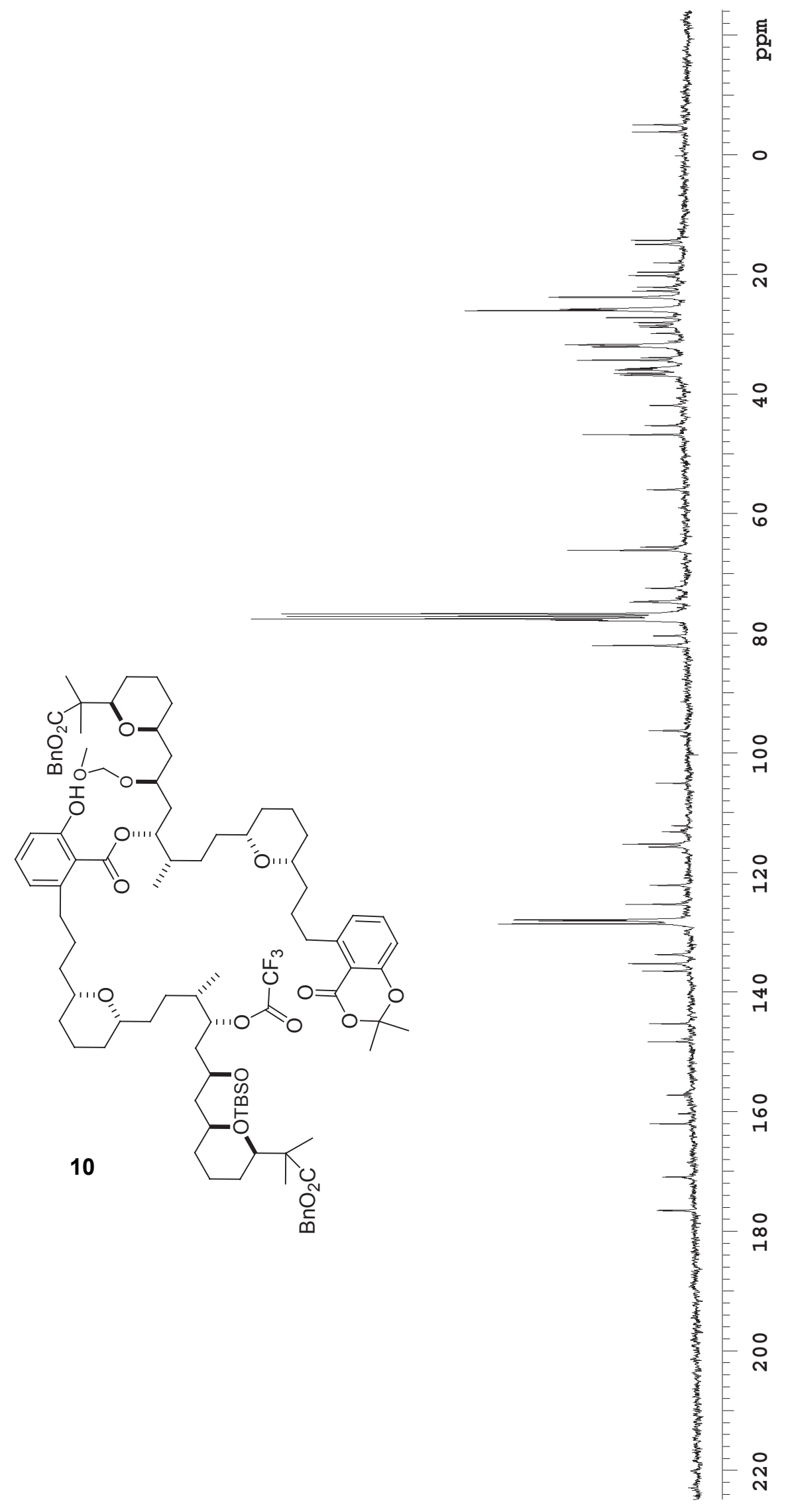




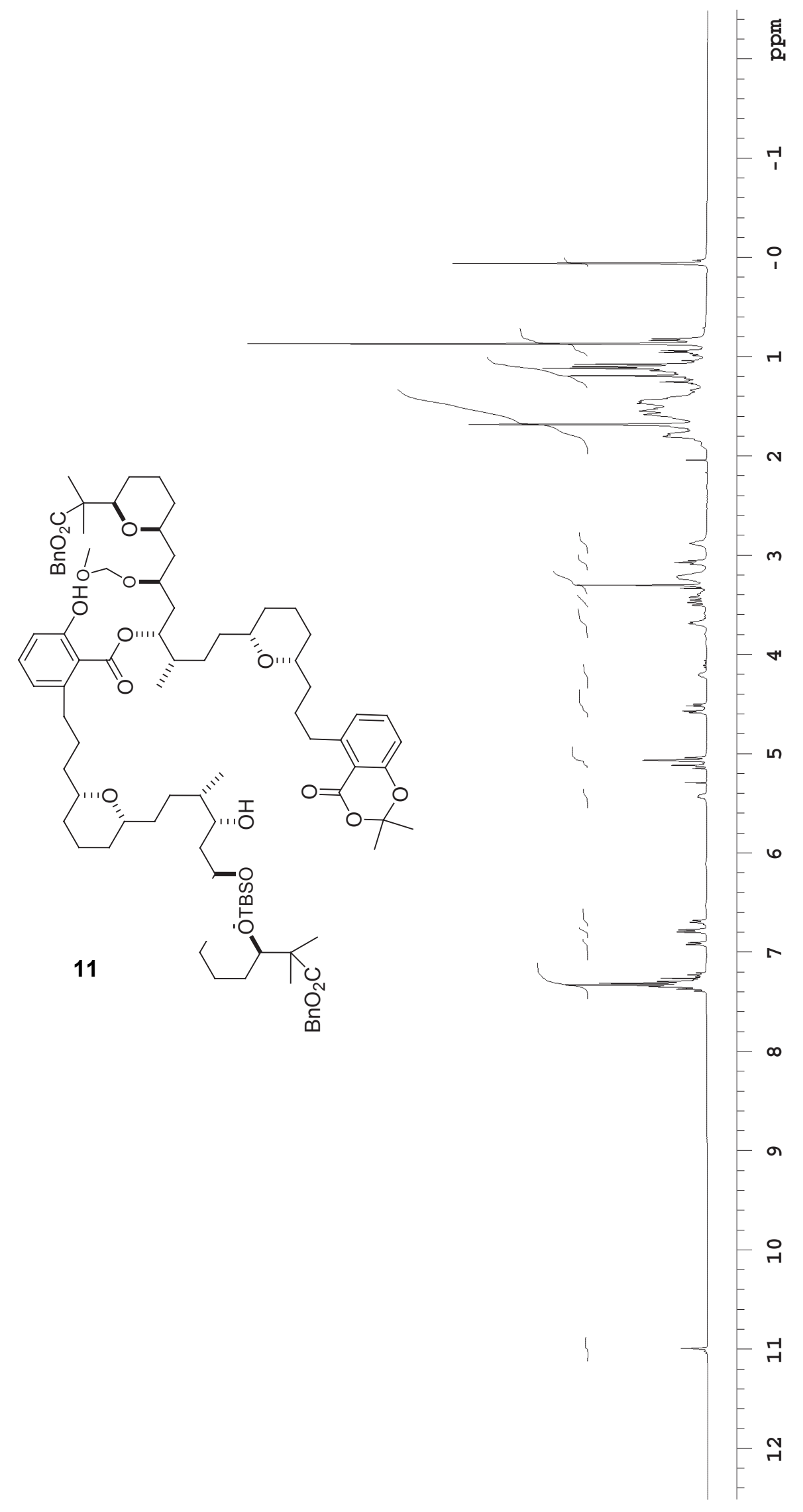




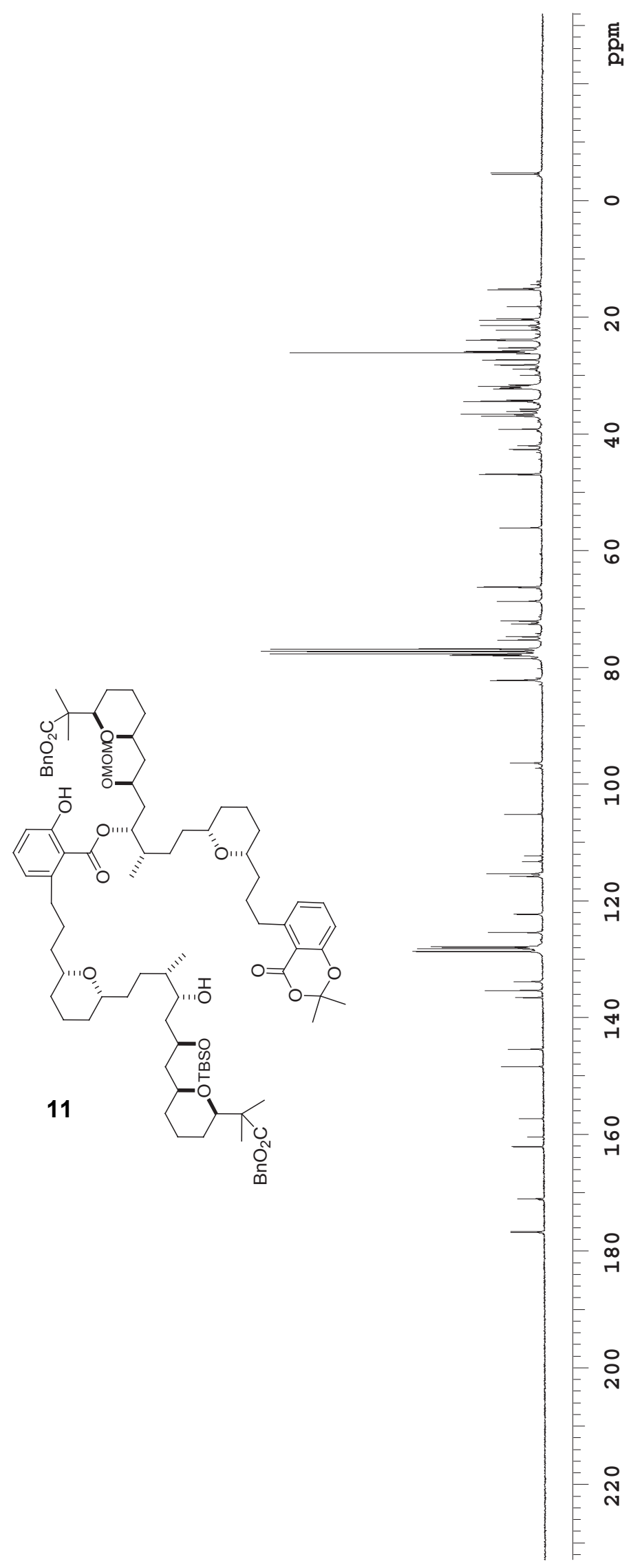




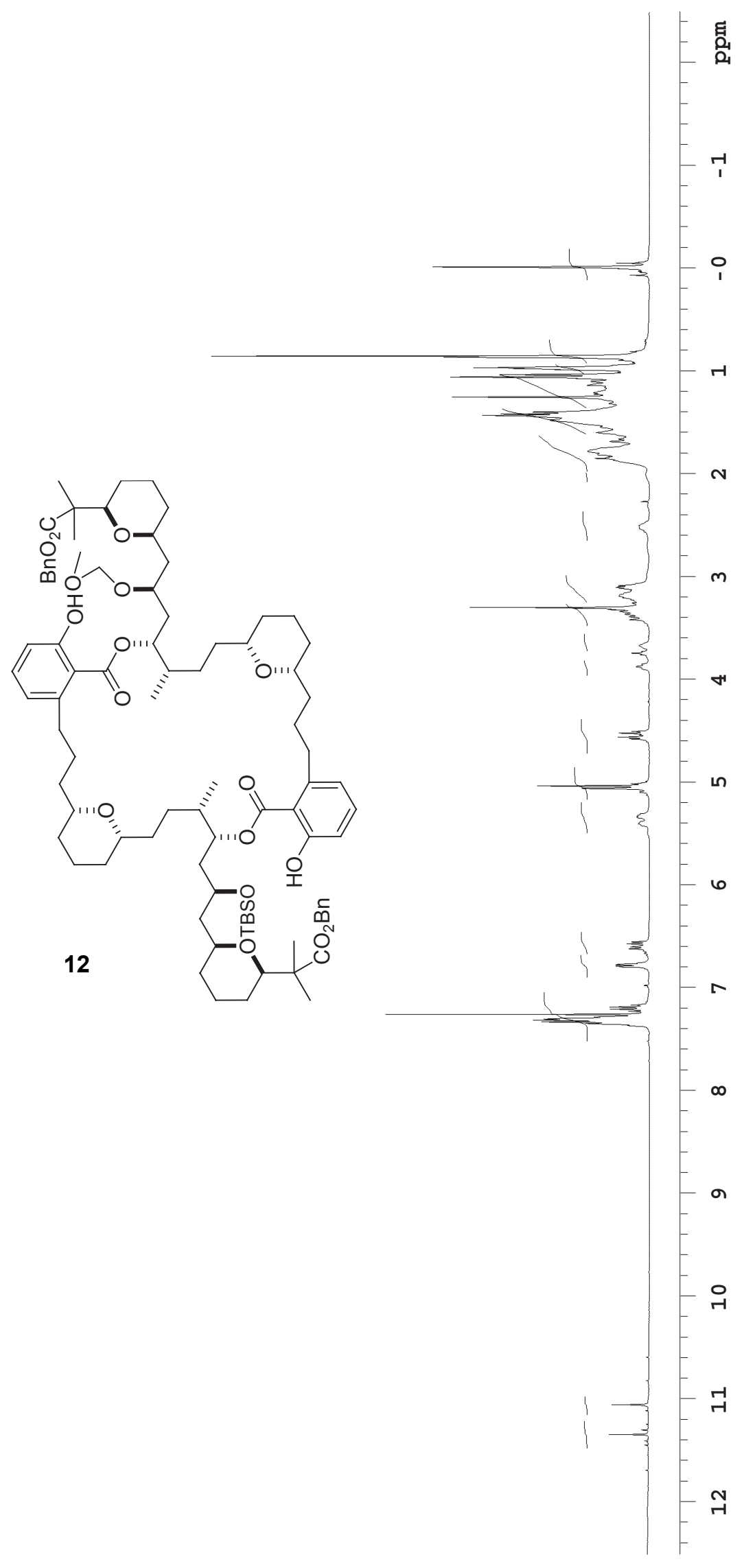




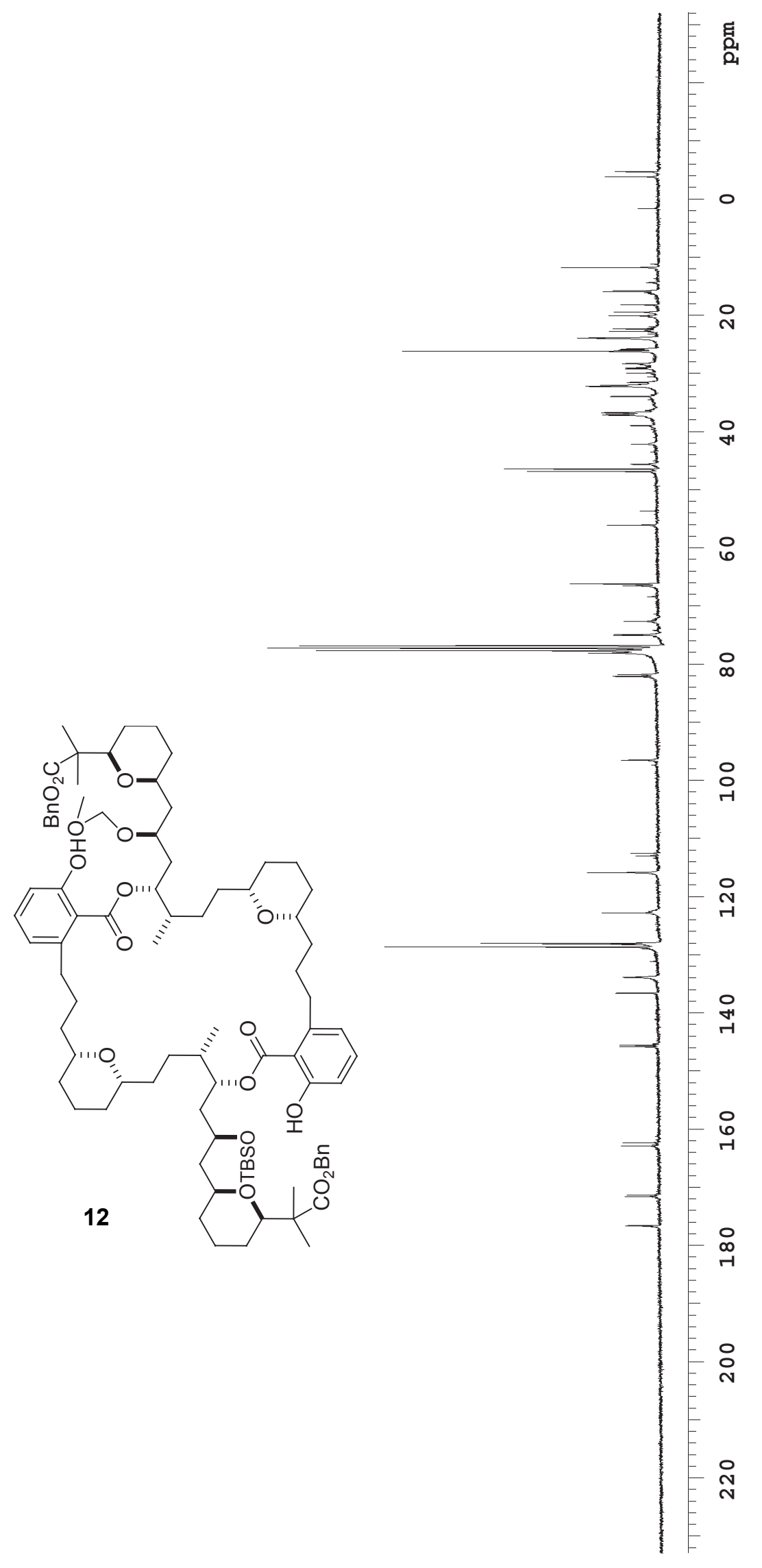




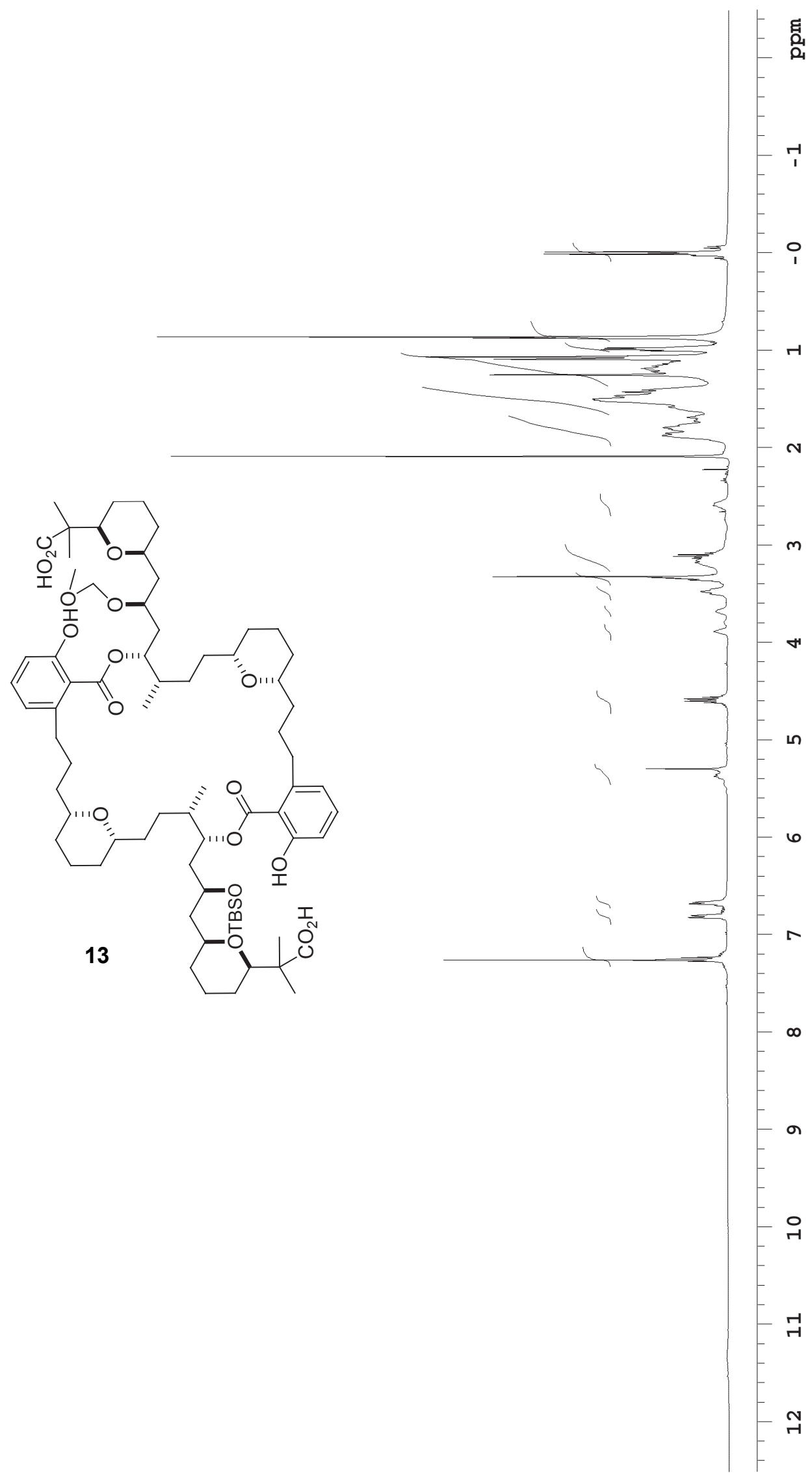




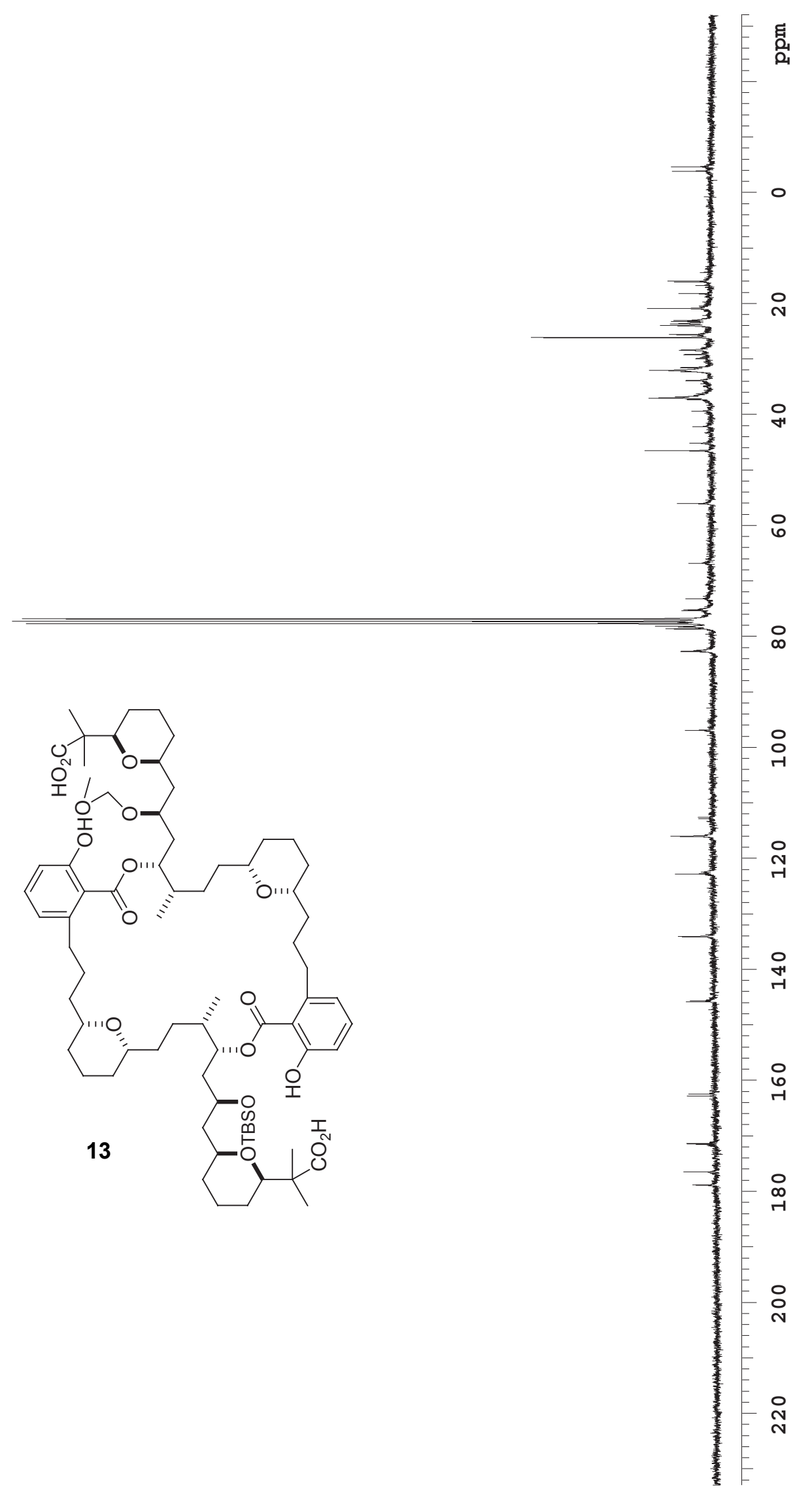



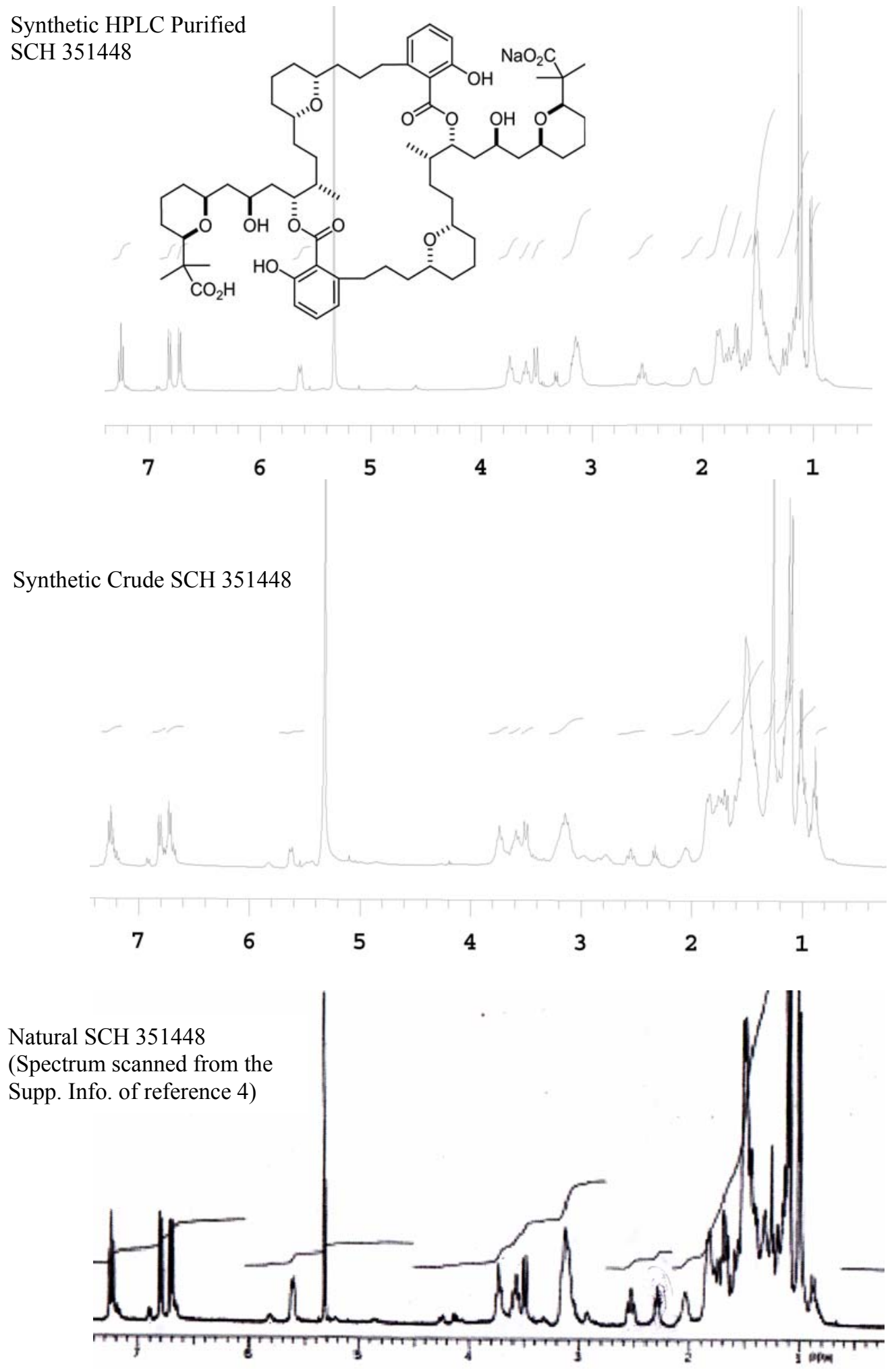

${ }^{4}$ Kang, E. J.; Cho, E. J.; Lee, Y. E.; Ji, M. K.; Shin, D. M.; Chung, Y. K.; Lee, E. J. Am. Chem. Soc. 2004, 126, 2680-2681. 


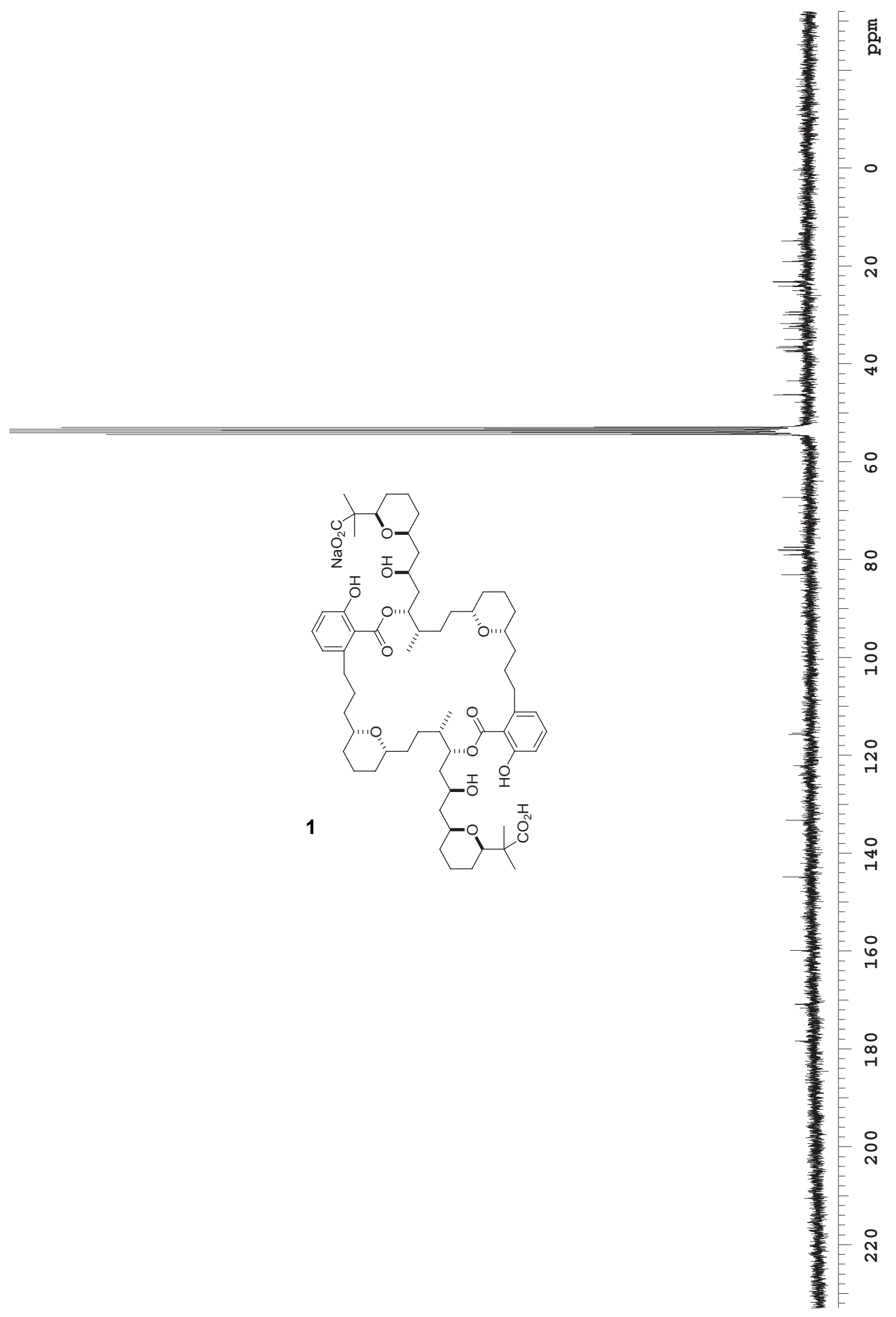

